\title{
ZUR RELATIVEN CHRONOLOGIE DER LENGYEL-KULTUR IM WESTLICHEN KARPATENBECKEN
}

\author{
PRÄLIMINARIEN ZUR BAYESISCHEN ANALYSE
}

\author{
ISTVÁN ZALAI-GAÁL* - ANETT OSZTÁS* - KRISZTINA SOMOGYI** \\ * Archäologisches Institut, Forschungszentrum für Humanwissenschaft der Ungarischen Akademie der Wissenschaften, \\ Úri u. 49, H-1014 Budapest, Ungarn \\ E-Mail: gaal.istvan@btk.mta.hu, osztas.anett@btk.mta.hu \\ ** E-Mail: lasinia1@freemail.hu
}

\begin{abstract}
The authors analyse the chronological issues of the Late Neolithic - Early Copper age at the Western part of the Carpathian Basin. The primary aim of this study is to determine the relative chronological position of Lengyel Culture graves excavated at site Alsónyék-Bátaszék, as well as to present the various ceramics types. These investigations are considered to be a preliminary study for a Bayesian analysis of radiocarbon dates, through which the absolute chronological status of the Lengyel cemetery at Alsónyék will be possible to learn in the near future.

Keywords: Late Neolithic, Eneolithic, Lengyel Culture, Western part of the Carpathian Basin, ceramics analysis, relative chronology, Bayesian analysis of radiocarbon dates
\end{abstract}

\section{EINLEITUNG}

Der Fundplatz Alsónyék-Bátaszék in Südosttransdanubien ist im Westen durch die Szekszárder Hügellandschaft und im Osten durch die breite Flussaue der ehemaligen Flussbetten der Donau begrenzt. In seiner Umgebung befinden sich inselartige Rippen und versandete Fluss- und Bachbetten 86-87 - 92-94 m über dem Meeresspiegel. Die Grabungsfläche ist in der Mitte durch das Bachbett Lajvér grob in zwei Teile geteilt. Die ursprünglichen oberflächenmorphologischen Gebilde des Bereiches wurden vor allem durch Wasserschutzmaßnahmen bis zum Ende des 19. Jahrhunderts völlig verwandelt.

Die Fundstelle erstreckt sich am Grenzgebiet zweier Landschaften verschiedenen Typs, nämlich der Transdanubischen Hügellandschaft und der Großen Ungarischen Tiefebene. Sie liegt in der Nähe der Donau, die auch in den prähistorischen Zeiten als Hauptverkehrsstraße gedient hatte. Im Fall dieses geographischen Gebietes handelt es sich sonst um die Kontaktzone der neolithischen Populationen des nördlichen Grenzgebietes des Balkans und Mitteleuropas. Diese geographische Situation ergab auch den Abdruck der in der materiellen Kultur fassbaren südlichen kulturellen Wirkungen in allen neusteinzeitlichen Zeitperioden (Abb. 1). ${ }^{1}$

Die archäologischen Rettungsgrabungen wurden vor den Bauarbeiten der Autobahn M6 in Südtransdanubien zwischen 2006 und 2009 durchgeführt. Die verschiedenen Grabungsareale bilden die Teile einer einzigen großen neolithischen Fundstelle, an der eine Fläche von insgesamt $250000 \mathrm{~m}^{2}$ und etwa 15000 archäologische „Erscheinungen“ untersucht wurden. Etwa $90 \%$ der ausgegrabenen und dokumentierten Befunde stammt aus den

${ }^{1}$ Fotos: F. Fazekas; Zeichnungen: S. Ősi und F. Tortoriello (Forschungszentrum für Humanwissenschaften der Ungarischen Akademie der Wissenschaften, Archäologisches Institut, Budapest). 


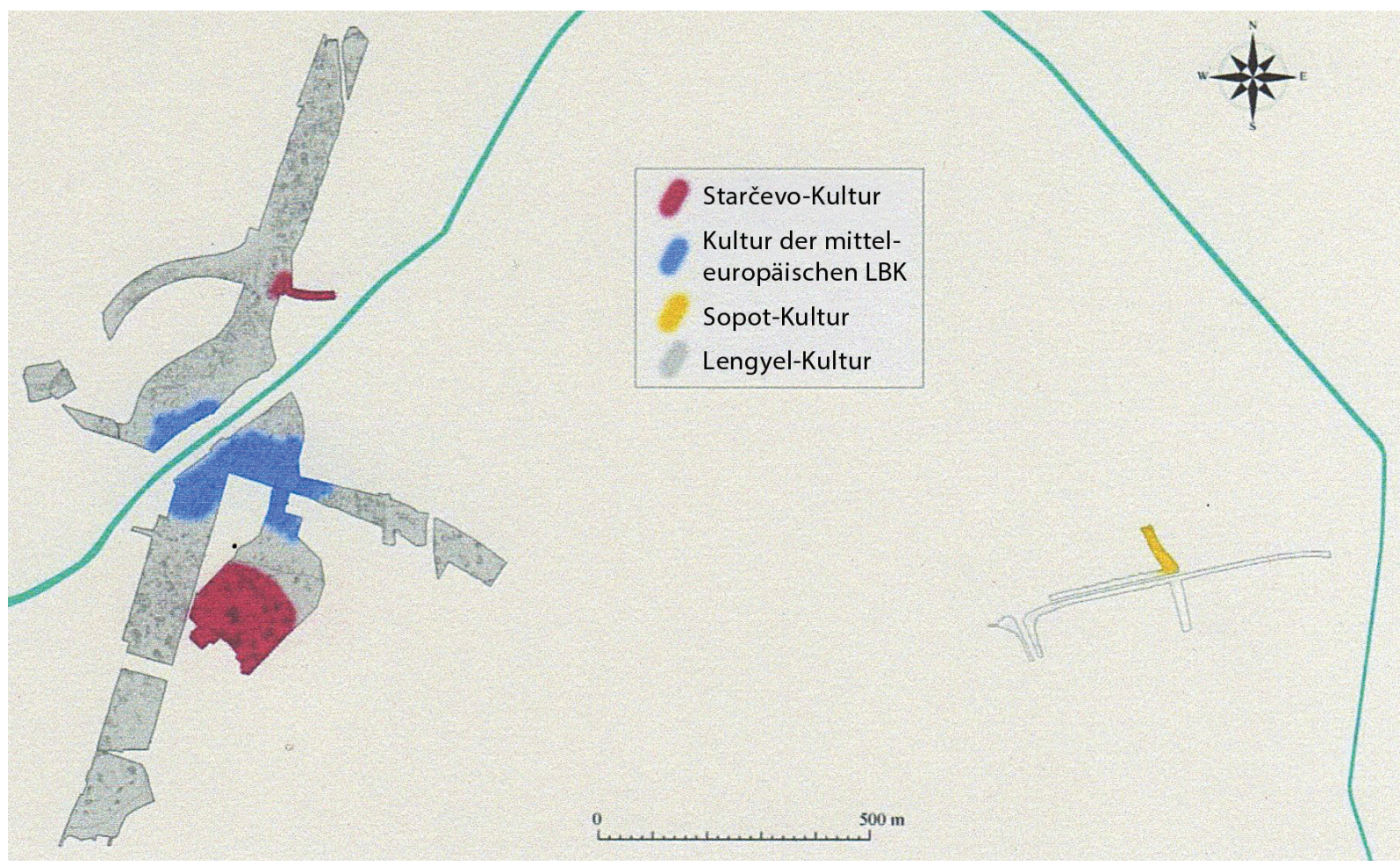

Abb. 1. Untersuchte Grabungsflächen der neolithischen Kulturen von Alsónyék-Bátaszék

Zeitperioden des Neolithikums. Die spätneolithische Siedlung erstreckt sich in Nord-Süd-Richtung nahezu 1,5 km und in West-Ost-Richtung $800 \mathrm{~m}$ lang. Ihre geschätzte Größe beträgt ca. 80 Hektar.

Der Fundplatz erreichte seine größte Ausdehnung während des Spätneolithikums. Außer Tausenden von ausgegrabenen Gruben wird das auch durch 2359 Bestattungen $^{2}$ und Reste von nicht weniger als 123 Häusern mit Pfostenkonstruktion bewiesen.

Die Fundgeschichte und die einzelnen Aspekte der Funde und Befunde dieses Fundplatzes wurden bislang in mehreren Studien und Vorberichten behandelt. ${ }^{3}$

\subsection{Zielsetzung}

In der vorliegenden Arbeit versuchen wir die relativchronologische Stellung des Gräberfeldes von Alsónyék-Bátaszék (im Weiteren: Alsónyék) innerhalb des weiten Verbreitungsgebietes der Lengyel-Kultur im westlichen Karpatenbecken mit Hilfe der Merkmalanalyse und Seriation der Grabkeramik festzustellen. Dazu nahmen wir insgesamt 1057 Grabgefäße oder Bruchstücke von solchen auf, die in 439 Bestattungen von 13 Nekropolen (Fundorten) der Lengyel-Kultur hingelegt wurden. Von der Fundstelle Alsónyék 10B konnten wir 91 Gefäße aus 42 Bestattungen analysieren, während die Zahl der untersuchten Keramikgegenstände aus 35 Gräbern der Fundstelle 5603 nicht mehr als 106 beträgt.

Als der erste Schritt werden die charakteristischen Typengruppen und Formvarianten (Typen) aus den Fundstellen 10B und 5603 von Alsónyék vorgestellt und es wird auf ihre typologischen Analogien im Lengyel-Kreis hingedeutet. Als der zweite Schritt versuchen wir die relativchronologische Entwicklung (Belegungsgeschichte) der

${ }^{2}$ Die symbolischen Bestattungen werden hier nicht gezählt.
${ }^{3}$ ZALAI-GAÁL 2008a; ZALAI-GAÁL-OSZTÁs 2009a; 2009b; ZALAI-GAÁL-KÖHLER-OsZTÁs 2010; KÖHLER et al. 2012; OsZTÁs et al. 2012; SOMOGYI-GALlina 2013; GALlina et al. 2010; GelencSÉr 2010. 
diskutierten lengyelzeitlichen Gräberfelder, erstens anhand der Analyse der Typengruppen, zweitens auf Grund der Formvarianten, festzustellen.

Unsere Ergebnisse sollen bezüglich der Relativchronologie der lengyelzeitlichen Gräberfelder im westlichen Karpatenbecken dann im Rahmen der Bayes'schen Forschungen, im Verhältnis von AMS-Daten ausgewertet werden: „Da insgesamt noch viel zu selten Radiokarbondaten gemessen wurden, sind die Geschwindigkeiten des Stilwandels in den einzelnen Regionen noch nicht zu entschlüsseln. Eine exakte überregionale vergleichende Periodisierung insbesondere der ersten Hälfte des 5. Jahrtausends scheint aus diesem Grunde nicht möglich“ - bestätigt R. Gleser. ${ }^{4}$

\subsection{Forschungsgeschichtlicher Überblick}

Im Zusammenhang mit den wichtigsten Fragen der relativen Chronologie der Lengyel-Kultur können wir hier Folgendes resümieren.

A. Točík, J. Lichardus und S. Šiška stellten für die mit bemalter Keramik charakterisierten Entwicklung der Lengyel-Kultur in der Südwestslowakei drei Stufen - Lengyel I, Lengyel II und Lengyel III - fest. ${ }^{5}$ Die Stufe Lengyel III wird dabei in erster Linie durch pastose weiße Gefäßbemalung gekennzeichnet, während die Gefäßbemalung in der Stufe Lengyel IV verschwindet. Die Stufe Lengyel V hat nur unverzierte Keramik und ist typologisch eine Fortsetzung der Stufe Lengyel IV. ${ }^{6}$ Das letzte Chronologiesystem von J. Lichardus stammt aus 2003.

Das chronologische System von J. Pavúk umfasst dagegen vier Phasen, für die mit bemalter Keramik bezeichnete Entwicklung bestimmte er nämlich nur zwei Stufen (Lengyel I und Lengyel II). ${ }^{8}$

Im Rahmen seiner Arbeit hat M. Doneus die bereits seit Längerem existierende Periodisierungen der mährisch-bemalten Keramik (MBK) auch für die österreichische Verbreitung , und somit für die gesamte mährischostösterreichische Gruppe der Lengyel-Kultur erweitert" ${ }^{9}$ Die Forschungen von P. Stadler und E. Ruttkay haben die absolutchronologischen Verhältnisse der österreichischen Lengyel-Phasen mit Hilfe von AMS-Daten geklärt. ${ }^{10}$

Die erste ungarische Lengyel-Chronologie von N. Kalicz stimmt im Wesentlichen mit deren von I. BognárKutzián überein. ${ }^{11}$ Die ritzverzierte und rot bemalte Keramik wurde, zusammen mit den Funden aus Lužianky, in die Frühstufe eingestuft („,klassische“ Fundorte der Lengyel-Kultur in Südtransdanubien und Aszód). ${ }^{12}$ In die spätere Stufe wurden die Funde aus Somodorpuszta, Zalavár, Lébény und Szob eingereiht und N. Kalicz datierte auch einen Teil der Gräber von Zengővárkony in die spätere Stufe. ${ }^{13}$ Anhand der Funde von Lánycsók folgerte er darauf, dass sie einen Übergang zwischen den älteren und jüngeren Lengyel-Stufen bilden. ${ }^{14}$

Die Befunde und Funde der mit unbemalter Keramik bezeichneten Entwicklungsstufe der Lengyel-Kultur veröffentlichte P. Raczky vom nordtransdanubischen Fundort Veszprém-Felszabadulás út: Diese Keramikgegenstände entsprechen typochronologisch denen des südwestslowakischen Nyitra-Brodzány-Horizontes. ${ }^{15}$ In Zusammenhang mit dem Vorkommen der Stufe Lengyel III in Nordtransdanubien sind auch die Forschungen von J. Regenye zu erwähnen. ${ }^{16}$ Diese mit unbemalter Keramik gekennzeichnete Zeitperiode der Lengyel-Kultur wurde in Südtransdanubien bislang noch nicht nachgewiesen. Aus Sicht der Wirkungen der Lengyel-Kultur im Spätneolithikum der Großen Ungarischen Tiefebene brachten die Forschungen von K. Sebők wichtige Ergebnisse. ${ }^{17}$ Nach den möglichen Wirkungen und Gegenwirkungen zwischen dem westlichen und östlichen Karpatenbecken suchten

\footnotetext{
${ }^{4}$ GLESER 2012, 82.

5 ToČíK-Lichardus 1966, 87; Lichardus-ŠIŠKA 1970 , $342-344$.

${ }^{6}$ LiCHARDUS 1974, 34-35.

${ }^{7}$ Lichardus-Vladár 2003, 203-204: Protolengyel (Lužianky, Grabfunde); Lengyel I (Grabfunde aus Svodín, Siedlungsfunde); Lengyel II (Grabfunde aus Svodín, weitere Siedlungsfunde); Lengyel III (Grab- und Siedlungsfunde aus Pečeňady, Vel'ké Kostolany); Topolcsány-Szob-Horizont (Grabfunde aus Topolcsány); Lengyel IV (Nyitra-Brodzány-Stufe); Lengyel V (Ludanice-Gruppe).

${ }^{8}$ PAvÚK 1981: Protolengyel 1 = Bíňa-Bicske; Protolengyel 2 = Lužianky; Lengyel I = Svodín; Lengyel II (Vel'ké Kostolany und Pečeňady), Moravany-Übergangsphase; Lengyel III und Lengyel IV.
}

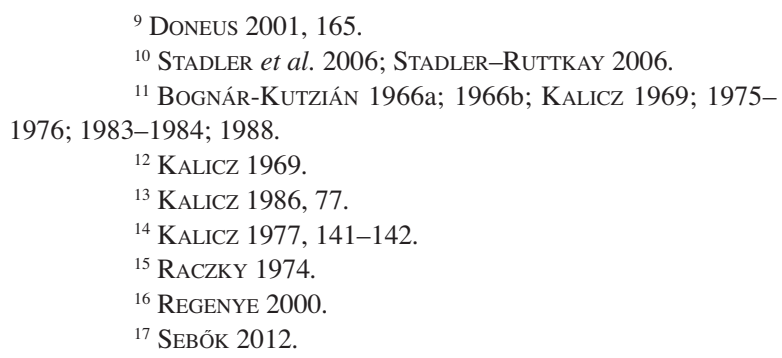


wir auf Grund der Merkmalanalyse und Seriation der Funde und Befunde aus den Nekropolen der TiszapolgárKultur. Als Ergebnisse dieser Untersuchungen setzten wir mit Recht voraus, dass die späte oder späteste bekannte lengyelzeitliche Belegung des Gräberfeldes von Alsónyék in die Zeit der frühen Belegung der Tiszapolgár-Gräberfelder datiert werden dürfte. ${ }^{18}$

Die gegenwärtige Lage der chronologischen Untersuchungen der Lengyel-Kultur fasste zuletzt R. Gleser im interregionalen Vergleich zusammen. ${ }^{19}$

\subsection{Forschungsmethoden der vorliegenden Arbeit}

Die erneute Analyse der Grabkeramik der Lengyel-Kultur machte die ungewönlich große Zahl der im Gräberfeld von Alsónyék-Bátaszék geborgenen Befunde und Funde notwendig. Unsere erste Analyse der lengyelzeitlichen Grabkeramik stammt aus 2007, als wir versuchten, die aus den Nekropolen, Gräbergruppen und -gruppierungen der „Altgrabungen“20 vorliegenden spätneolithisch-frühkupferzeitliche Fundmaterialien typologisch und relativchronologisch zu gliedern. Als Materialbasis dienten dazu 658 Bestattungen aus 628 Gräbern von zwölf südtransdanubischen Fundorten. ${ }^{21}$ Wir suchten auch nach den chronologischen Zusammenhängen der südöstlichen Lengyel-Gruppe mit den nördlich und westlich benachbarten Lengyel-Gruppen. Tatsache ist es auch heute noch, „,dass wir in Südtransdanubien nur über belegungsstratigraphischen Daten aus Nekropolen verfügen, während aus dem westlichen Verbreitungsgebiet der Lengyel-Kultur in erster Linie Siedlungsfunde zur Verfügung stehen. In diesen Fällen ist meist kaum zu entscheiden, ob es sich wirklich um geschlossene Befunde handelt." 22

Die Grundlage dieser Untersuchungen stellten die Merkmalanalyse und Seriation bzw. die archäologische vergleichende Methode dar. Mit Hilfe der metrischen Methode wurden die Merkmalgruppen definiert und mit diesen typologischen Gruppen eine belegungschronologische Abfolge der Bestattungen in den einzelnen Nekropolen sowie eine relativchronologische Parallelisierung der Gräberfelder versucht. Die kombinatoranalytischen Untersuchungen wurden in erster Linie anhand der Profilformen und Verzierungen der Grabkeramik vorgenommen. Das System der Profilformen umfasste zwei Klassen (Hochgefäße und Breitgefäße, sieben Gattungen: Fußgefäße, Becher, Näpfe, Schultergefäße, Butmir-Gefäße, Schüsseln und Schalen) sowie Serien, Formengruppen, Varianten und Untervarianten. Die Gefäßverzierungen wurden nach Motiven, Motivgruppen und Dispositionen der Verzierungen auf den Keramikgegenständen untersucht. ${ }^{23}$ Als Ergebnisse dieser älteren Forschungen stellten wir fünf Belegungsperioden (Lengyel 1a1, Lengyel 1a2, Lengyel 1b, Lengyel 2a1 und Lengyel 2a2) und drei Belegungshorizonte, frühe und späte Belegunshorizonte bzw. den Übergangshorizont zwischen ihnen dar. Die Ergebnisse unserer Analyse, auch im Verhältnis zu den steinernen Schaftlochaxttypen und den Kupferfunden in den Gräbern, bestätigten auch die Resultate der Seriation und der bisherigen 14C-Untersuchungen. ${ }^{24}$ Im Fall der vorliegenden Arbeit handelt es sich um einen Versuch, Synchronismen unter den verschiedenen Regionen der Lengyel-Kultur des 5. Jahrtausends auf Grund der Gräberfeldanalyse feststellen zu können. Ein solcher Versuch stammt jüngst von R. Gleser, als er versuchte, ,Synchronismen zwischen Süddeutschland und dem mittleren Donauraum mit Hilfe der bislang entworfenen Chronologieschemata und mit den Serien von AMS-Daten der Lengyel-Kultur“ zu bestimmen. Er behauptet, dass ,,im direkten Vergleich dieser Chronologiesysteme Unterschiede bei der absoluten Datierung bestimmter Stufen und Phasen von zum Teil mehreren Jahrhunderten festzustellen sind““. ${ }^{25}$ Diese Feststellung kann auch im Fall der verschiedenen Chronologiesysteme der Lengyel-Kultur in der Slowakei, Mähren, Österreich und Ungarn akzeptiert werden, ebenso wie „,rotz mehrerer, in Konkurrenz zueinander stehender Entwürfe, die zu unterschiedli-

${ }^{18}$ ZALAI-GAÁL 2014.

${ }^{19}$ GLESER 2012, 36: „Die Genese wird im sog. Vor-Lengyel-Horizont Westungarns und der Südwestslowakei vermutet. Darauf folgen die Stufe Prolengyel I (Bíňa-Bicske) und Protolengyel II (Lužianky-Sé), deren Funde in Niederösterreich in die Stufe Ia0 bezeichnet werden. Darauf folgen verschiedene Stufen und Phasen mit polychrom bemalter Keramik. Die Stufe mit weiß bemalter Keramik wird danach in zwei Phasen gegliedert (MOG IIa/MMK IIa/Lengyel II, Phase Pečeňady, Phase Moravany-Brodzany-Szob, Lengyel III/ MMK IIb). Darauf folgt ein Horizont mit unbemalter Keramik (Wolfsbach-Stufe/MOG IIb/MMK IIc/Lengyel IV oder Nitra-Stufe). Das so genannte Epi-Lengyel (Bisamberg-Oberpullendorf/klassisches Jordanow/klassisches Balaton-Lasinja I/Lengyel IV bzw. V) oder klassische Ludanice-Stufe schließt die Sequenz ab.“

${ }^{20}$ Schanzwerk von Lengyel, Szekszárd-Ágostonpuszta, Zomba-Paradicsompuszta, Pári-Altacker, Mórágy-Tűzkődomb, Györe, Zengővárkony 1-14b, Pécsvárad, Villánykövesd.

${ }^{21}$ ZALAI-GAÁL 2007a, 9, Tab. 9; ZALAI-GAÁL 2008b.

22 ZALAI-GAÁL 2007a, 9.

${ }^{23}$ ZALAI-GAÁL 2007a.

${ }^{24}$ ZALAi-GAÁl 2007a, Tab. 9; ZALAI-GAÁL 2007b, Abb. 4.

${ }^{25}$ GLESER 2012, 35 
chen und wiederholt abgeänderten Terminologien führten, die relative Chronologie der Lengyel-Kultur überregional als abgesichert gelten kann“. ${ }^{26}$

Auf Grund der restaurierten, gezeichneten und fotografierten Grabkeramiken von Alsónyék nahmen wir im Jahre 2011 eine erneute Analyse der südtransdanubischen Grabkeramik vor, um die relativchronologische Stellung dieses lengyelzeitlichen Gräberfeldes besser erkennen zu können. Dieses neue System umfasste dann 647 Keramiken aus 279 Bestattungen aus den Altgrabungen, während die Zahl der Grabgefäße von Alsónyék-Bátaszék nicht mehr als 215 aus 69 Bestattungen betrug. Diese neue Klassifizierung wurde anhand von neun Gattungen, 23 Serien, 59 Formengruppen und 126 Varianten durchgeführt. In diesem Fall handelt es sich im Wesentlichen um eine Integrierung von neuen Daten in das frühere analytische System. Es ist deswegen nicht klar, ob die Ergebnisse als relevant angesehen werden können. Somit konnten wir dagegen mit Recht voraussetzen, dass die untersuchten Grabungsflächen (10B und 5603) des Gräberfeldes von Alsónyék überwiegend während des späten Belegungshorizontes der südtransdanubischen Lengyel-Kultur benutzt wurden. Die Sequenz der seriierten südtransdanubischen Lengyel-Bestattungen, einschließlich jene von Alsónyék-Bátaszék, zeigt die Seriationstabelle 1 (die älteren Befunde unten). Auf der rechten Seite der Tabelle sind die Gräber von Alsónyék-Bátaszék rot (Fundstelle 10B) bzw. blau (Fundstelle 5603) markiert. ${ }^{27}$

Als ein Ergebnis dieser früheren Untersuchungen konnten wir die Verteilung der Gräber nach Belegungshorizonten in den größten Lengyel-Nekropolen wie folgt skizzieren (Diagramm 1).

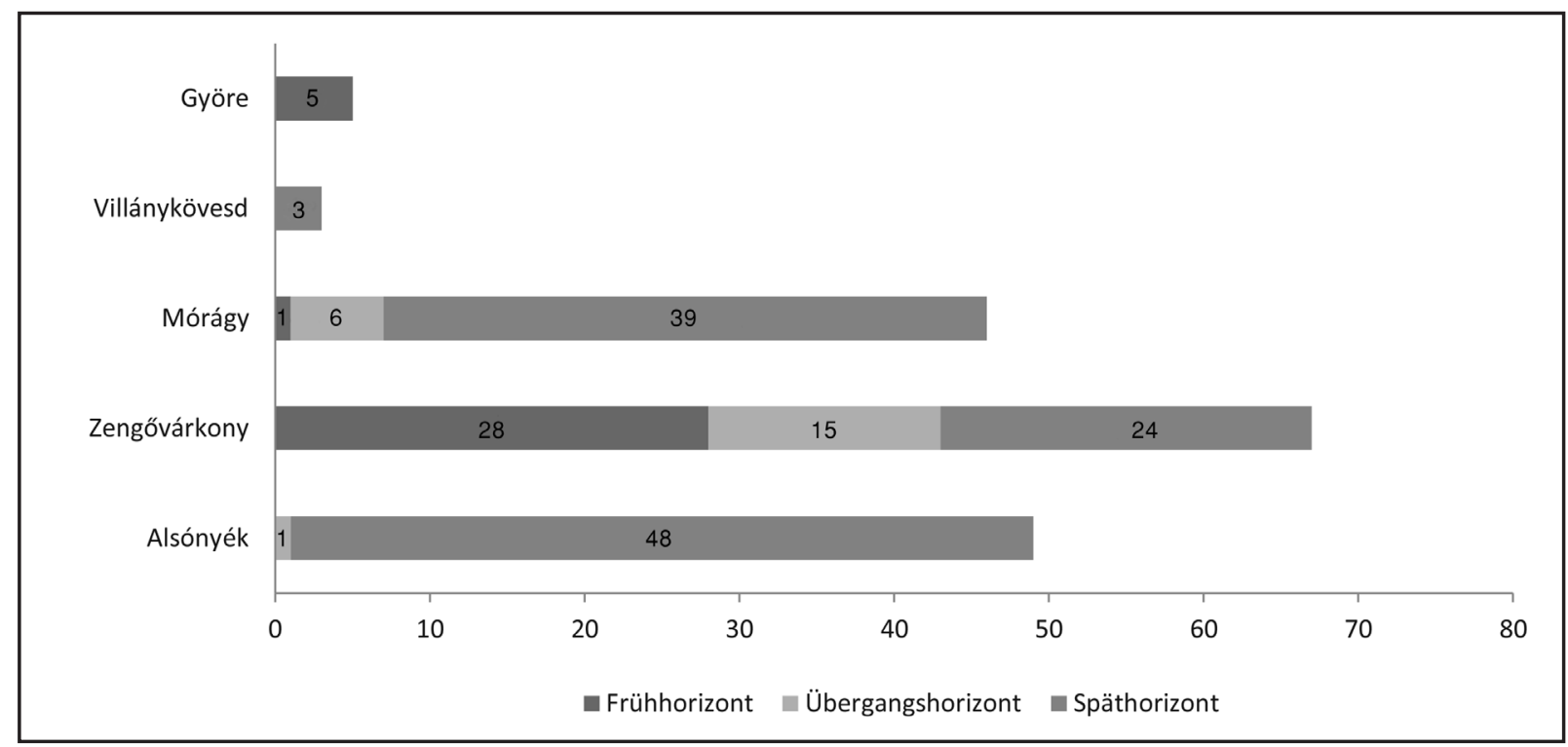

Diagramm 1. Verteilung der Gräber nach Belegungshorizonten in den größten Nekropolen der Lengyel-Kultur anhand der früheren Analysen

\subsection{Quellenbasis und Quellenlage der vorliegenden Arbeit}

Die in der vorliegenden Arbeit untersuchten Gräberfelder und diskutierten Fundorte der Lengyel-Kultur stellt die Verbreitungskarte 1 dar.

Verteilung und Vorkommen der jetzt untersuchten Befunde (Gräber) und Funde (Grabgefäße) nach Fundorten aus dem westlichen Karpatenbecken zeigt Diagramm 2.

Die Verteilung der analysierten Befunde nach den einzelnen Regionen der Lengyel-Kultur zeigt gleichzeitig ein uneinheitliches Bild. Die überwiegende Merhheit, 73,58\% $(n=323)$ der untersuchten Gräber bzw. 67,74 \% $(\mathrm{n}=716)$ der Keramikgegenstände, stammt aus Südtransdanubien (Altgrabungen und Alsónyék) und der Anteil der

\footnotetext{
${ }^{26}$ GLESER 2012, 36
}

\footnotetext{
${ }^{27}$ Manuskript, Sitzung des ERC-Teams im Mai 2013 in Budapest.
} 


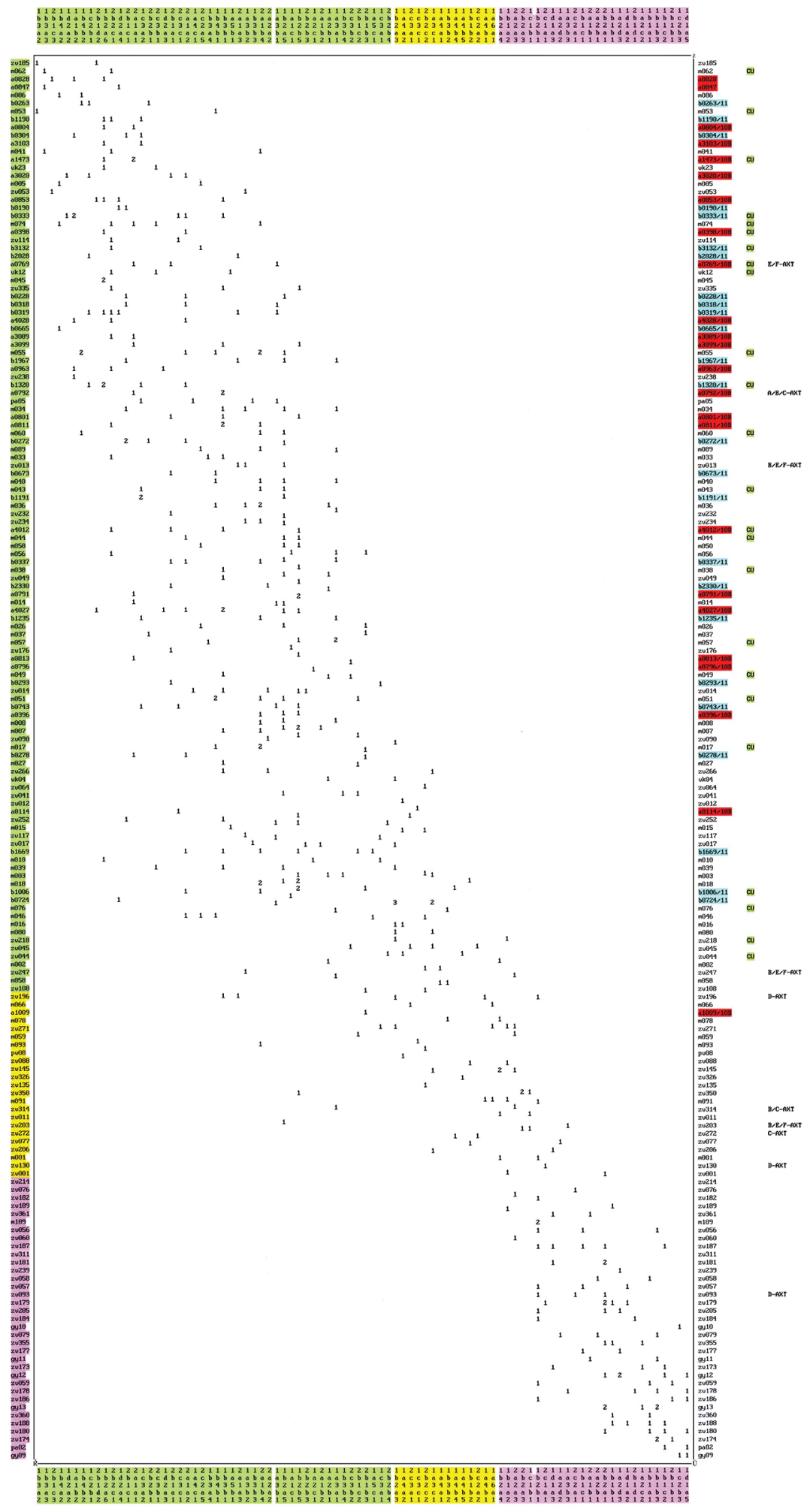

Seriationstabelle 1. Verteilung der südtransdanubischen Gräber der Lengyel-Kultur aus den Altgrabungen und von Alsónyék-Bátaszék nach Belegungshorizont (rot - Gräber der Fundstelle 10B, blau - Gräber der Fundstelle 5603 von Alsónyék-Bátaszék) 


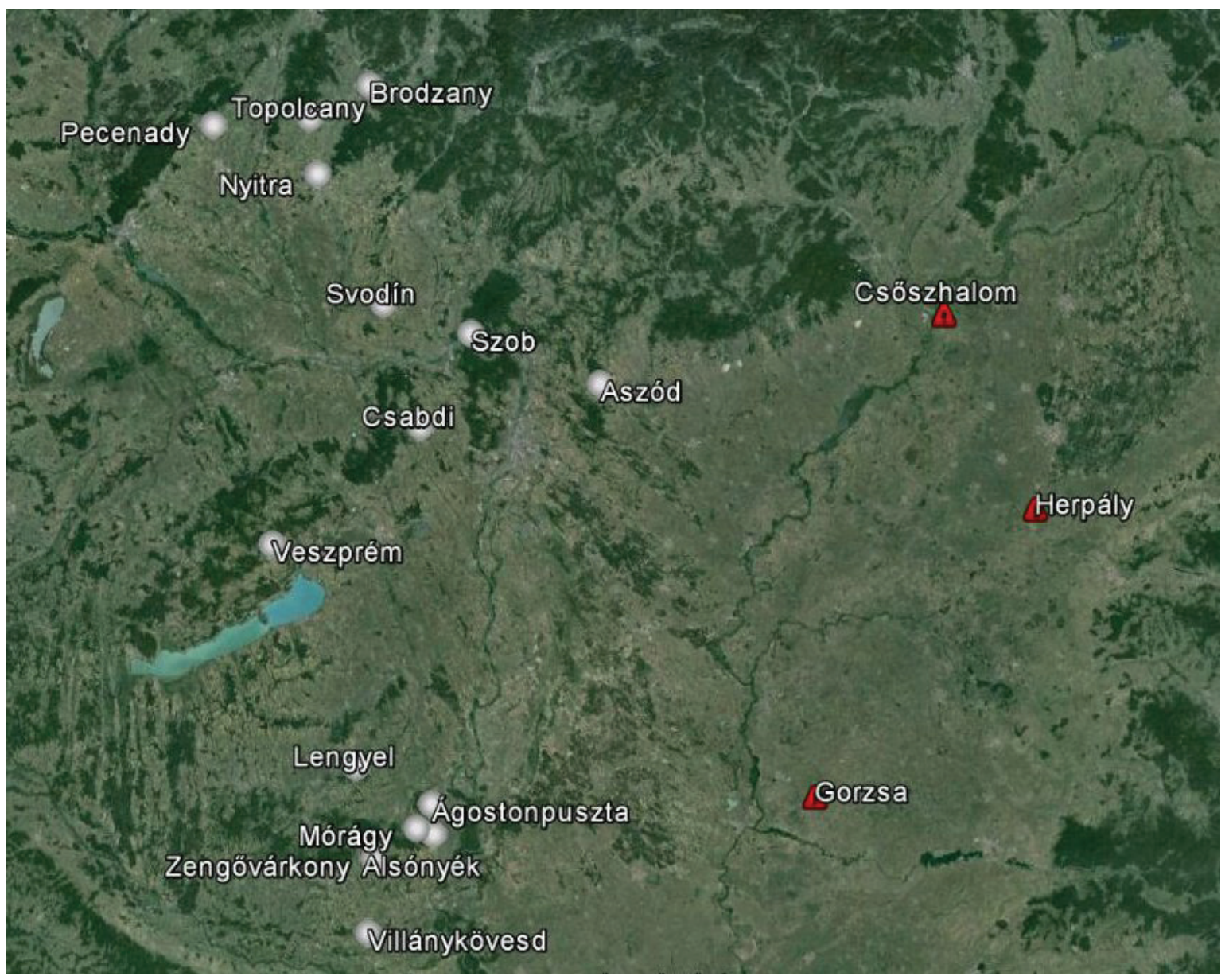

Verbreitungskarte 1. Untersuchte Gräberfelder und die diskutierten Fundorte der Lengyel-Kultur

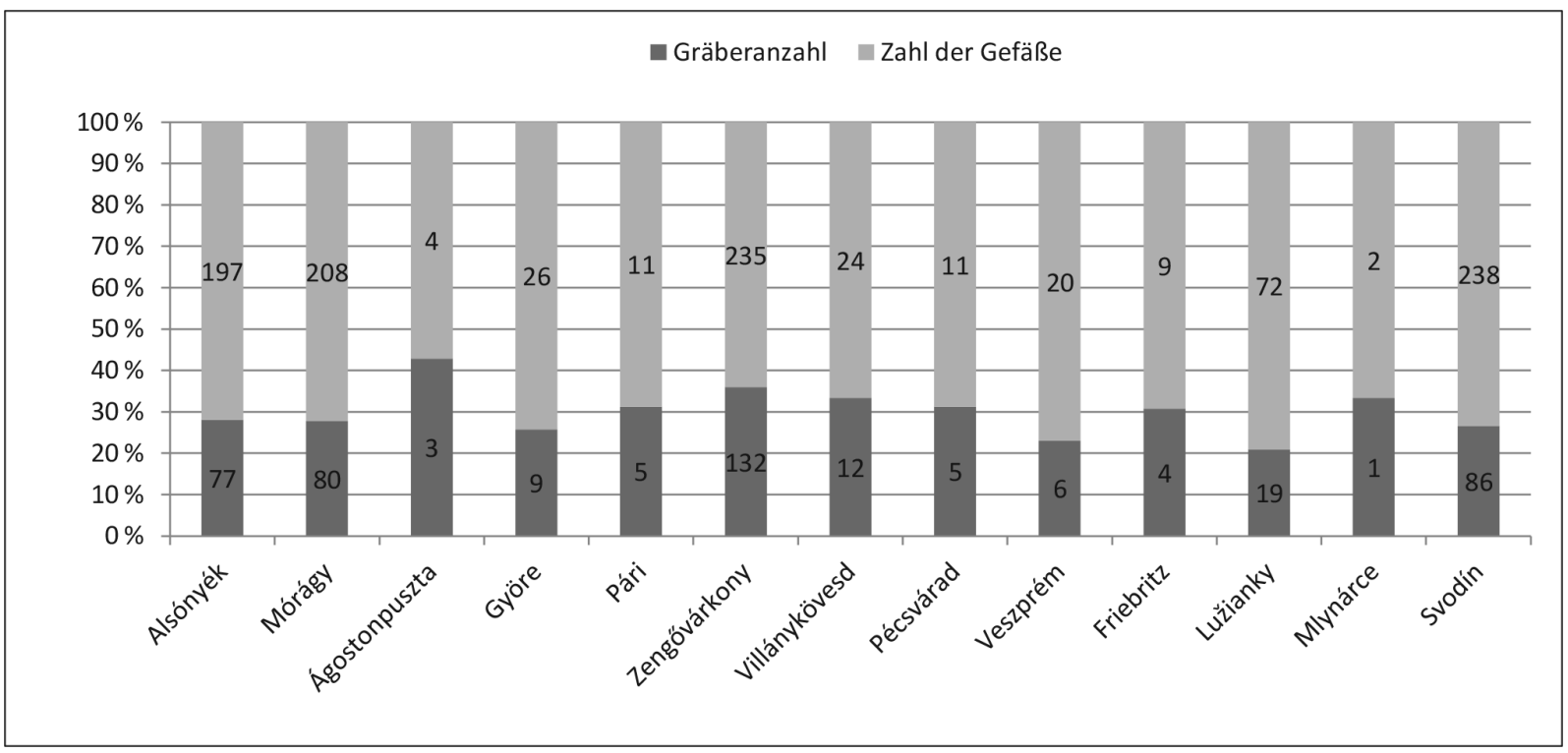

Diagramm 2. Verteilung der untersuchten Befunde und Funde in den Nekropolen der Lengyel-Kultur 


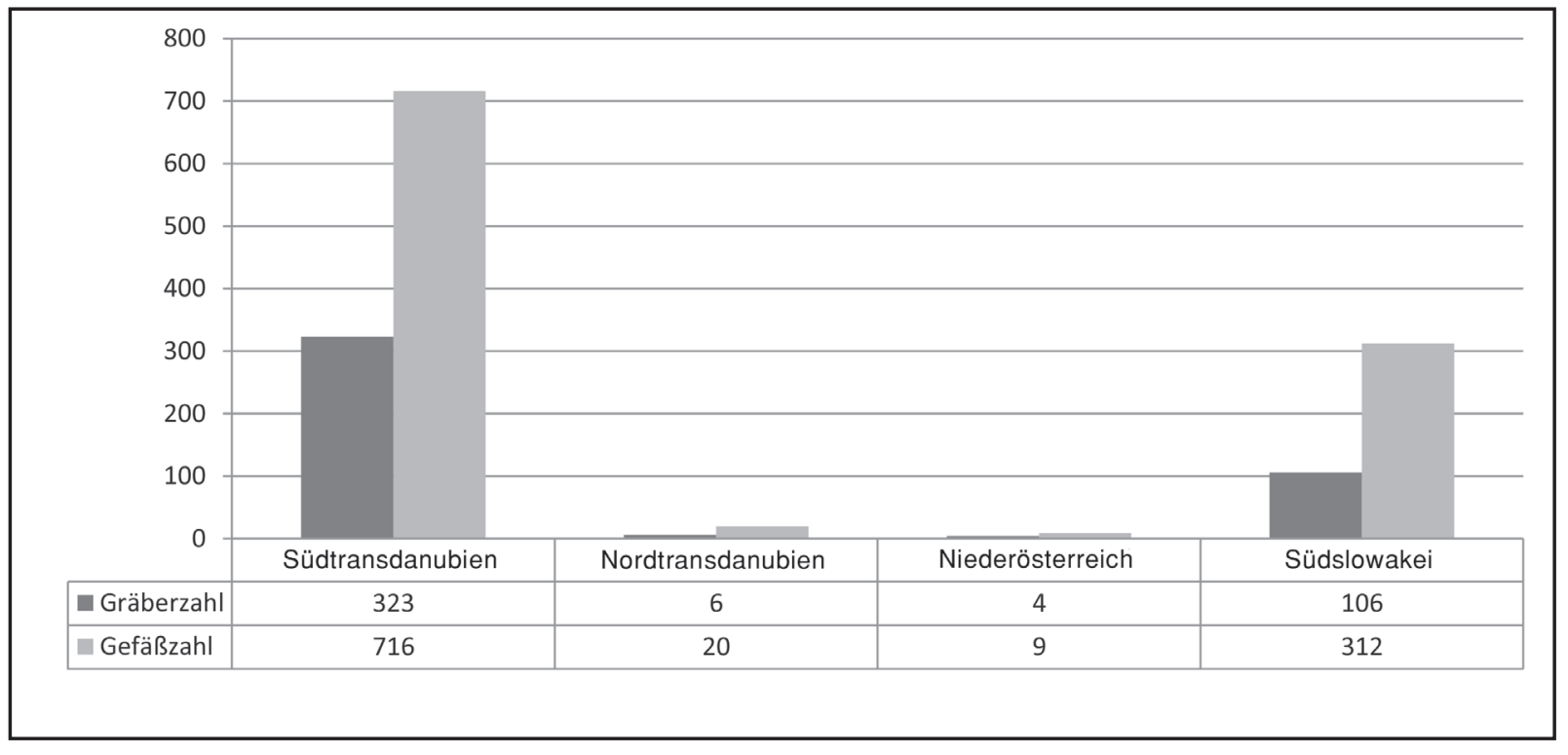

Diagramm 3. Vorkommen und Verteilung der untersuchten Befunde und Funde nach Verbreitungsregionen der Lengyel-Kultur

aus der Südwestslowakei (Lužianky-Gruppe und Svodín/Szőgyén) stammenden und studierten Befunde und Funde sind durch einen Prozentsatz von 24,15 \% $(n=106)$ bzw. 29,52 \% $(n=312)$ vertreten. Die Gräbergruppe vom nordtransdanubischen Fundort Veszprém-Jutasi út ergab sechs Bestattungen $(1,37 \%)$ mit 20 Keramikgefäßen $(1,89 \%)$ für unsere Analyse und aus der niederösterreichischen Gräbergruppe von Friebritz konnten wir nicht mehr als vier Bestattungen $(0,91 \%)$ mit 9 Keramikgegenständen $(0,85 \%)$ in den Katalog aufnehmen. Aus dem südslowakischen Lužianky (Sarlóskajsza) wurden Funde außer Menschengräbern auch aus Kultgruben und symbolischen Bestattungen in die Datenbasis aufgenommen, da es sich auch in diesen Fällen um geschlossene Befunde handelt. Die Verteilung der untersuchten Befunde und Funde nach Regionen der Lengyel-Kultur stellt Diagramm 3 dar.

\section{KLASSIFIKATION DER LENGYELZEITLICHEN GRABGEFÄSSE VON ALSÓNYÉK-BÁTASZÉK}

Im Fall der erneuten Merkmalanalyse der Grabkeramik der Lengyel-Kultur handelt es sich schon nicht um eine Ergänzung eines älteren typologischen Systems, sondern um eine neue Systemstruktur. Es war also absolut notwendig, die Maßbezeichnungen aller untersuchten Keramiken neu festzustellen, um sie in einem einheitlichen kombinatoranalytischen System klassifizieren zu können. Die Profilzeichnungen und die Beschreibungen der Exemplare aus den Altgrabungen standen dazu schon zur Verfügung. Nur ein Teil der Gefäße von Alsónyék-Bátaszék wurde gezeichnet und die überwiegende Mehrheit von ihnen fotografiert. Die Maßbezeichnungen wurden in diesen Fällen direkt an den Gefäßen bestimmt. Im Fall der Funde anderer Nekropolen der Lengyel-Kultur wurden die vergrößerten veröffentlichten Profilzeichnungen zur Bestimmung der Maßbezeichnungen verwendet. ${ }^{28}$ Die zweite Gruppe der Basis zur Klassifizierung der behandelten Gefäße stellen die Verzierungen dar.

Die Untersuchung der technologischen Eigenschaften der Scherben von Alsónyék-Bátaszék wird ein Gegenstand der kompletten Bearbeitung des ausgegrabenen Fundmaterials sein. Die technologischen Merkmale sind in relativchronologischer Hinsicht oft nicht immer kulturspezifisch, sie sind eher Folgeprodukt der in der Umgebung

${ }^{28}$ Györe: ZALAI-GAÁL-ÓDOR 2008; Veszprém-Jutasi út: REgenYe 2006; Friebritz-Süd: NeUgEBAUER-MARESCH et al. 2002; Svodín: DemJÁn 2010; Lužianky und Mlynárce: NovotnÝ 1962. 
der Fundstelle vorhandenen Rohmaterialplatte. Struktur und Farbe der Gefäße von Alsónyék-Bátaszék unterscheiden sich im Wesentlichen nicht von denen der aus den Altgrabungen Südtransdanubiens stammenden Gegenstände. ${ }^{29}$ Die Fußgefäße, dreigliedrigen Schultergefäße, Näpfe, Butmir-Gefäße und Schüssel sind gewöhnlich grobkörnig und dickwandig, während die drei- und zweigliedrigen bzw. kugeligen Becher und Schalen dünnwandig, mittelmäßig körnig, feinkörnig oder fein geschlämmt sind.

Die meisten Archäologen führten die typochronologischen Analysen auf Grund der Gefäßformen durch. Sie können nach vielen Gesichtspunkten klassifiziert werden. „Man rückt nur die Merkmale ins Blickfeld, welche hinsichtlich der Fragestellung relevant sind und auf deren Grundlage die sog. künstlichen und natürlichen Klassen ausgearbeitet werden können.“30 „Im Fall der künstlichen Klassen werden die formtypologischen Merkmale der Keramik nach einheitlicher Wertordnung analysiert. Die natürlichen Klassen stellen nach M. Dohrn-Ihmig solche Gegenstandgruppen dar, deren Merkmale miteinander auf hohem Niveau korrelierbar sind. “31 Die prähistorische Typologie stützt sich also auf ausgewählte Merkmale, die es erlauben, die Funde voneinander zu unterscheiden. Übereinstimmungen und Ähnlichkeiten der Merkmale zeigen dabei typologisch zusammengehörige Funde auf. Die untersuchten Formen von Gebrauchsgegenständen waren dabei aber einer ständigen Veränderung unterworfen. Deshalb wird also eher ein komplexer dynamischer Prozess mit Hilfe der Typologie vereinfacht dargestellt.

Die untersuchten Keramiken der Lengyel-Kultur aus dem westlichen Karpatenbecken wurden in einem System gruppiert, das aus zwei Klassen (Klasse 1 - Hochgefäße, Klasse 2 - Breitgefäße), acht Gattungen (1a - Fußgefäße, 1b - dreigliedrige Becher und Schultergefäße, 1c - doppelkonische Becher, 1d - kugelige Becher, 1e Näpfe, 2a - Butmir-Gefäße, 2b - Schüsseln, 2c - Schalen), 29 Serien, 146 Typengruppen und 384 Typen besteht. Vorkommen und Verteilung der keramischen Formen in Alsónyék und aus dem übrigen Verbreitungsgebiet der Lengyel-Kultur zeigt Diagramm 4. Diese Daten werden sich nach der Bearbeitung des gesamten Fundmaterials von Alsónyék-Bátaszék selbstverständlich verändern.

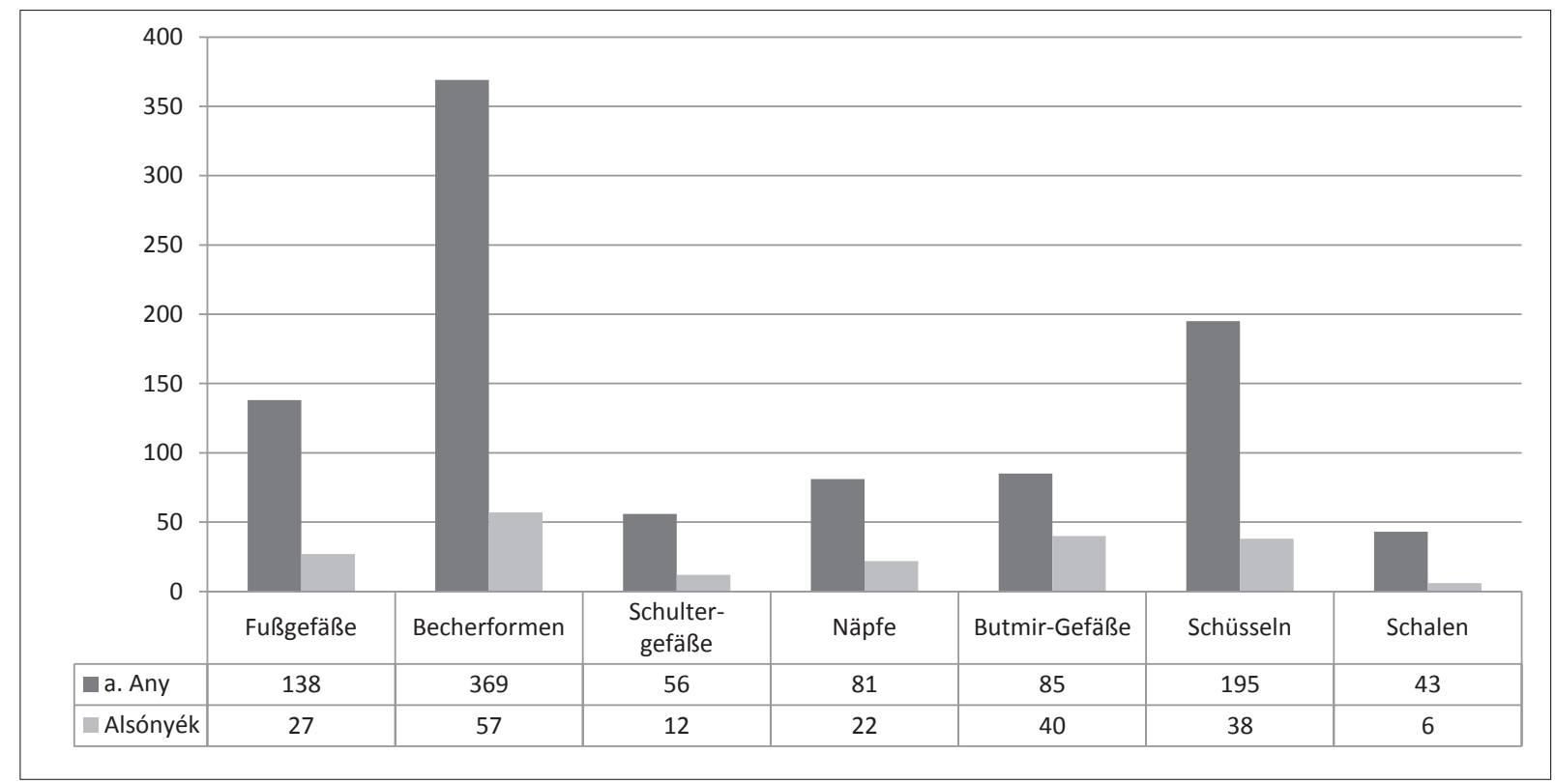

Diagramm 4. Verteilung der untersuchten keramischen Formen in Alsónyék und aus dem weiten Verbreitungsgebiet der Lengyel-Kultur

${ }^{29}$ ZALAI-GAÁL 2007a, 33-34.

${ }^{30}$ Stehli-Zimmermann 1980, 152; Zalai-GaÁL 2007a, 35.
${ }^{31}$ Dohrn-Ihmig 1974; StehLi-Zimmermann 1980, 151. 


\subsection{Klasse 1 - Hochgefäße}

\subsubsection{Gattung la-Fußgefäße}

Die Gefäße dieser Gattung können in zwei formale Teile gegliedert werden: eine Schüssel oder Schale bzw. ein Hohlfuß. Es wurden insgesamt 165 ganze oder meßbare Exemplare von Fußgefäßen in die Analyse aufgenommen. In 49 Fällen sind nur die Hohlfüße oder die Schüsselteile der Fußgefäße erhalten (Diagramm 4).

Die typologischen Einheiten der Fußgefäße haben wir mit Hilfe der Kombinationen verschiedener Varianten der Schüsselformen und Hohlfüße und der Untersuchung der Profilformen bestimmt. Die Klassifizierung wurde anhand folgender Indexwerte durchgeführt: Die Serien von Fußgefäßen stellten wir mit Hilfe des Indexwertes A1 (Höhe des Hohlfußes/Höhe der Schüssel) fest. Zur Bestimmung der Typengruppen verwendeten wir folgende Indexwerte: Die Hohlfüße können grundlegend durch Vergleich der Indexwerte fa (Hohlfußhöhe/größte Breite des Fußes) und fb (Halsbreite/größte Breite des Fußes) gruppiert werden, während die Schüsselteile durch den Vergleich der Indexwerte Sa (Schüsselhöhe/größte Schüsselbreite) und Sb (Mündungsbreite/Halsbreite). Zur Bestimmung der Typen (Varianten) innerhalb der einzelnen Typengruppen berücksichtigten wir in erster Linie Index Sc (Höhe des Unterteils der Schüssel/Höhe des Oberteils der Schüssel) und die Profilform. Die Maßbezeichnungen der Fußgefäße zeigt Abb. 2.

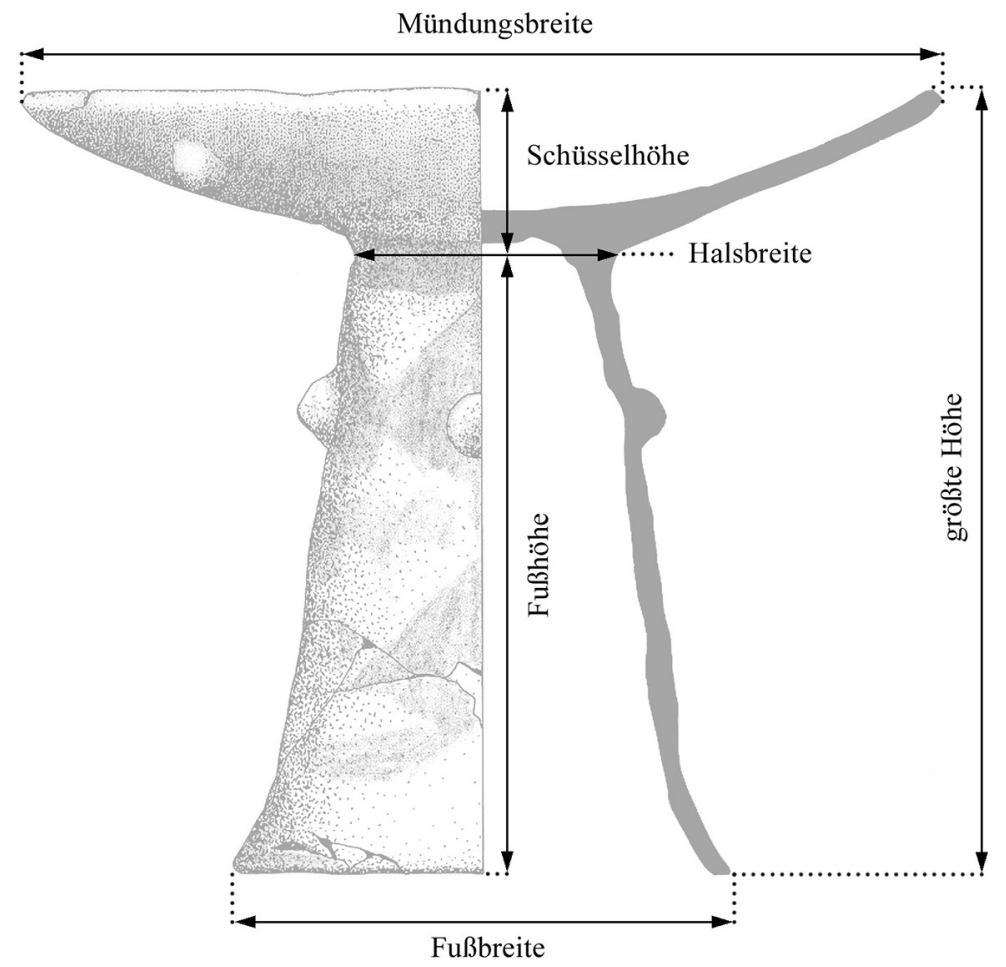

Abb. 2. Maßbezeichnungen eines Fußgefäßes auf den verschiedenen Ebenen

Auf diese Weise konnten wir die untersuchten lengyelzeitlichen Fußgefäße in vier Serien, 29 Typengruppen und 67 Varianten (Typen) trennen. In Alsónyék sind 10 durch diese Typengruppen und 16 durch die Typen vertreten. In der vorliegenden Arbeit zeigen wir nur die charakteristischen Beispiele einzelner Typengruppen aus Alsónyék auf Abbildungen vor. Die Funde der Fundstelle Alsónyék 10B werden auf den Abbildungen mit an-, jene der Fundstelle Alsónyék 5603 mit az- bezeichnet.

Die Exemplare der Typengruppe 1a1a von Fußgefäßen können durch extrem niedrigen, konischen Hohlfuß und breitkonische, niedrige Schüsselpartie gekennzeichnet werden $(A b b .3 a-c)$. Sie bilden vier Varianten unterein- 

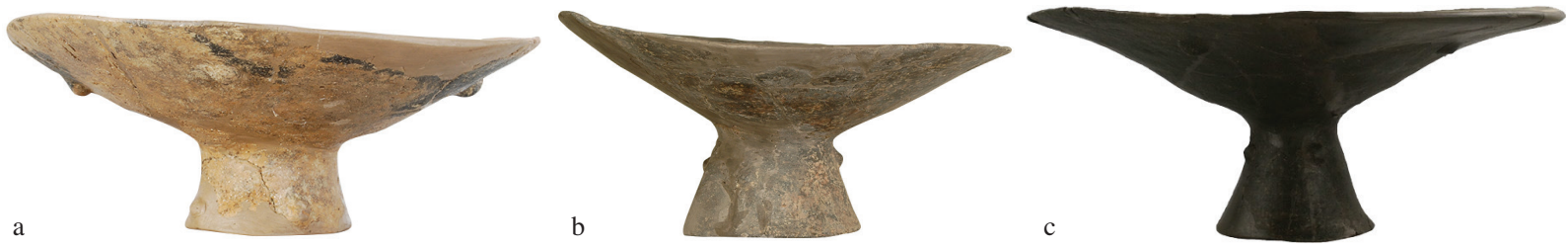

Abb. 3a-c. Alsónyék-Bátaszék. Fußgefäße der Typengruppe 1a1a.

a-b: Variante 1a1a3 aus den Gräbern an828 und an963; c: Variante 1a1a4, Grab az304

ander und treten außerhalb des Gräberfeldes von Alsónyék noch in Zengővárkony (Gräbergruppen 6c, 6d, 8b, 9, 10) und Mórágy auf.

Für die Typengruppe 1a2e (mit einer Variante) sind höherer und konischer Hohlfuß und weit ausladende, höhere Schüsselpartie typisch (Abb. $4 a$ ). Dieser Gefäßtyp ist nur noch aus Zengővárkony (Gräbergruppe 6b) belegt.

Die Keramiken der Typengruppe 1a2j (mit vier Varianten) besitzen höheren, sich nach unten stark ausweitenden Hohlfuß und höhere, konische Schüssel $(A b b .4 b)$. Weitere Exemplare sind noch in Zengővárkony (Gräbergruppen 2b, 6c und 9), Pécsvárad, Mórágy und Veszprém-Jutasi út (im Weiteren: Veszprém) nachgewiesen.

Die Typengruppe 1a2k (vier Varianten) ist auch in Zengővárkony (Gräbergruppe 6c), Villánykövesd und Mórágy vertreten. Typisch sind für diese Gefäße der lange, sich unten ausbreitende Hohlfuß und die konische, breite Schüssel mit ausladendem Hals (Abb. 4c-f).
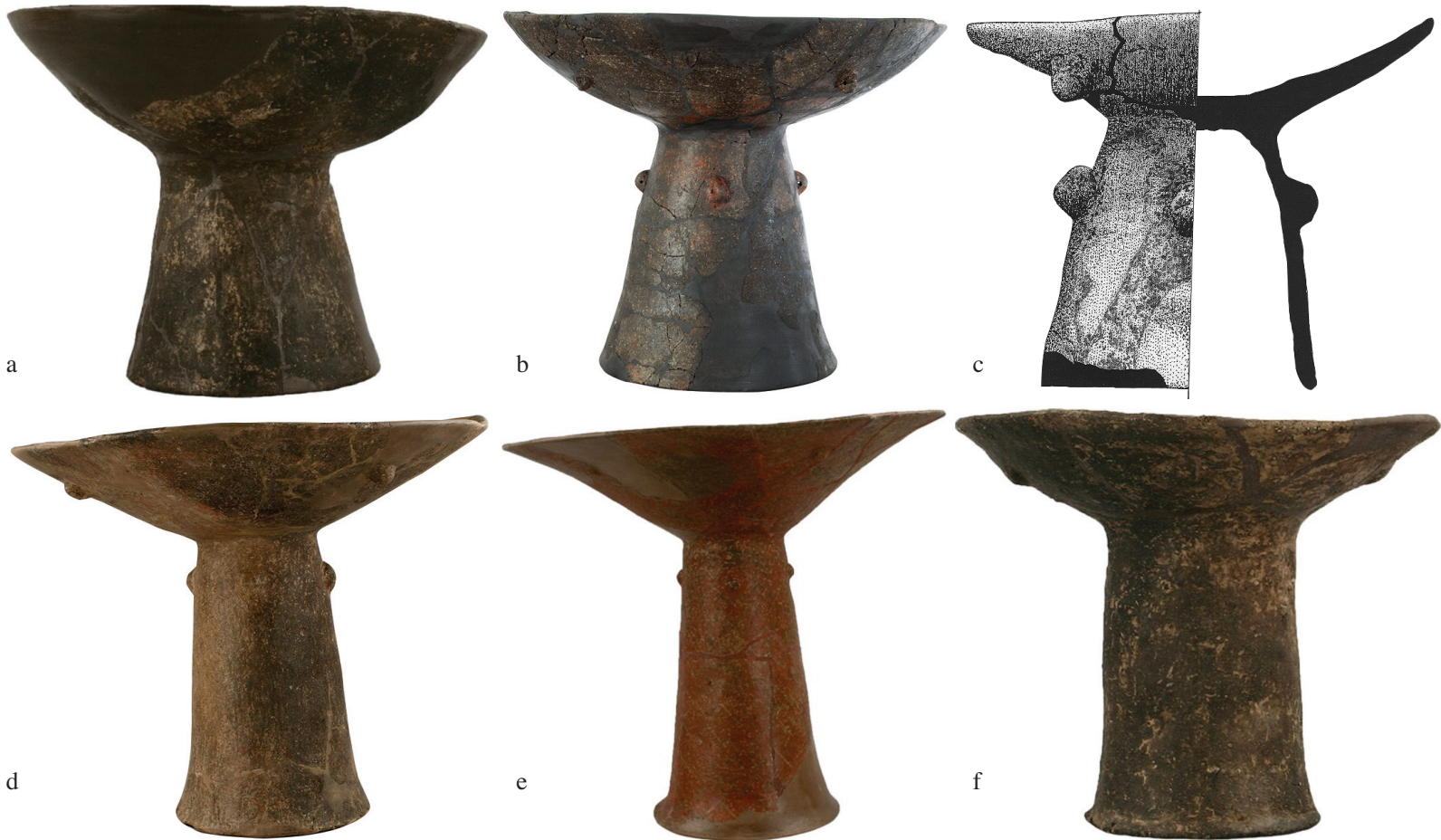

Abb. 4a-f. Alsónyék-Bátaszék. Fußgefäße der Typengruppen 1a2e, 1a2j und 1a2k.

a: Variante 1a2e1, Grab az337; b: Variante 1a2j2, Grab an3089; c: Variante 1a2k1, Grab an396; d: Variante 1a2k3, Grab az699; e: Variante 1a2k4, Grab az1191; f: Variante 1a2k5, Grab az1937

Auch die Typengruppe $1 a 2 l$ (mit drei Varianten) ist außer Alsónyék nur noch in Zengővárkony (Gräbergruppen $6 c, 9,13$ ) und Villánykövesd belegt. Die Hohlfüße dieser Gefäße weisen leicht profilierte oder ein wenig glockenförmig modellierte Gestalt auf, während die Schüsselpartie flach und breit ist (Abb. 5a-b). 


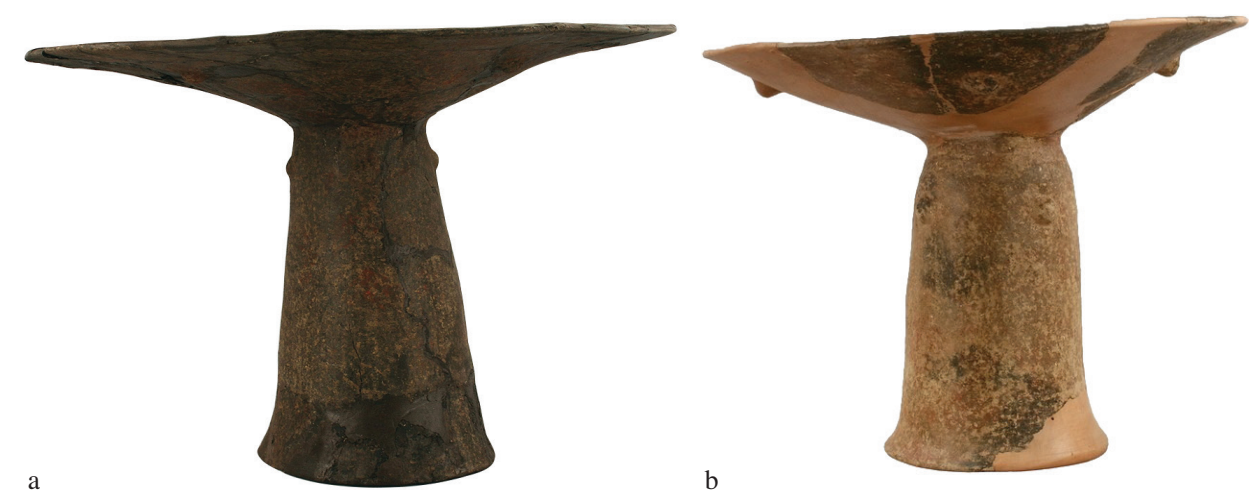

Abb. 5. Alsónyék-Bátaszék. Fußgefäße der Typengruppe 1a21. a: Variante 1a212, Grab az1320; b: Variante 1a214, az272

Die Fußgefäße der Typengruppe 1a3e (mit vier Varianten) mit breiterem, kürzerem und sich leicht profiliert ausladendem Hohlfuß und mit höherer, konischer Schüssel erscheinen auch in Zengővárkony (Gräbergruppen 9 bzw. 10) und Mórágy ( $A b b .6 e)$, während die Typengruppe 1a3i allein in Alsónyék auftritt $(A b b .6 b)$. Die mit noch breiterem und leicht profiliertem Hohlfuß und flacher, konischer Schüssel modellierten Exemplare der Typengruppe 1a3k sind auch in Lengyel, Zengővárkony (Gräbergruppen $6 \mathrm{a}, 6 \mathrm{c}, 8 \mathrm{~b}, 9)$ und Villánykövesd nachgewiesen $(A b b .6 c-d)$.

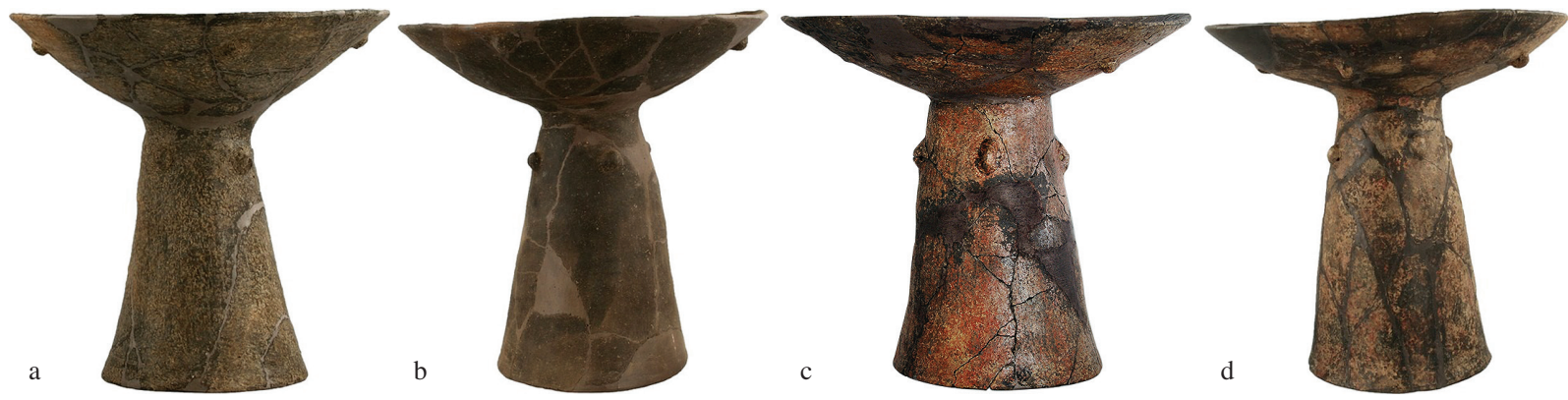

Abb. 6a-d. Alsónyék-Bátaszék. Fußgefäße der Typengruppen 1a3e, 1a3i und 1a3k.

a: Variante 1a3a2, Grab az1669; b: Variante 1a3i1, Grab az743; c: Variante 1a3k1, an4027; d: Variante 1a3k4, Grab az337

Das Artefakt aus dem Grab 5603/1669 von Alsónyék-Bátaszék stellt eine Sonderform (Typengruppe 1a4a) unter den Fußgefäßen dar (Abb. 7a). Die Typengruppe 1a4c, die auch in Zengővárkony (Gräbergruppen 2b, 6a, 6b, 9 bzw. 11) und Mórágy bekannt ist, wird in Alsónyék durch ein Exemplar mit langem und schlankem Hohlfuß bzw. mit hohem, konischem Schüsselteil vertreten $(A b b .7 b)$.

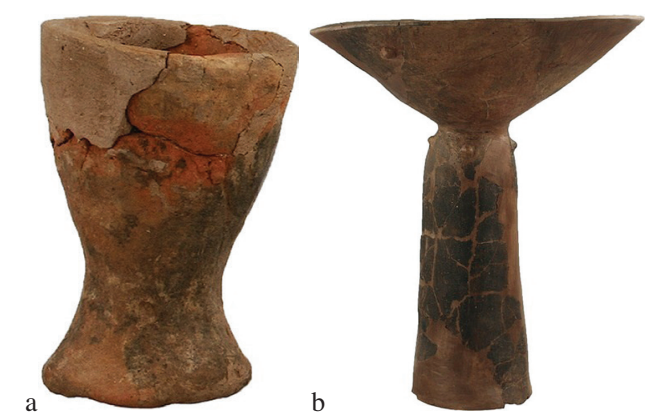

Abb. 7a-b. Alsónyék-Bátaszék. Fußgefäße der Typengruppen 1a4a und 1a4c. a: Variante 1a4a1, Grab az1669; b: Variante 1a4c5, Grab az665 
Die keramische Unterklasse von Bechern wird verschiedenartig definiert. R. Gleser rechnete zu dieser Gattung Keramiken, die einen Randdurchmesser mit einer Größe von 70 \% bis 130 \% der Höhe aufweisen. ${ }^{32}$ Ein weiteres Kriterium können auch die betonte Schulterpartie und ein Höhen-Breiten-Index von ca. $100 \%$ darstellen. ${ }^{33}$ Bei der erneuten Merkmalanalyse der lengyelzeitlichen Grabkeramik werden die drei-, zwei- und eingliedrigen Becher nach getrennten Gattungen untersucht.

\subsubsection{Gattung $1 b$-dreigliedrige Becher und Schultergefäße}

Auch die Exemplare unserer früheren Gattung 1d von Schultergefäßen oder Krügen ordneten wir wegen ihrer dreigliedrigen Formgebung zu dieser Gattung. Im untersuchten Gesamtmaterial befinden sich 381 dreigliedrige Gefäße. Von Alsónyék stammen davon 46 Exemplare: Es handelt sich dabei um 35 Becher und 11 Schultergefäße. Sie können nach den Längen-Breiten-Indexwerten (Index A1) in fünf Serien, nach den Bauchbreiten-Bodenbreiten-Indexwerten (Index D1) in 39 Typengruppen und nach den Indexwerten E1 (Höhe des Unterteils/Höhe des Oberteils) bzw. der Profilform in 118 Varianten eingeordnet werden. Die Maßbezeichnungen der dreigliedrigen Becher zeigt $A b b .8$.

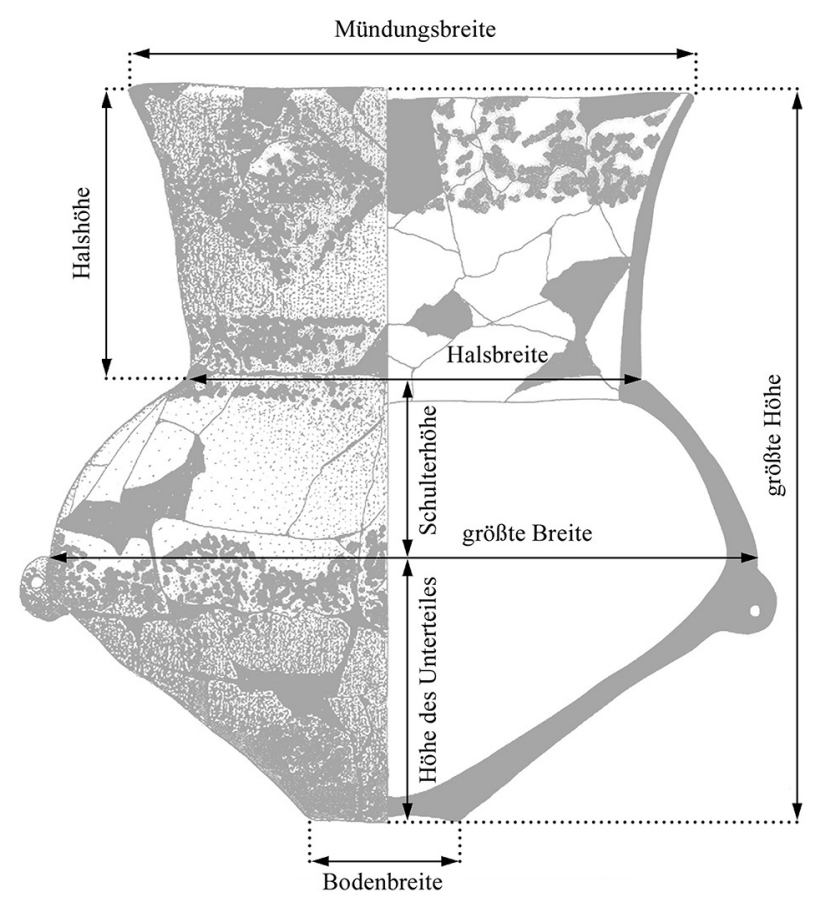

Abb. 8. Maßbezeichnungen eines dreigliedrigen Gefäßes auf den verschiedenen Ebenen

Es sind 13 Typengruppen dieser keramischen Gattung, die auch in Alsónyék vorhanden sind. Für die Becher der Typengruppe 1b1f (drei Varianten) sind niedriger Unterteil mit nach innen geschweifter Wandung, starker Bauchumbruch, gewölbte Schulterpartie und kurzer abgesetzter, ein wenig tichterförmig modellierter Hals typisch. Diese Typengruppe ist mehrheitlich in Mórágy vertreten (Abb. 9a). Auch die Typengruppe 1b2f (fünf Varianten) besitzt eine ähnliche Form, der Unterteil ist dagegen schlanker und die Schulter höher ausgestaltet. Solche Keramiken wurden auch in Mórágy und Zengővárkony (Gräbergruppen 2b, 5 und 6c) gefunden ( $A b b .9 b$ ). 

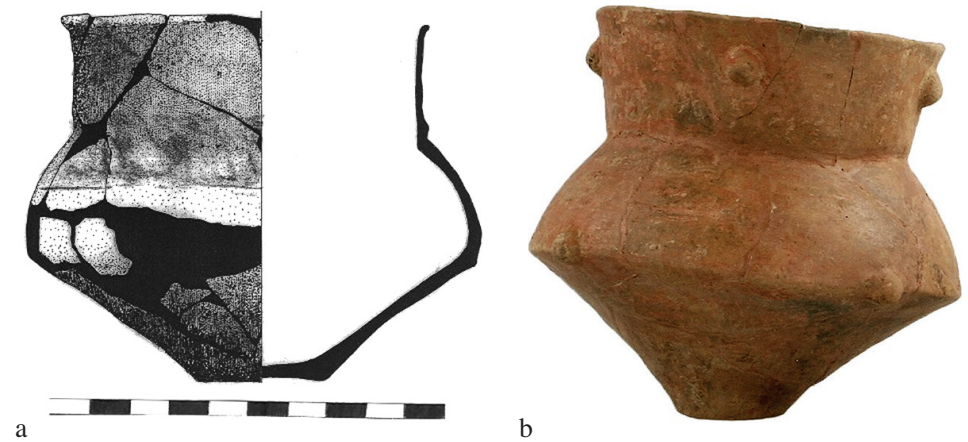

Abb. 9a-b. Alsónyék-Bátaszék. Dreigliedrige Gefäße (Becher) der Typengruppen 1b1f und 1b2f. a: Variante 1b1f1, Grab an114; b: Variante 1b2f4, Grab az673

Die Typengruppe 1b3c (fünf Varianten) kann mit breiterem Unterteil, mit niedrigerer Schulter und mit viel höherem Hals gekennzeichnet werden. Sie erscheint auch in Mórágy, Villánykövesd und Zengővárkony (Gräbergruppe 6d) (Abb. 10a-b).
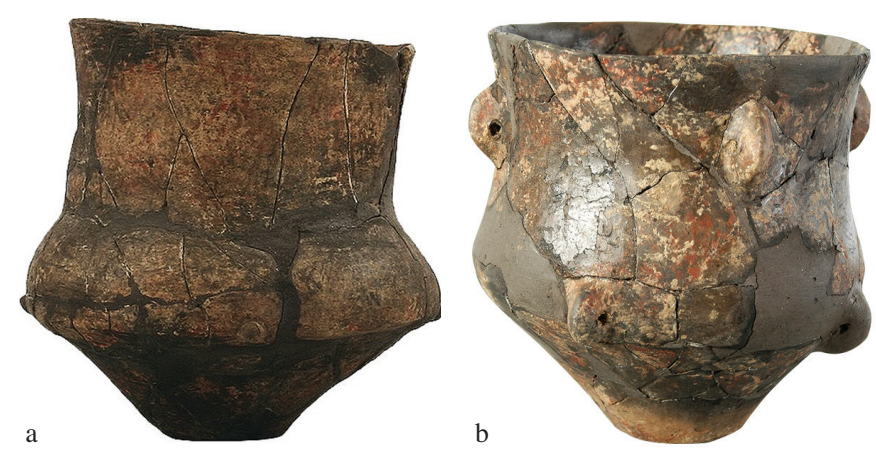

Abb. 10a-b. Alsónyék-Bátaszék. Dreigliedrige Gefäße (Becher) der Typengruppe 1b3c. a: Variante 1b3c2, Grab az272; b: Variante 1b3c3, Grab an799

Die Typengruppe 1b3e (fünf Varianten) weist einen höheren, leicht nach innen gezogen ausladenden Unterteil mit ähnlich hoher Schulterpartie auf und der Hals ist kürzer und trichterförmig. Die Exemplare der Typengruppe sind noch in Mórágy und Zengővárkony (Gräbergruppen 2b, 3b, 6b) vorhanden (Abb. 11a-c).

Schlankere und höhere Form bzw. niedrigere Schulter und höherer Hals sind für die Becher der Typengruppe 1b4f (mit drei Varianten) kennzeichnend. Auch diese typologische Einheit ist noch in Zengővárkony (Gräbergruppen 5, 6a, 6c, 6d, 9 bzw. 13) und Mórágy vertreten $(A b b .12 a-b)$. Die Becher der Typengruppe 1b4g (eine
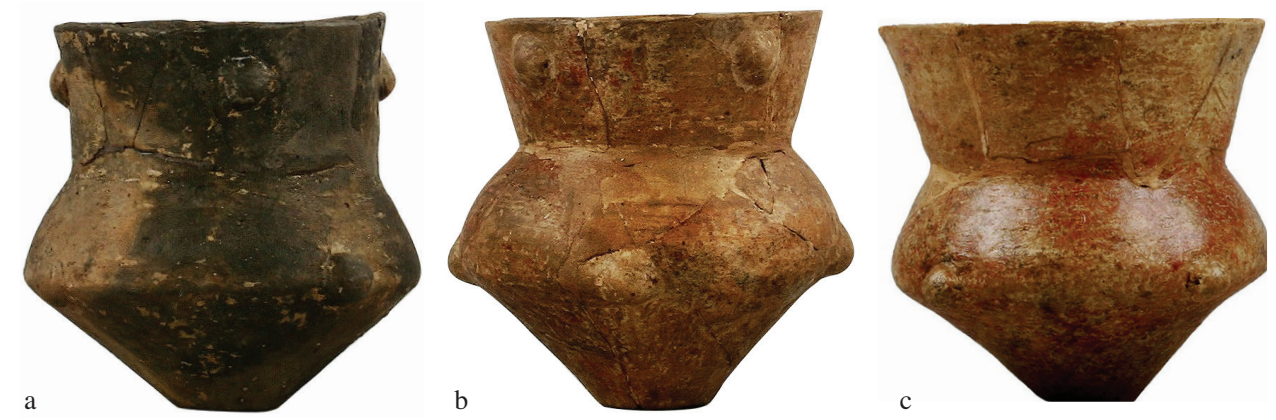

Abb. 11a-c. Alsónyék-Bátaszék. Dreigliedrige Gefäße (Becher) der Typengruppe 1b3e.

a: Variante 1b3e2, Grab az1190; b: Variante 1b3e5, Grab az1235 und Grab az2330 
Variante) besitzen dagegen einen niedrigeren und breiteren Unterteil und hohen, breiten, trichterförmigen Hals. Ein solches Exemplar befindet sich noch in Zengővárkony (Gräbergruppe 14a) (Abb. 12c). Die Gefäßpartie zeigt unterhalb des Halses eine doppelkonische Form der Typengruppe 1b4h (mit einer Variante) und der weniger abgesetzte Hals ist breit und leicht konisch ausgestaltet. Die Typengruppe erscheint auch in Mórágy (Abb. 12d).
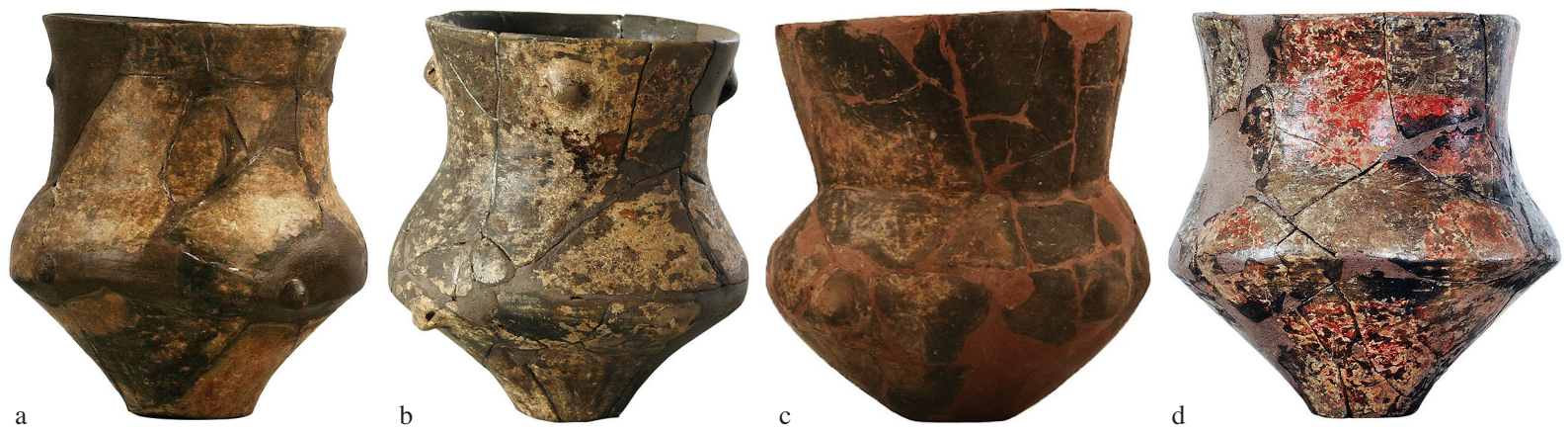

Abb. 12a-d. Alsónyék-Bátaszék. Dreigliedrige Gefäße (Becher) der Typengruppen 1b4f, 1b4g und 1b4h.

a: Variante 1b4f1, Grab az1320; b: Variante 1b4f2, Grab an828; c: Variante 1b4g1, Grab az264; d: Variante 1b4h1, Grab an4027

Für die fein ausgearbeiteten Exemplare der Typengruppe 1b4i (mit sechs Varianten) sind der schlanke, stark nach innen gezogener Unterteil, der starke Bauchumbruch, die gewölbte Schulter und der breitkonische lange Hals kennzeichnend. Diese keramische Form erscheint sowohl in Zengővárkony (Gräbergruppen 2b, 6a, 6c, 6d bzw. 11) und Mórágy als auch in Veszprém (Abb. 13a-b).
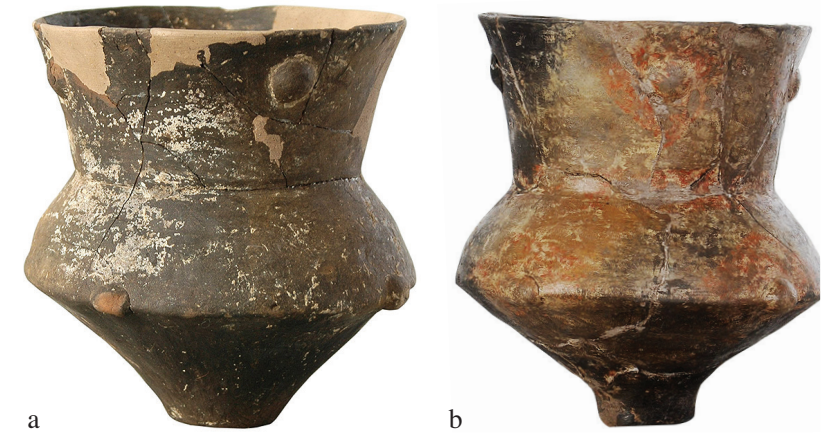

Abb. 13a-b. Alsónyék-Bátaszék. Dreigliedrige Gefäße (Becher) der Typengruppe 1b4i. a: Variante 1b4i2, Grab an813; b: Variante 1b4i5, Grab an796

Die Schultergefäße ähneln nach Formgebung den dreigliedrigen Bechern, sie besitzen dagegen eine viel größere Gestalt. Das Verhältnis der Höhe zur Breite ist wenigstens 1:3 und der Halsdurchmesser entspricht etwa der Hälfte des Bauchdurchmessers. ${ }^{34}$

Die Exemplare der Typengruppe 1b5i (mit zwei Varianten) haben einen hohen und konischen Unterteil, eine niedrige Schulter und einen mittelmäßig langen, leicht konischen Hals. Diese Gefäßform ist allein für das Gräberfeld von Alsónyék typisch (Abb. 14a-b).

Ebenso allein in Alsónyék kommt die Typengruppe 1b5j (mit zwei Varianten) vor. Es handelt sich um Keramiken, deren schlankerer Unterteil länger ist als die Schulterpartie, und der Hals wurde zylindrisch oder trichterförmig modelliert (Abb. $14 c-d)$.

Einen extrem niedrigen Unterteil, eine hohe und gewölbte Schulter und einen hohen, sich nach oben verengernden Hals mit ausladendem Rand besitzen die Exemplare der Typengruppe 1b5k (mit einer Variante) (Abb.

${ }^{34}$ STROBEL 1996, 22. 

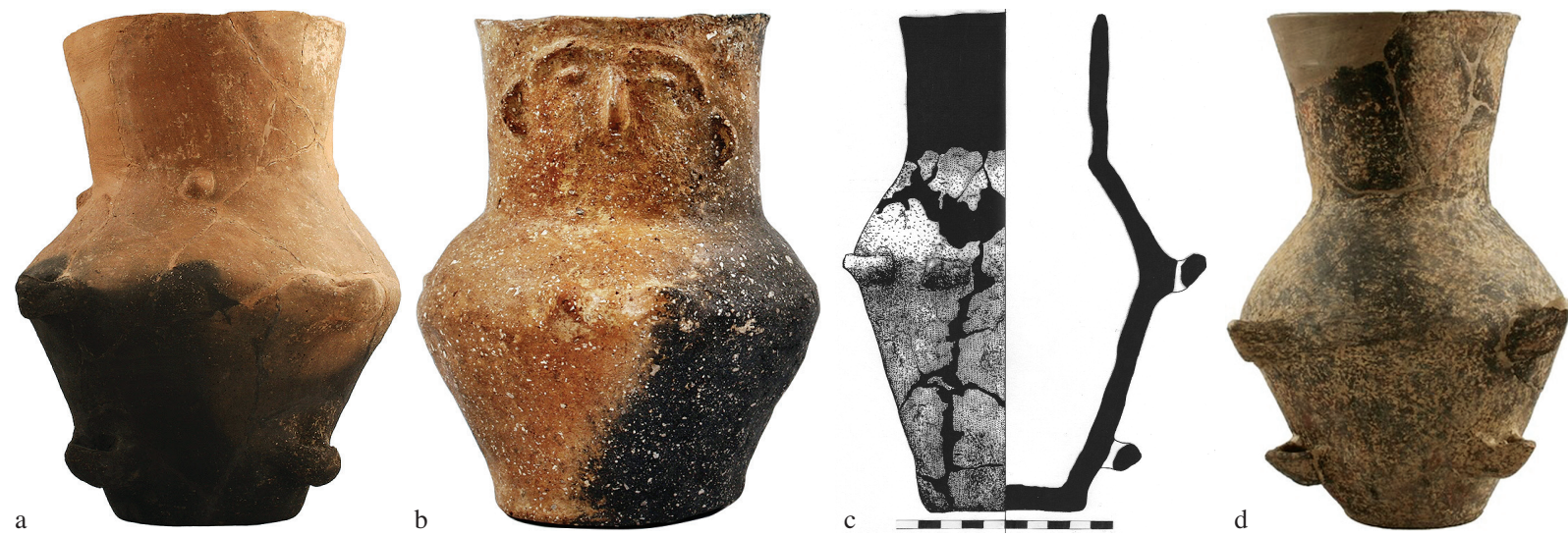

Abb. 14a-d. Alsónyék-Bátaszék. Dreigliedrige Gefäße (Schultergefäße) der Typengruppen 1b5i und 1b5j.

a: Variante 1b5i1, Grab az190; b: Variante 1b5i2, Grab az326; c: Variante 1b5j1, Grab an988; d: Variante 1b5j2, Grab az319
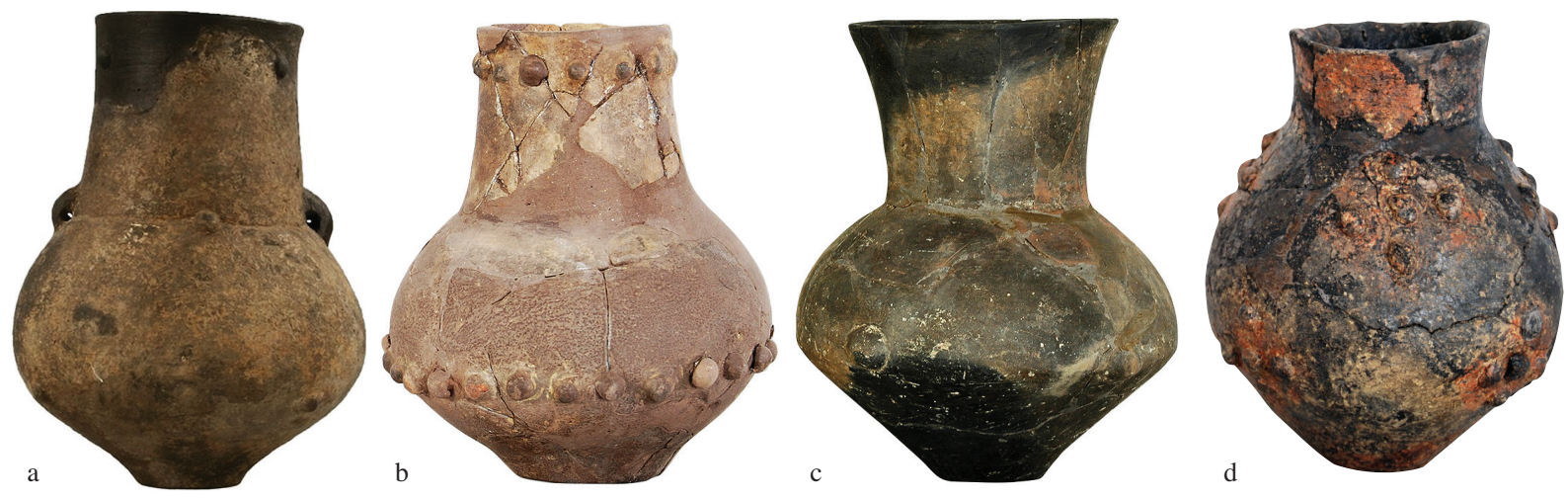

Abb. 15a-d. Alsónyék-Bátaszék. Dreigliedrige Gefäße (Schultergefäße) der Typengruppen 1b5k, $1 \mathrm{~b} 51$ und 1b5m. a-b: Variante 1b5k1, Grab az333 und Grab an3020; c: Variante 1b511, Grab an963; d: Variante 1b5m1, Grab an4011

$15 a-b)$. Auch das Schultergefäß aus dem Grab 352 der Gräbergruppe 13 von Zengővárkony vertritt diese Typengruppe.

Die Typengruppe 1b51 (mit zwei Varianten) kann mit gedrungenem Körper und hohem, trichterförmigem Hals charakterisiert werden. Der Bauchumbruch ist auch in diesem Fall kantig ausgebildet ( $A b b .15 c)$. Für die Typengruppe $1 \mathrm{~b} 5 \mathrm{~m}$ (mit zwei Varianten) ist dagegen eine stark abgerundete Form mit breitkonischem Unterteil und mit niedrigem Hals kennzeichnend. Das bruchstückhaft erhalten gebliebene Gefäß aus dem Grab 2.71 von Svodín kann man nur mit Vorbehalt in diese Typengruppe einordnen (Abb. 15d).

\subsubsection{Gattung 1c-doppelkonische Becher}

Die doppelkonischen Becher konnten in vier Serien, 5 Typengruppen und 18 Varianten gruppiert werden. Sie wurden nach ähnlichen Maßbezeichnungen klassifiziert wie die dreigliedrigen Becher (Abb. 16).

Unter den 42 untersuchten Bechern von doppelkonischer Form befinden sich 14, die aus Alsónyék stammen und die drei Typengruppen und nicht weniger als 15 Varianten untereinander bilden. Die Becher der Typengruppe $1 \mathrm{c} 2 \mathrm{~b}$ (mit acht Varianten) weisen einen niedrigeren, breitkonischen und nur leicht eingezogenen Unterteil auf, darauf stehen der betont kantige Bauchumbruch und der nach innen geschweifte Schulterteil. Der Hals ist kurz und zylindrisch mit ausladendem Rand. Diese Typengruppe tritt auch in Zengővárkony (Gräbergruppen 2a, 2b, $6 \mathrm{c}$ und 8b), Villánykövesd, Pári-Altacker, Mórágy und Veszprém auf (Abb. 17a-c). 


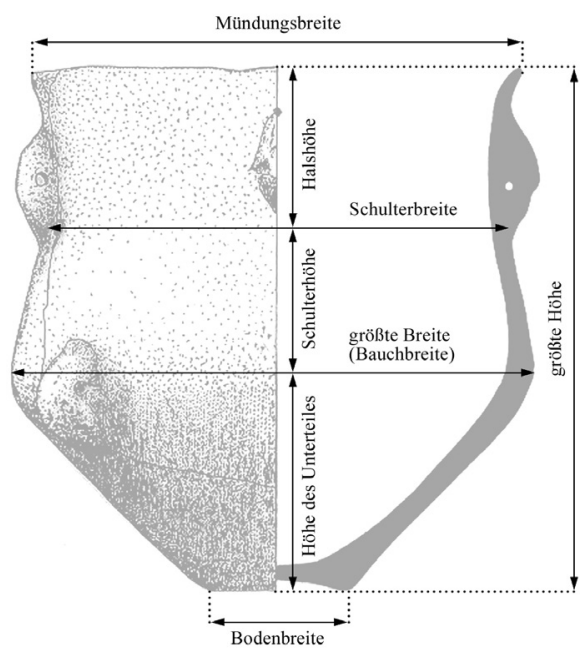

Abb. 16. Maßbezeichnungen eines doppelkonischen Bechers auf den verschiedenen Ebenen
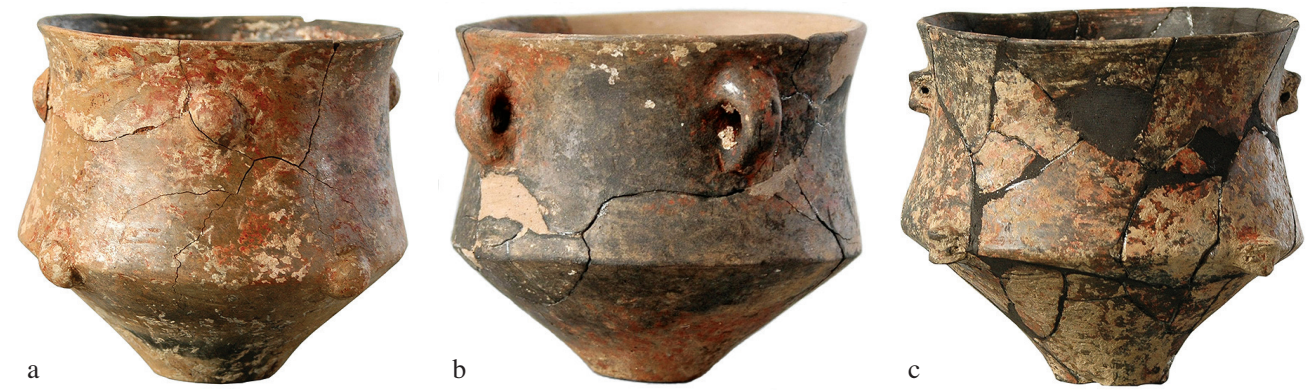

Abb. 17a-c. Alsónyék-Bátaszék. Doppelkonische Becher der Typengruppe 1c2b.

a: Variante 1c2b2, Grab an811; b: Variante 1c2b4, Grab an801; c: Variante 1c2b5, Grab an811
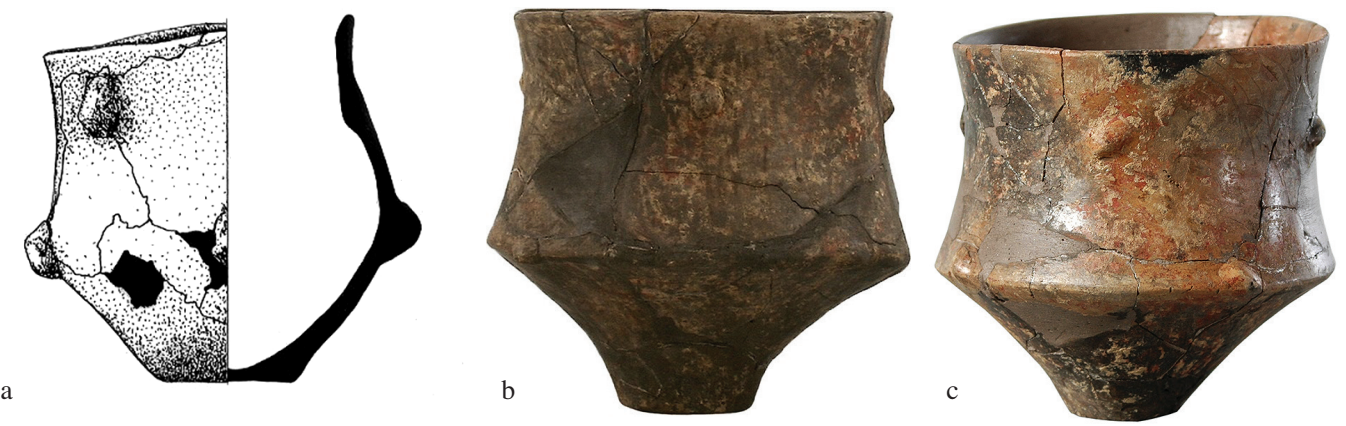

Abb. 18a-c. Alsónyék-Bátaszék. Doppelkonische Becher der Typengruppe 1c3a a: Variante 1c3a1, Grab an792; b: Variante 1c3a4, Grab az333; c: Variante 1c3a5, Grab an853

Das gemeinsame Charakteristikum der Keramiken der Typengruppe 1c3a (mit fünf Varianten) liegt in einer höheren Gestalt. Der Unterteil ist schlanker und lädt stärker nach innen aus, auch der obere Gefäßteil is länger ( $A b b$. $18 a-c)$. Ihre Varianten erscheinen noch in Zengővárkony (Gräbergruppen 5, 6d, 11-13), Mórágy und Veszprém.

Die Exemplare der Typengruppe 1c4a (mit zwei Varianten) besitzen eine noch höhere und schlankere Form. Auf dem niedrigeren, breitkonischen Unterteil steht der länger geformte obere Gefäßteil mit ausladendem Hals und der Bauchumbruch ist leicht kantig (Abb. 19a-b). Die Varianten dieser Typengruppe sind auch in Mórágy und Veszprém nachgewiesen. 

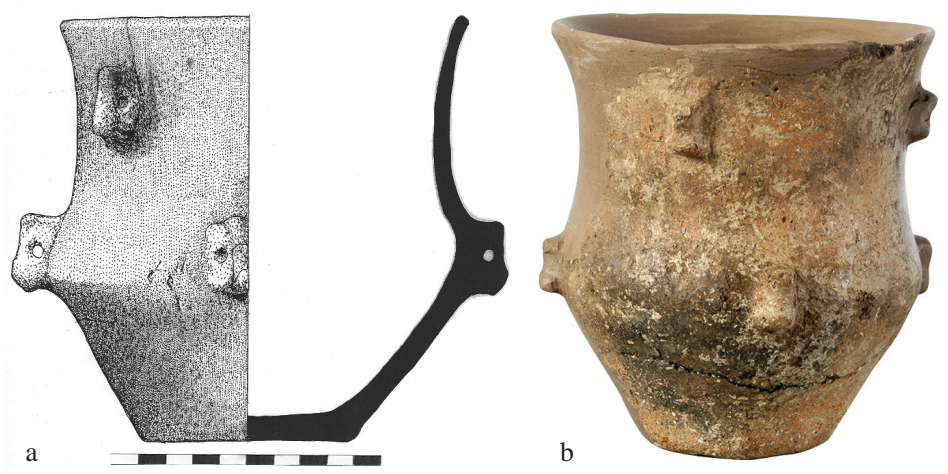

Abb. 19a-b. Alsónyék-Bátaszék. Doppelkonische Becher der Typengruppe 1c4a. a-b: Variante 1c4a1, Grab an1473 und Grab an850

\subsubsection{Gattung 1d-eingliedrige Becher}

Die „eingliedrigen“ Becher mit niedrigem Unter- und höherem Oberteil werden durch die Indizes und den Körperbau als eine selbstständige Gattung definiert. Nach den Indexwerten und den formalen Abweichungen bestimmen wir 2 Serien, 3 Typengruppen und 7 Varianten unter ihnen.

Unter 25 Exemplaren von kugeligen, eingliedrigen Bechern der untersuchten Lengyel-Nekropolen gibt es drei Exemplare von zwei Typengruppen, die aus Alsónyék stammen. Die Typengruppe 1d1b (mit fünf Varianten) ist unten konisch, die Wandung ist abgerundet und der Hals ist kurz und zylindrisch (Abb. 20a). Alle anderen Exemplare dieser Typengruppe stammen aus Mórágy. Für den Becher der Typengruppe 1d2a (mit fünf Varianten) sind profilierter Unterteil, betonte Bauchgegend und höherer, zylindrischer Hals typisch (Abb. 20b). Auch diese Typengruppe ist nur noch in Mórágy vertreten.
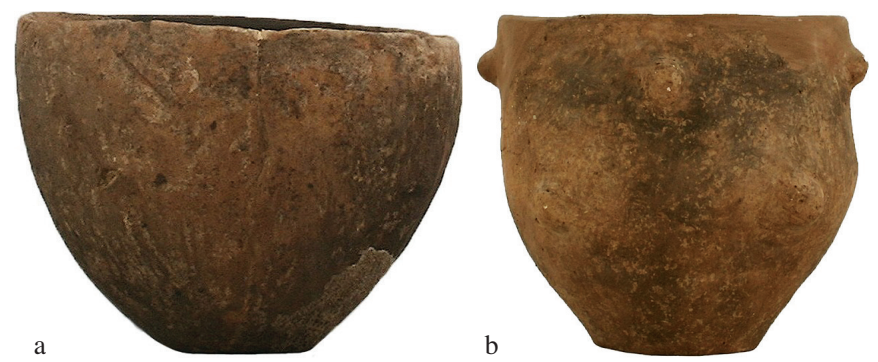

Abb. 20a-b. Alsónyék-Bátaszék. Kugelige und konische Becher der Typengruppen 1d1b und 1d2a1. a: Variante 1d1b5, Grab az263; b: Variante 1d2a1, Grab az673

\subsubsection{Gattung le-Näpfe}

Bereits in unseren früheren Arbeiten wurden die Gefäße zu den Näpfen gerechnet, deren Höhen-BreitenVerhältnis bei etwa 1:1 liegt und die größte Breite dieser zumeist konischen Keramikformen im Allgemeinen der Größe des Randdurchmessers entspricht. ${ }^{35} \mathrm{Im}$ Fall der Exemplare der Bechergattungen handelt es sich gewöhnlich um dünnwandige Keramiken aus fein geschlämmtem Ton. Bei der Gattung der Näpfe handelt es sich dagegen zumeist um aus grob gemagertem Ton hergestellte Gefäße von kugeliger oder profiliert konischer Form. Sie zeichnen sich durch eine große Formvariabilität aus. Sie wurden nach 4 Serien, 19 Typengruppen und 49 Varianten gruppiert.

\footnotetext{
${ }^{35}$ Strobel 1996, 22; Zalai-GaÁl 2007a, 53.
} 
In die Analyse der Grabkeramik der Lengyel-Gräberfelder im westlichen Karpatenbecken nahmen wir 104 Artefakte von Näpfen auf. Unter ihnen befinden sich lediglich 20, die aus Alsónyék stammen. Sie verteilen sich nach neun Typengruppen.

Die Typengruppe 1e1a (mit vier Varianten) kann mit breitkonischem Unterteil, mit leicht betontem Bauchumbruch und mit niedrigerem zylindrischem Hals charakterisiert werden (Abb. 21a). Ähnlich ausgeprägte Exemplare sind noch in Mórágy und in Svodín belegt. Die Typengruppe 1e1b der Näpfe mit hoher konischer Form erscheint allein in Alsónyék-Bátaszék (Abb. 21b).
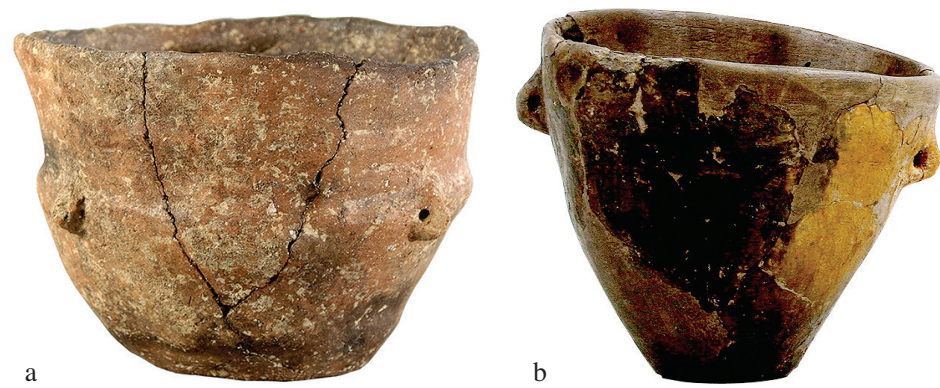

Abb. 21a-b. Alsónyék-Bátaszék. Konische Näpfe der Typengruppen 1e1a und 1e1b. a: Variante 1e1a3, Grab an829; b: Variante 1e1b1, Grab az1006

Das gemeinsame Charakteristikum der Näpfe der Typengruppe 1e2c (mit vier Varianten) liegt in der niedrigen doppelkonischen Körperform mit breitkonischem Unterteil. Zwischen den einzelnen Napftypen dieser Typengruppe sind gleichzeitig starke formale Abweichungen festzustellen (Abb. 22a-d). Mit einer Ausnahme (Mórágy) sind all diese Gefäße für das Gräberfeld von Alsónyék typisch.
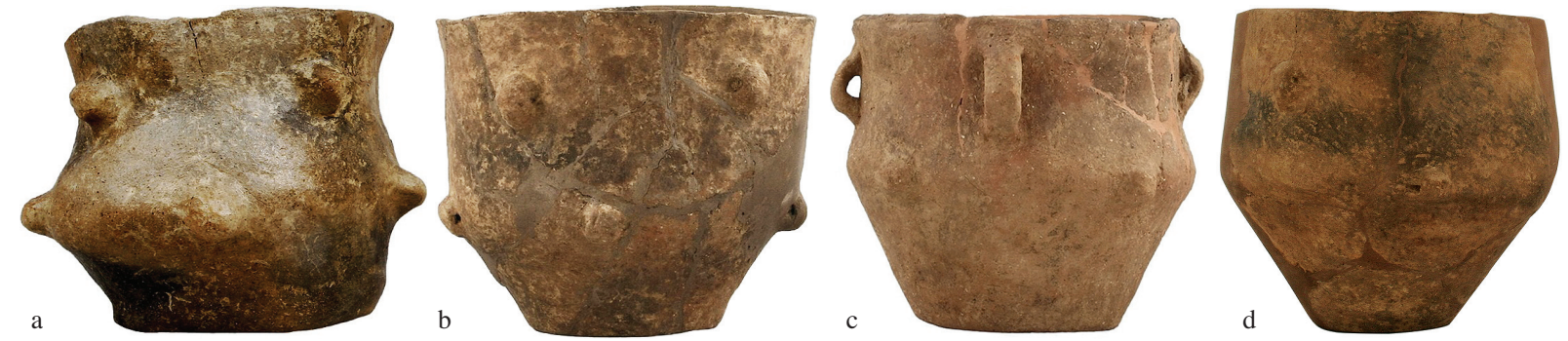

Abb. 22a-d. Alsónyék-Bátaszék. Konische Näpfe der Typengruppe 1e2c.

a: Variante 1e2c1, Grab az1967; b: Variante 1e2c2, Grab az743; c: Variante 1e2c3, Grab az319; d: Variante 1e2c4, Grab az1320

Die Näpfe der Typengruppe 1e2d (eine Variante) weisen eine ähnliche, aber am Bauch betont abgerundete Gestalt auf (Abb. 23a). Die Gefäße dieser typologischen Einheit können noch in Zengővárkony (Gräbergruppe 2b) und Mórágy nachgewiesen werden. Die Näpfe der Typengruppe 1a3a (mit drei Varianten) besitzen dagegen eine viel höhere und schlankere Form mit abgerundetem Bauch und kürzerem zylindrischem Hals (Abb. 23b-d). Parallelfunde stammen aus Zengővárkony (Gräbergruppe 6b), Mórágy und möglicherweise aus Svodín (Grab 6.72).

Die Näpfe der Typengruppe 1e3c treten in sechs Formvarianten auf. Sie können mit niedrigem und breitkonischem Unterteil, abgerundetem Bauch und hohem, zylindrischem Hals bezeichnet werden (Abb. 24a). Auch in Zengővárkony (Gräbergruppe 2b) und Svodín kommen ähnlich ausgeprägte Keramiken vor. Die Gefäße der Typengruppe 1e4b (mit drei Varianten) sind unten höher und konisch ausgeformt, der obere Gefäßteil ist dagegen länger $(A b b .24 b)$. Diese Typengruppe ist auch in Pécsvárad, Mórágy und Veszprém vertreten. Der Unterteil und die Bauchpartie der Näpfe der Typengruppe 1e4c (mit drei Varianten) sind breiter und abgerundeter ausgeprägt, der Hals ist kürzer und zylindrisch $(A b b .24 c)$. Die Typengruppe erscheint auch noch in Mórágy und Veszprém. 

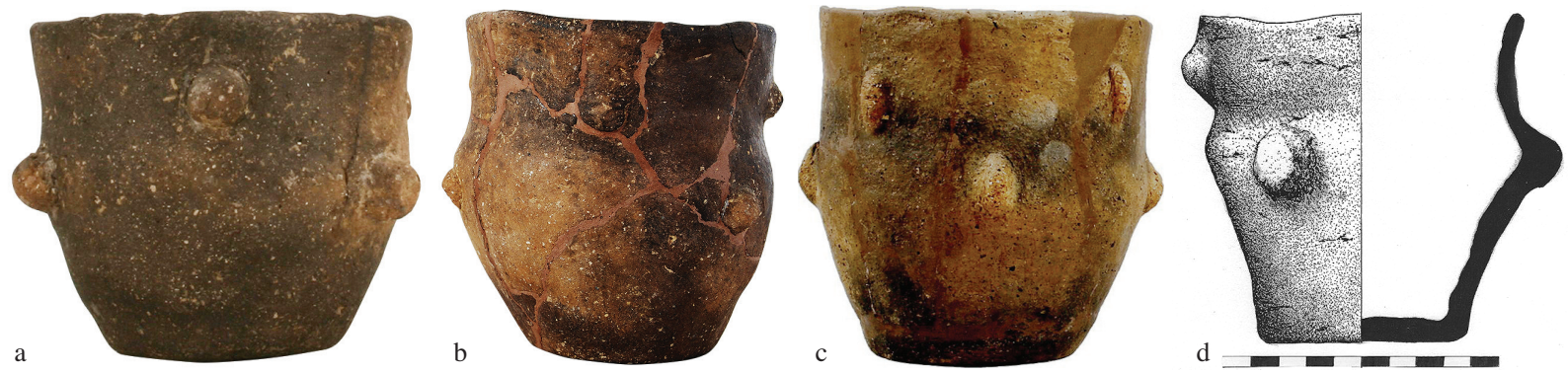

Abb. 23a-d. Alsónyék-Bátaszék. Zweigliedrige Näpfe der Typengruppe 1e3a.

a: Variante 1e2d1, Grab az1190; b-c: Variante 1e3a2, Grab an3020 und Grab az1391; d: Variante 1e3a3, Grab an3103
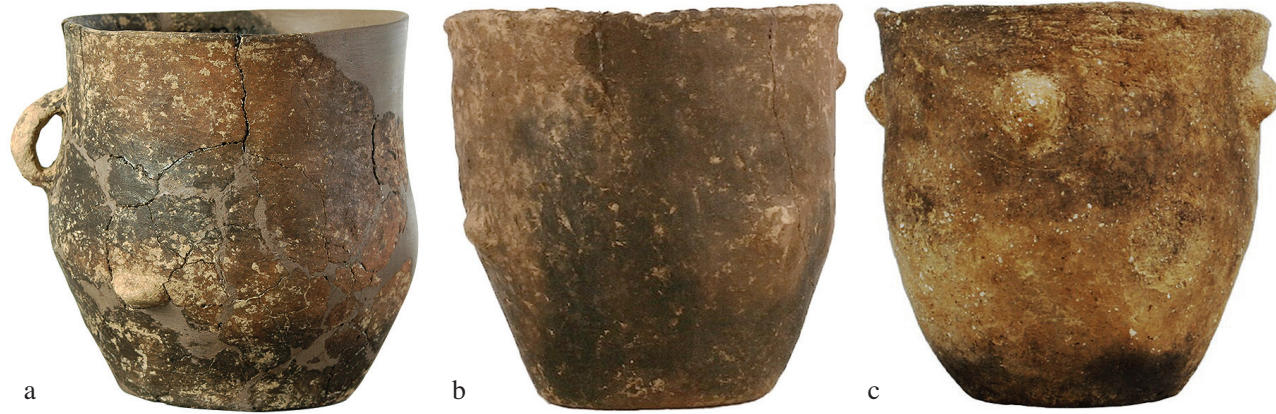

Abb. 24a-c. Alsónyék-Bátaszék. Kugelige und konische Näpfe der Typengruppen 1e3c, 1e4b und 1e4c a: Variante 1e3c5, Grab an825; b: Variante 1e4b2, Grab az304; c: Variante 1e4c1, Grab az2028

\subsection{Klasse 2 - Breitgefäße}

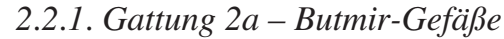

Das wichtigste Merkmal dieser in der Literatur als „Butmir-Gefäße“ oder als „Gefäße mit eingezogener Mündung“ angesprochenen Gattung ist der aus einem konischen Unterteil und einem gewölbten Oberteil (Schulter) mit im Randbereich einziehender Wandung bestehende Gefäßkörper. Der Längen-Breiten-Index dieser Keramiken liegt zwischen 0,57 und $1,02 .{ }^{36}$

Diese Gattung mit den untersuchten Gefäßen aus dem westlichen Karpatenbecken der Lengyel-Kultur umfasst 3 Serien, 15 Typengruppen und 35 Typen. Im keramischen Bestand der 113 Butmir-Gefäße findet man lediglich 34, die aus Alsónyék untersucht wurden. Sie können in fünf Typengruppen und 18 Typen eingereiht werden. Die bei der Analyse dieser Gefäße in Betracht gezogenen Maßbezeichnungen stellt Abb. 25 dar.

Die Gefäße der Typengruppe 2alb (mit drei Varianten) sind gedrungen, sie haben einen niedrigen, breitkonischen, sich nach innen gezogen ausbreitenden Unterteil, einen betonten Bauchumbruch sowie eine stark abgerundete Schulter (Abb. 26a-b). Ähnliche Gefäßformen sind lediglich in Mórágy bestätigt.

Die Exemplare der Typengruppe 2ale (mit fünf Varianten) sind ähnlich profiliert, sie besitzen dagegen eine höhere Körperform mit höherem Unterteil $(A b b .27 a-c)$. Diese typologische Einheit ist noch in Zengővárkony (Gräbergruppen 6c und 12), Mórágy und Veszprém vertreten.

Die Butmir-Gefäße der Typengruppe 2a2a (mit drei Varianten) können durch einen niedrigen und konischen Unterteil und eine stark abgerundete hohe Schulter charakterisiert werden. Die Variante 2a2a1 wurde auch mit einem kürzeren Hals versehen $(A b b .28 a-b)$. Die Exemplare dieser Typengruppe treten auch noch in Zengővárkony (Gräbergruppe 9), Villánykövesd, Mórágy und Veszprém auf.

\footnotetext{
${ }^{36}$ ZALAI-GAÁL 2007a, 57.
} 


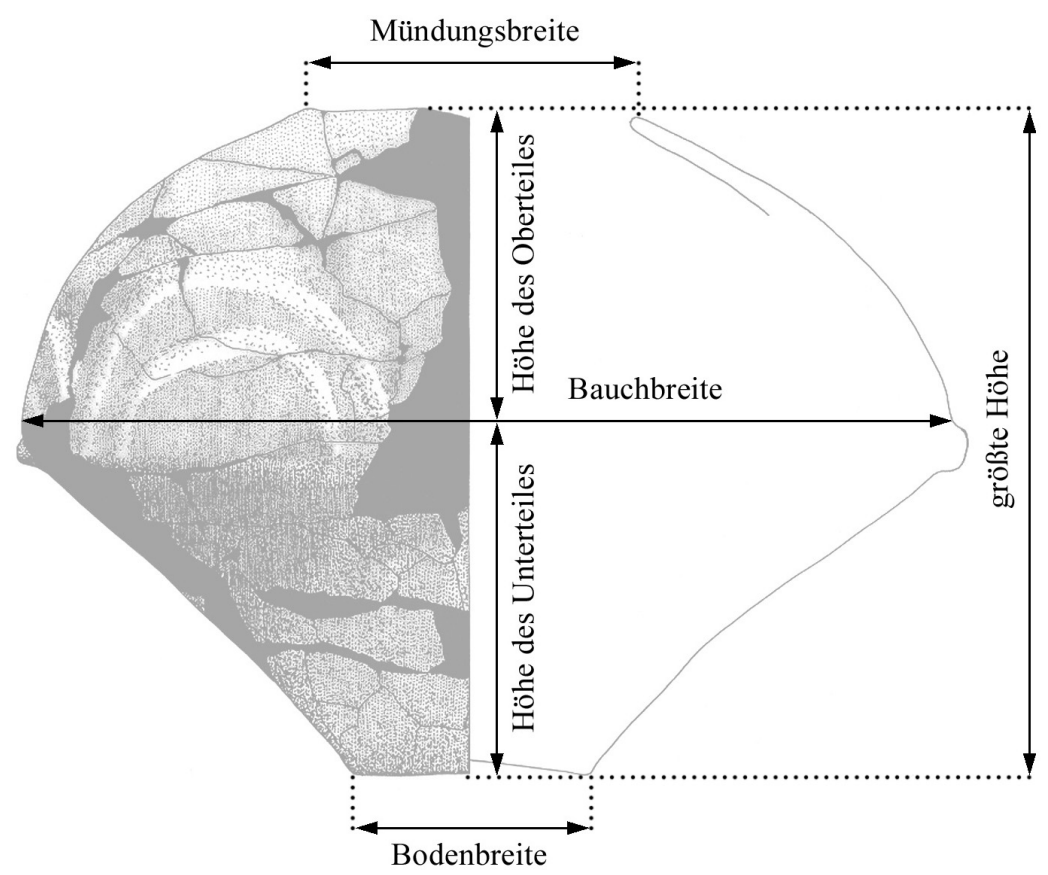

Abb. 25. Maßbezeichnungen eines Butmir-Gefäßes auf den verschiedenen Ebenen

Ein hoher, breitkonischer Unterteil und eine niedrigere Schulterpartie sowie ein kantig geformter Bauchumbruch sind für die Butmir-Gefäße der Typengruppe 2a2d (mit vier Varianten) typisch (Abb. 29a-c). Solche erscheinen auch in Zengővárkony (Gräbergruppen 2b, 6c bzw. 11) und Mórágy.

Die Exemplare der Typengruppe 2a2e (mit drei Varianten) besitzen einen hohen, sich nach innen geschweift breit werdenden Unterteil und einen viel niedrigeren, gedrungenen Oberteil mit kantig geformtem Bauchumbruch $(A b b .30 a-c)$. Ähnliche Keramiken kamen noch in Zengővárkony (Gräbergruppen 6d, 8b und 13), Villánykövesd, Mórágy und Veszprém zum Vorschein.
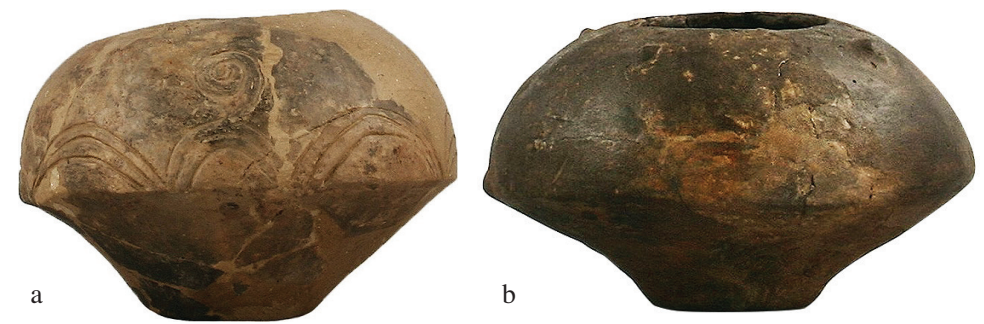

Abb. 26a-b. Alsónyék-Bátaszék. Butmir-Gefäße der Typengruppe 2a1b. a: Variante 2a1b1, Grab az228; b: Variante 2a1b2, Grab az1192
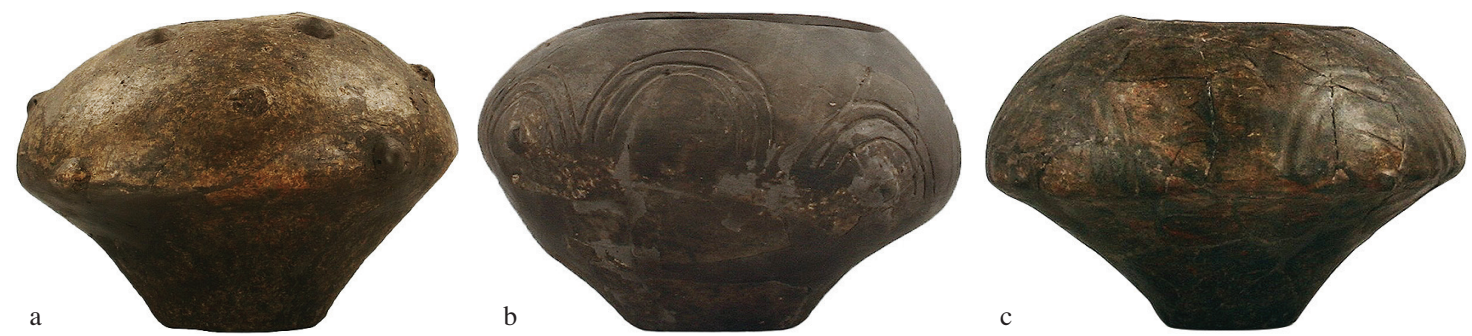

Abb. 27a-c. Alsónyék-Bátaszék. Butmir-Gefäße der Typengruppe 2a1e. a-b: Variante 2a1e5, Grab az335 und Grab az295; c: Variante 2a1e6, Grab az1006 
Ein hoher, breitkonisch geprägter Unterteil und eine extrem niedrige Schulterpartie sind für die Typengruppe 2a2f (mit zwei Varianten) charakteristisch $(A b b .31 a-b)$. Diese Typengruppe ist nur noch in Zengővárkony (Gräbergruppen 6d und 13) vorhanden.
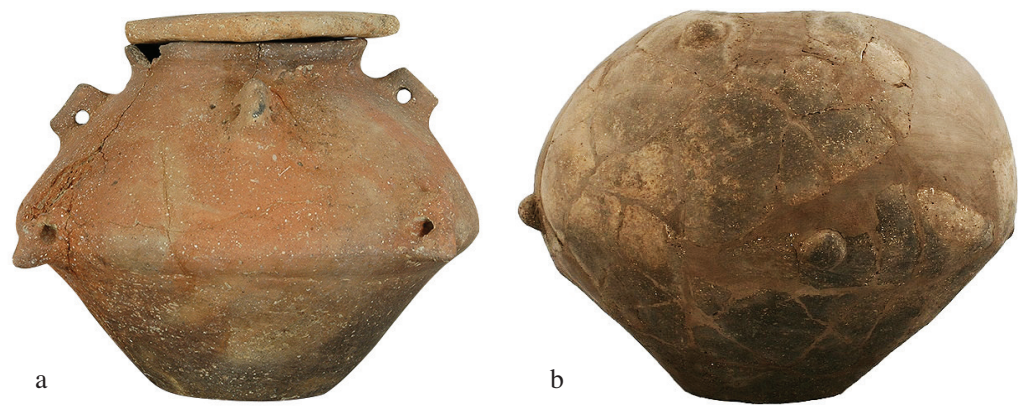

Abb. 28a-b. Alsónyék-Bátaszék. Butmir-Gefäße der Typengruppe 2a2a. a: Variante 2a2a1, Grab an1473; b: Variante 2a2a2, Grab az278
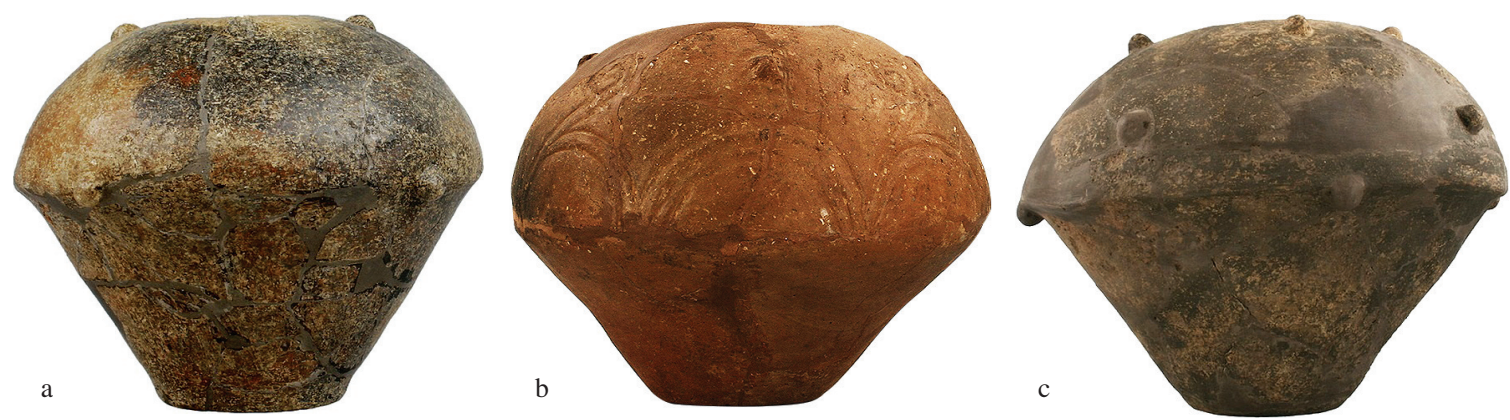

Abb. 29a-c. Alsónyék-Bátaszék. Butmir-Gefäße der Typengruppe 2a2d.

a: Variante 2a2d1, Grab az1967; b: Variante 2a2d2, Grab az324; c: Variante 2a2d4, Grab az319
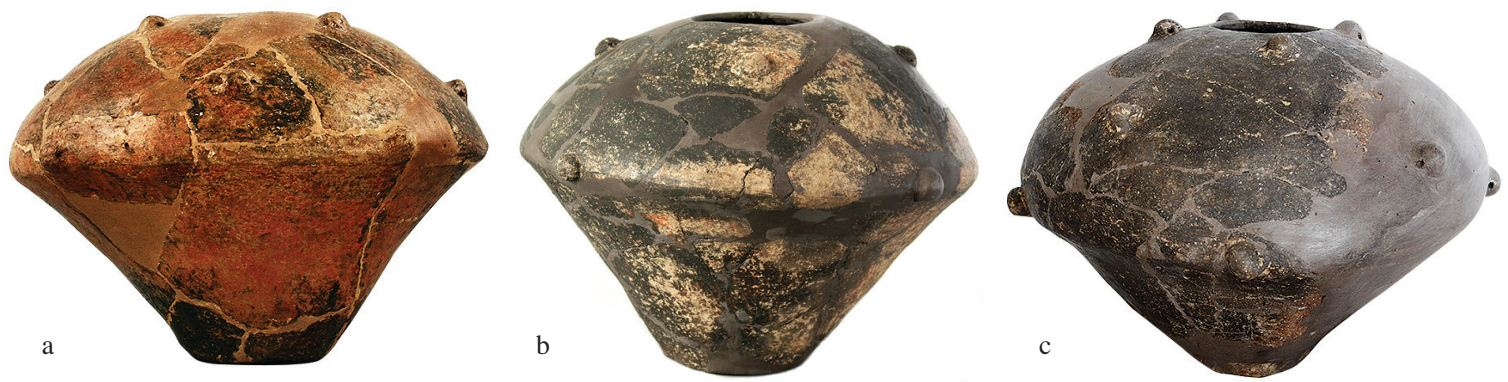

Abb. 30a-c. Alsónyék-Bátaszék. Butmir-Gefäße der Typengruppe 2a2e. a-b: Variante 2a2e1, Grab az1006 und Grab an769, c: Variante 2a2e2, Grab an1473
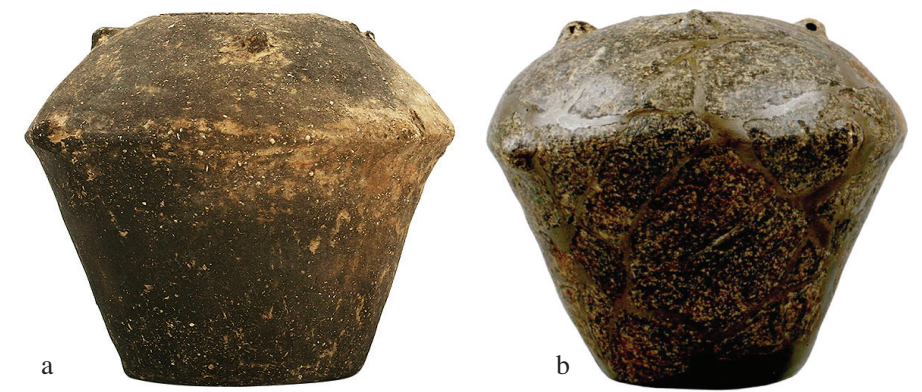

Abb. 31a-b. Alsónyék-Bátaszék. Butmir-Gefäße der Typengruppe 2a2f. a-b: Variante 2a2f2, Grab an5196 und Grab az2330 


\subsubsection{Gattung $2 b-$ Schüsseln}

Die größte Breite der Schüsseln entspricht etwa der doppelten Höhe. ${ }^{37}$ „Die Schwierigkeiten bei der Klassifikation und Gliedrung der Keramik, die auf den Umstand, dass innerhalb der neolithischen Gefäße keine Uniformität gegeben ist, zurückzuführen sind, zeigten sich bei der Typisierung von Schüsseln und Schalen.“"38

233 Exemplare wurden von den runden oder ovalen, profilierten oder konischen Schüsseln in die Analyse aufgenommen; aus Alsónyék stammen 38 Exemplare. Die verschiedenen Schüsselformen bilden 5 Serien, 26 Typengruppen und 61 Varianten untereinander. In Alsónyék treten aber nicht mehr als neun Typengruppen und 31 Varianten der Schüsseln auf. Die Maßbezeichnungen für die Klassifizierung der Exemplare dieser Gattung sind auf Abb. 32 vorgezeigt.

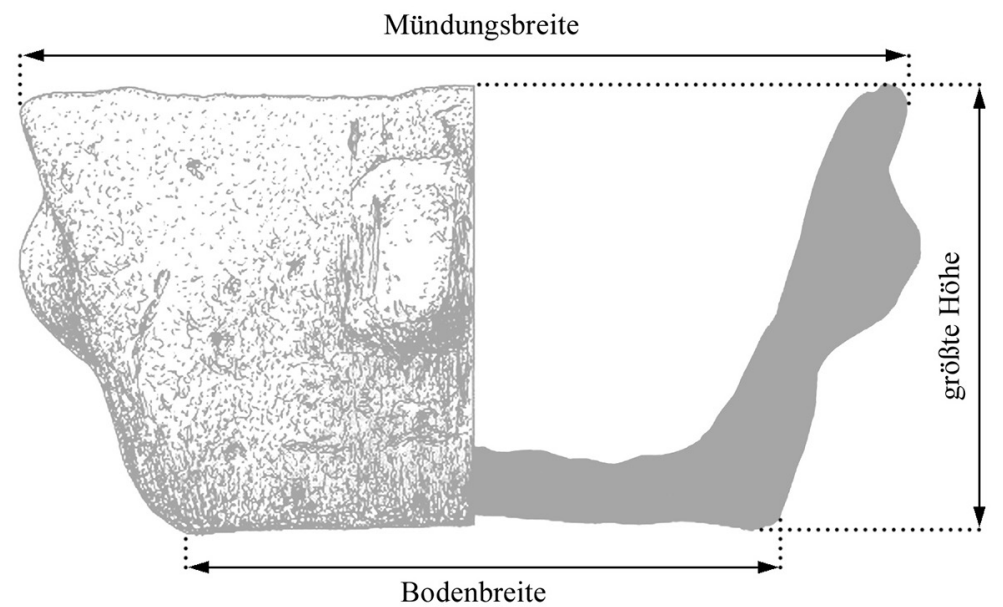

Abb. 32. Maßbezeichnungen von Schüsseln und Schalen auf den verschiedenen Ebenen

Die runden Schüsseln der Typengruppe $2 \mathrm{~b} 2 \mathrm{~b}$ (mit drei Varianten) weisen flache und sich nach oben breit werdende profilierte Körperform mit ausladendem Hals auf. Der Bauchumbruch ist leicht betont (Abb. 33a-b). Diese Gefäßform erscheint auch in Zengővárkony (Gräbergruppen 6c, 6d und 9).
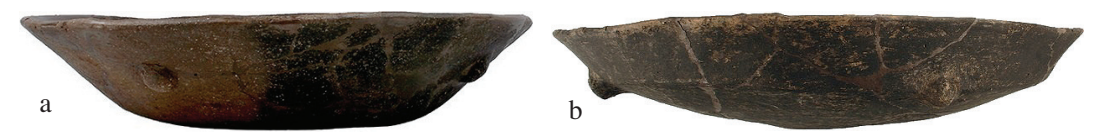

Abb. 33a-b. Alsónyék-Bátaszék. Schüsseln der Typengruppe 2b1b. a: Variante 2b1b2, Grab az2330; b: Variante 2b1b3, Grab az673

Die Typengruppe 2b2a (eine Variante) ist durch einen höheren Körper und einen sich ein wenig nach innen geschweift ausladenden Oberteil zu charakterisieren (Abb. 34). Die Exemplare dieser typologischen Einheit wurden auch in Zengővárkony (Gräbergruppe $2 b$ ) und Mórágy nachgewiesen.

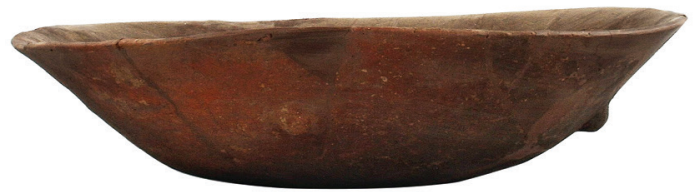

Abb. 34. Alsónyék-Bátaszék. Schüssel der Typengruppe 2b2a, Variante 2b2a1, Grab az293 

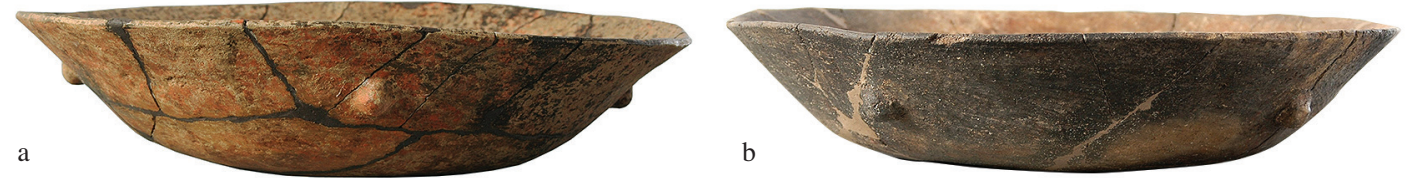

Abb. 35a-b. Alsónyék-Bátaszék. Schüsseln der Typengruppe 2b2c. a: Variante 2b2c2, Grab an769; b: Variante 2b2c3, Grab an801

Die Schüsseln der Typengruppe 2b2c (mit drei Varianten) wurden ähnlich profiliert als die der vorigen Typengruppe $(A b b .35 a-b)$. Der Unterschied zwischen ihnen besteht in den Maßbezeichnungen. Parallelfunde dieser Typengruppe wurden in Zengővárkony (Gräbergruppen 6a, 6b bzw. 13) und in Mórágy geborgen.

Der Typengruppe $2 \mathrm{~b} 2 \mathrm{e}$ (mit drei Varianten) teilten wir niedrige Schüsseln mit einfachem konischem Körper zu $(A b b .36 a-b)$. Solche treten noch in Zengővárkony (Gräbergruppe 6c), Mórágy und eventuell in Svodín auf.
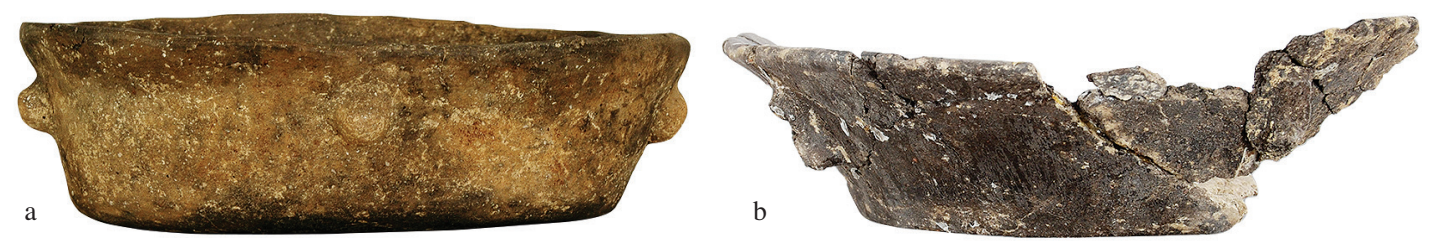

Abb. 36a-b. Alsónyék-Bátaszék. Schüsseln der Typengruppe 2b2e. a: Variante 2b2e1, Grab an853; b: Variante 2b2e2, Grab an1473

Die Schüsseln der Typengruppe 2b3a (mit fünf Varianten) sind ebenso konisch ausgestaltet, sie besitzen dagegen eine höhere Körperform ( $A b b .37 a-c)$. Die Typengruppe ist auch in Zengővárkony (Gräbergruppen 3b und 9), Mórágy und Svodín bestätigt.
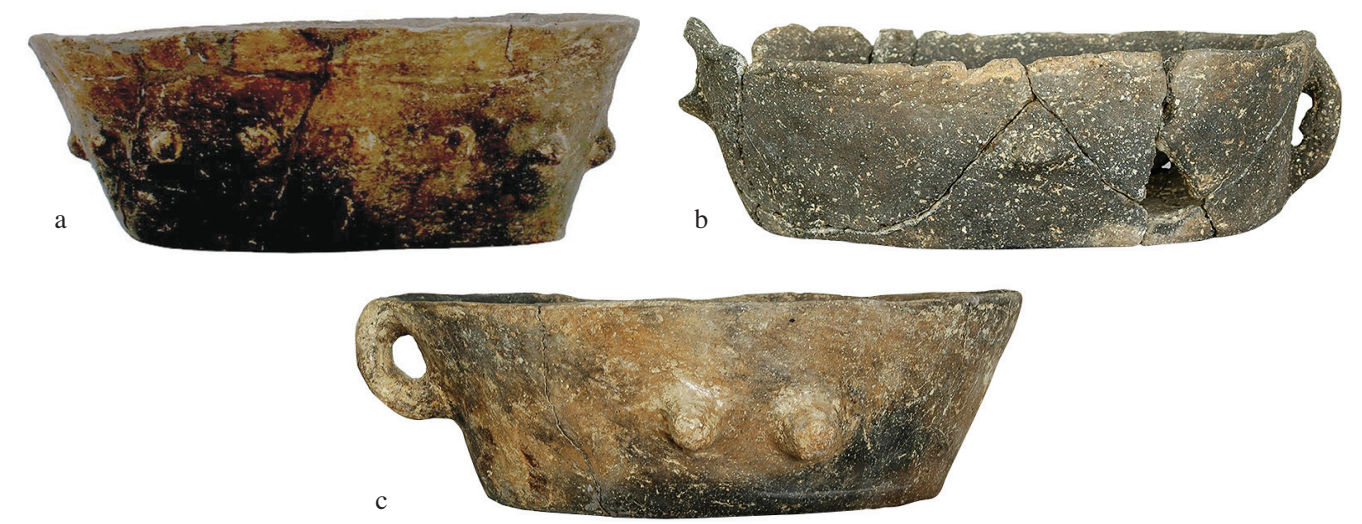

Abb. 37a-c. Alsónyék-Bátaszék. Schüsseln der Typengruppe 2b3a.

a: Variante 2b3a2, Grab az3131; b-c: Variante 2b3a3, Grab an3089 und Grab an963

Die der Typengruppe 2b3b (mit vier Varianten) zugeordneten Schüsseln haben eine hohe, konische Form mit breit werdendem Oberteil ( $A b b .38 a$ ), während die Exemplare der Typengruppe 2b3d (mit zwei Varianten) mit einem höheren und unprofilierten Körper ausgestaltet wurden $(A b b .38 b)$. Die erste Typengruppe kommt auch in Zengővárkony (Gräbergruppen 1, 2b, 6c, 6d bzw. 9) und Mórágy vor; die zweite wurde auch in Zengővárkony (Gräbergruppen 6c, 8b bzw. 12) und Villánykövesd belegt.

Die Formvarianten der Schüsseln der Typengruppe 2b4a (mit neun Varianten) zeigen eine schmalere und höhere Form mit kurzer Standfläche auf (Abb. 39a-d). Die Varianten dieser Typengruppe sind auch in Zengővárkony (Gräbergruppen 2b, 3a, 6c, 6d und 9), Mórágy, Pári-Altacker und Szekszárd-Ágostonpuszta vorhanden. 

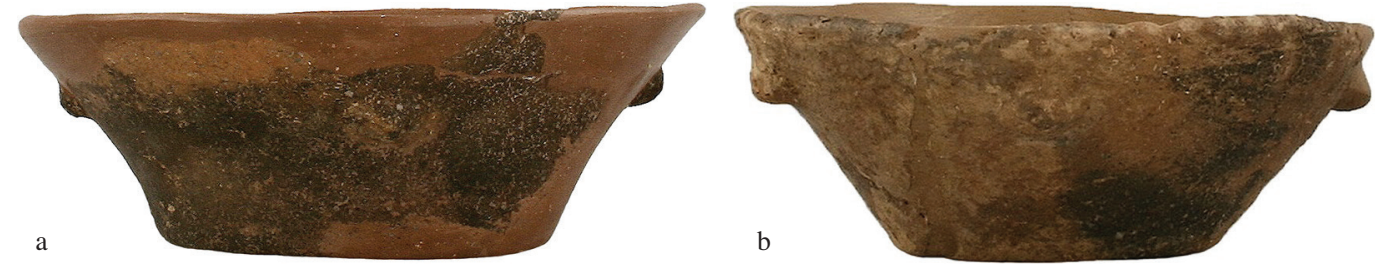

Abb. 38a-b. Alsónyék-Bátaszék. Schüsseln der Typengruppen 2b3b und 2b3d. a: Variante 2b3b2, Grab az190; b: Variante 2b3d2, Grab az318
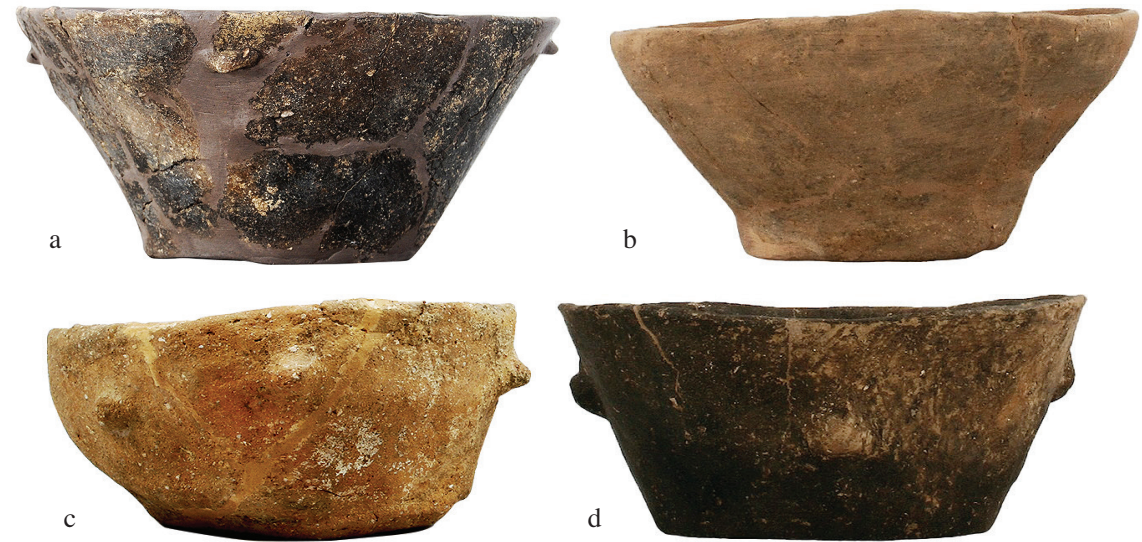

Abb. 39a-d. Alsónyék-Bátaszék. Formvarianten der Schüsseln der Typengruppe 2b4a.

a: Variante 2b4a4, Grab an426; b: Variante 2b4a6, Grab az272; c: Variante 2b4a7, Grab az304; d: Variante 2b4a8, Grab az304

Die Typengruppe 2b5b ist nur durch eine Variante und nur eine Keramik von Alsónyék vertreten. Sie zeigt eine doppelkonische Form mit niedrigem, breitkonischem Unterteil sowie mit einem kantigen Bauch und einem höheren zylindrischen Hals auf ( $A b b .40)$.

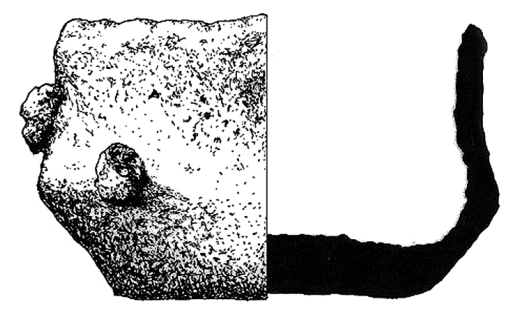

Abb. 40. Alsónyék-Bátaszék. Schüssel der Typengruppe 2b5b, Variante 2b5b1, Grab an3791

\subsubsection{Gattung $2 c-$ Schalen}

Die Gattung der Schalen war in unserer früheren Analyse über die südtransdanubische Lengyel-Grabkeramik durch ein-, zwei- und dreigliedrige Keramikgefäße vertreten, bei denen das Verhältnis zwischen Höhe und größte Breite 0,44-0,70 beträgt. In dem vorliegenden neuen analytischen System ordneten wir sie 2 Serien, 10 Typengruppen und 24 Varianten zu. Unter den 40 untersuchten Schalen befinden sich nur sechs, die auch in Alsónyék geborgen wurden. Sie bilden nicht mehr als drei Typengruppen und neun Varianten untereinander.

Die runde Schale der Typengruppe 2c1d (mit drei Varianten) besitzt einen breitkonischen und leicht profilierten Unterteil. Der Bauch ist ein wenig kantig modelliert und der Oberteil leicht profiliert bzw. zylindrisch ausgeprägt $(A b b .41)$. Diese Typengruppe wurde nur noch in Mórágy nachgewiesen. 


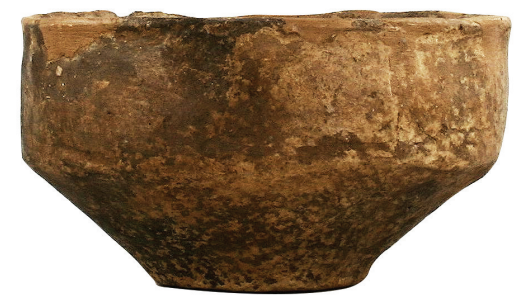

Abb. 41. Alsónyék-Bátaszék. Schale der Typengruppe 2c1d, Variante 2c1d3, Grab az724

Der Typengruppe 2c2d (mit fünf Varianten) werden doppelkonische Schalen mit sich nach innen gezogen breit werdendem Unterteil, mit kantig geformtem Bauchumbruch und höherem zylindrischem Hals zugeordnet. Man findet Varianten von schmalerer und breiterer Körperformen unter ihnen (Abb. 42a-b). Diese Typengruppe ist noch in Zengővárkony (Gräbergruppe 9), Villánykövesd, Pécsvárad und Mórágy vorhanden.
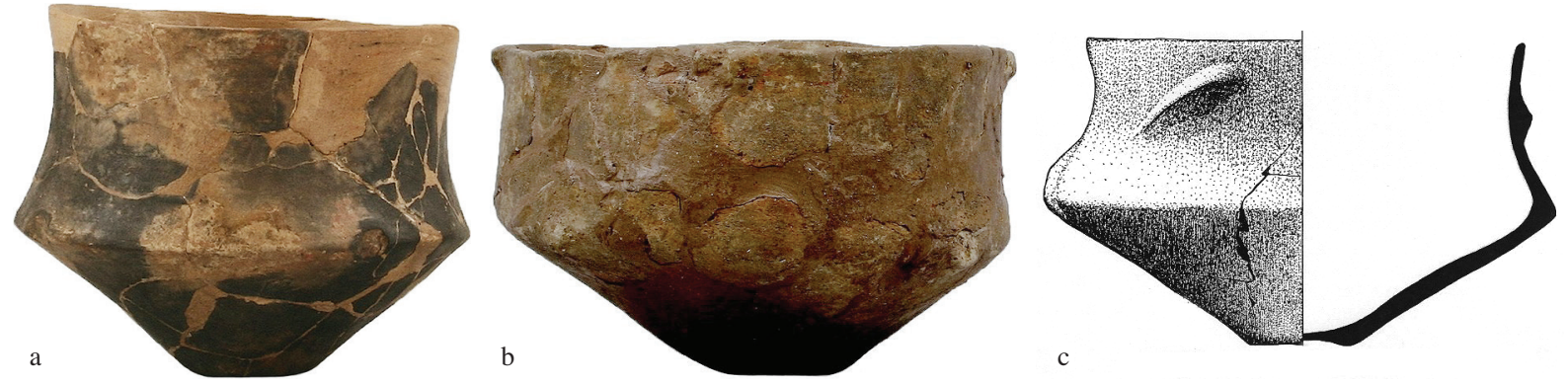

Abb. 42a-c. Alsónyék-Bátaszék. Schalen der Typengruppen 2c2d und 2c2e. a: Variante 2c2d2, Grab az333; b: Variante 2c2d4, Grab az3132; c: Variante 2c2e1, Grab an795

Nur ein Exemplar von Alsónyék vertritt die Typengruppe 2c2e. Für sie sind ein niedriger, breitkonischer und nach innen profilierter Unterteil, eine kantig betonte Bauch- und Schulterpartie sowie ein kurzer zylindrischer Hals kennzeichnend ( $A b b .42 c)$.

\section{DAS VORKOMMEN DER TYPOLOGISCHEN EINHEITEN DER GRABKERAMIK NACH REGIONEN/FUNDORTEN DER LENGYEL-KULTUR}

\subsection{Vorkommen und Verteilung der Serien}

Die vorgestellten lengyelzeitlichen Grabgefäße aus dem westlichen Karpatenbecken klassifizierten wir also nach 8 Gattungen, 29 Serien, 146 Typengruppen und 384 Varianten (Typen) mit Hilfe der Merkmalanalyse.

Die Grundformen der untersuchten Keramiken werden in Gattungen und Serien umfasst. Über Vorkommen und Verteilung der behandelten Serien kann Folgendes auf der Basis ihrer geographischen Situation resümiert werden. (Das Gräberfeld von Alsónyék wird als ein getrenntes Element untersucht.) Die Daten werden zahlenmäßig in Diagramm 5 zusammengefasst. Auf die Gefäßzahlen nach Fundort werden in eckigen Klammern hingedeutet.

a) Allein die Serie 2a3 der Butmir-Gefäße (3,45\%) wird nur in Südtransdanubien und Svodín vertreten [9-11].

b) Sowohl in dem Fundbestand der südtransdanubischen Altgrabungen als auch in Alsónyék treten fünf Serien $(17,24 \%)$ auf, die anderswo nicht belegt sind: Serie 1a4 von Fußgefäßen [17-3], 1c1 [3-1] von doppelkonischen Bechern, 1d1 [15-1] und 1d2 [7-2] von kugeligen Bechern sowie Serie 2b1 [6-3] von Schüsseln.

c) Es gibt sechs weitere Serien (20,69\%), also die Serien 1a1 [9-7-1] von Fußgefäßen, 1e1 von Näpfen [2-2-2], 2b4 [25-5-5] und 2b5 [4-1-2] von Schüsseln bzw. die Serien 2c1 [17-1-3] und 2c2 [20-5-2] von Schalen, die in den Altgrabungen und Alsónyék sowie in Svodín nachzuweisen sind. 
d) Die Serie 1 b4 von Schüsseln ist als die einzige (3,45 \%) zu behandeln, die sowohl in den Altgrabungen, Alsónyék und Svodín als auch in Veszprém und Friebritz belegt ist [58-19-17-3-1].

e) Die Becher der Serie 1b1 (3,45 \%) treten in den Altgrabungen, Svodin bzw. Lužianky auf [21-11-13].

f) Fünf Serien (13,79\%) erscheinen im Material der Altgrabungen, in Alsónyék, Svodín und Lužianky. Es handelt sich hierbei um die Serie 1b2 von Bechern [39-2-26-21], die Serien 1e2 [15-8-19-5] und 1e3 [8-5-23-1] von Näpfen und die Serien 2b2 [21-9-16-1] bzw. 2 b3 [35-18-18-9 ] von Schüsseln.

g) Zwei andere Serien (6,90\%) sind nur aus den Altgrabungen, in Alsónyék, Svodin und Lužianky wie auch in Veszprém und Friebritz bekannt. Hierzu gehören die Serie 1a2 von Fußgefäßen [30-11-10-2-2-1] und die Serie 1 b5 [18-11-15-8-1-3] von Bechern.

h) In den Altgrabungen, in Alsónyék und Veszprém (13,79\%, n=4) sind die Serien 1c2 [11-6-3], 1c3 [8-5-1] und 1c4 [1-2-1] von Schalen sowie die Serie 2a1 [25-10-2] von Butmir-Gefäßen bestätigt.

i) Die Serie $1 \mathrm{~b} 3$ von Schüsseln ist die einzige $(3,45 \%)$, die in den Altgrabungen, Alsónyék und Svodín sowie auch in Friebritz befindlich ist [33-13-36-2].

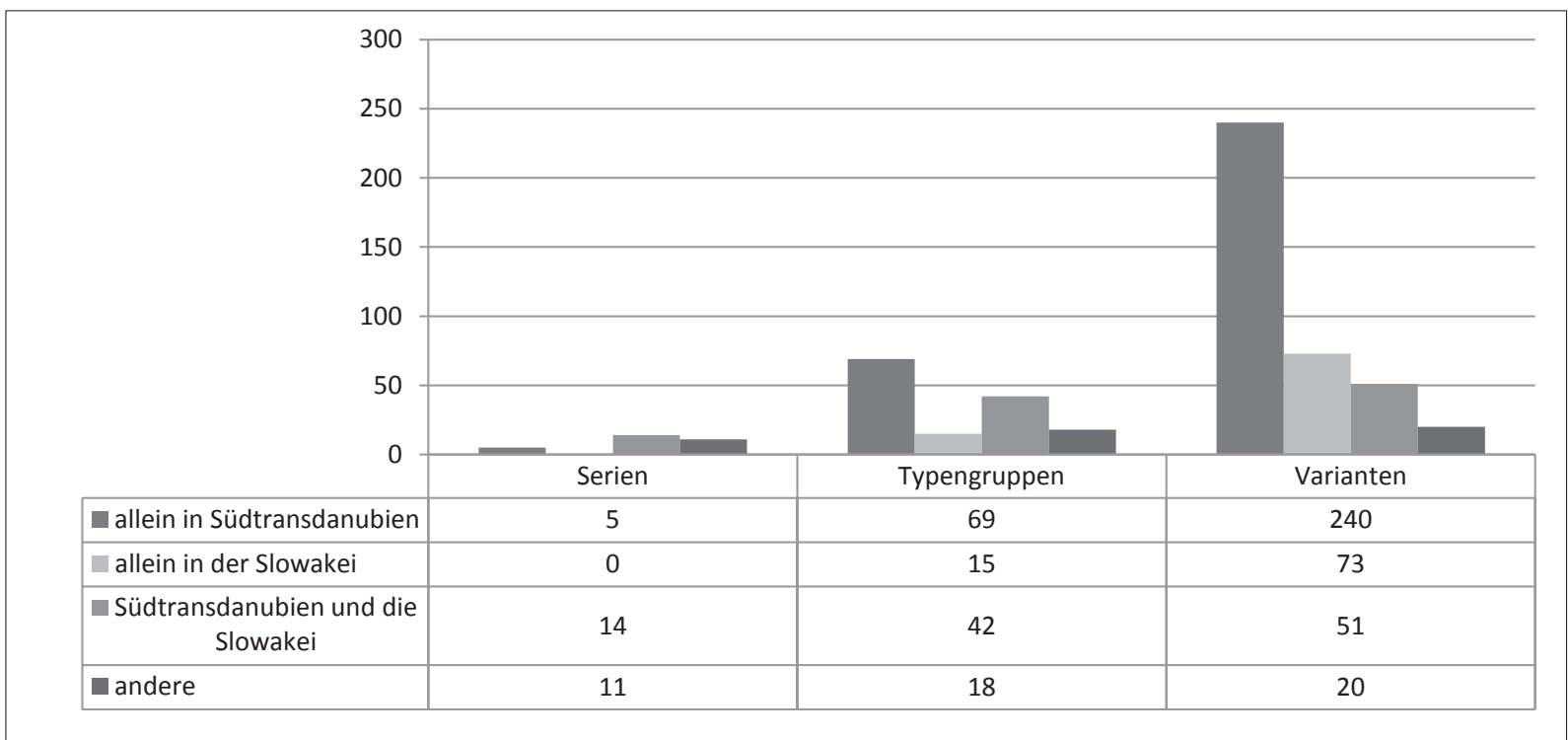

Diagramm 5. Vorkommen der typologischen Einheiten nach geographischen Regionen des Lengyel-Kreises

j) Die Serie 1a3 von Fußgefäßen stammt aus den Altgrabungen, Alsónyék, Svodín und Veszprém [52-6-15-1].

k) Die Napfserie 1e4 ist für die Gräberfelder der Altgrabungen, von Alsónyék, Lužianky und Veszprém typisch [8-5-3-3].

1) Die Serie 2a2 von Butmir-Gefäßen wird in den Altgrabungen, in Alsónyék, Svodín, Lužianky und Veszprém vertreten [28-24-2-1-2].

m) Die Serie 2a3 von Butmir-Gefäßen ist die einzige, die sowohl im Bestand der Altgrabungen als auch in Svodín vorkommt [9-11].

In größter Zahl sind also Serien bestätigt, die in sechs Regionen/Fundorten des Lengyel-Kreises beweisbar sind. Fünf andere Serien treten allein im südtransdanubischen Verbreitungsgebiet der Lengyel-Kultur auf und die Zahl der in vier Regionen/Fundorten nachweisbaren Serien beträgt je zwei (Südtransdanubien, Svodín, Lužianky bzw. Südtransdanubien und Veszprém). Beachtenswert ist auch, dass beinahe die Hälfte, d. h. 44,83 \% (13), der untersuchten keramischen Serien nur in den südtransdanubischen und südslowakischen Regionen/Fundorten der Lengyel-Kultur im westlichen Karpatenbecken erscheint. 


\subsection{Vorkommen und Verteilung der Typengruppen}

Die typologischen Merkmale der Keramikgefäße zeigen innerhalb der einzelnen Typengruppen eine wesentlich größere Ähnlichkeit zueinander als im Fall der Serien. Bei den Typengruppen kann eine viel größere Streuung nach Regionen/Fundorten erfahren werden als bei den Serien. Das Vorkommen und die Verteilung der 146 definierten Typengruppen der Lengyel-Keramik aus dem westlichen Karpatenbecken stellen ein aus 18 Elementen bestehendes Bild dar (Diagramm 5).

a) Der Anteil der allein in den Altgrabungen nachgewiesenen Typengruppen beträgt 21,53\% $(\mathrm{n}=31) .{ }^{39}$ $(\mathrm{n}=7) .^{40}$

b) Die nur in Alsónyék vorhandenen Typengruppen werden durch einen Prozentsatz von 4,06\% vertreten

c) $5,56 \%(\mathrm{n}=9)$ der Typengruppen erscheint allein in Svodin..$^{41}$

ein. $^{42}$

d) Der Anteil der nur in Lužianky belegten keramischen Typengruppen nimmt nicht mehr als 3,47 \% $(\mathrm{n}=5)$

e) Die Typengruppen 1a2h von Fußgefäßen und 1c2a von doppelkonischen Bechern sind allein für die Gräbergruppe von Veszprém typisch $(1,39 \%, \mathrm{n}=2)$.

f) Es gibt keine, allein in Friebritz vorhandene Typengruppe.

tigt. $^{43}$

g) 2,78\% $(\mathrm{n}=4)$ der Typengruppen wurde im Material der Altgrabungen, in Svodín sowie Friebritz bestä-

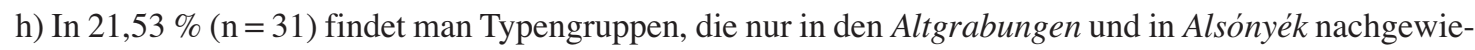
sen werden können. ${ }^{44}$

i) Mit einem Prozentsatz von 4,17\% $(\mathrm{n}=6)$ treten Typengruppen auf, die nur die Altgrabungen und Alsónyék bzw. Svodín charakterisieren. ${ }^{45}$

j) $16,67 \%(n=24)$ der Typengruppen erscheint nur im Bestand der Altgrabungen und in Svodin. ${ }^{46}$

k) Der Anteil der nur in den Altgrabungen und Alsónyék sowie in Veszprém auftretenden Typengruppen macht $6,94 \%(n=10)$ aus. $^{47}$

1) Die Typengruppe 1a3d [1-7-1] der Fußgefäße ist die einzige, die nur aus den Altgrabungen, Svodín und Veszprém bekannt ist.

m) 2,08 \% (n=3) der Typengruppen kann allein in den Altgrabungen und Lužianky bestätigt werden. ${ }^{48}$

n) 6,25\% (n=9) der Typengruppen ist für die Altgrabungen, Svodín und Lužianky charakteristisch. ${ }^{49}$

o) Die Typengruppe 2b2f der Schüsseln [5-1] erscheint nur in Svodín und Lužianky.

${ }^{39}$ Typengruppen 1a1b, 1a2f, 1a2g, 1a3c, 1a3f, 1a3g, 1a3h, $1 \mathrm{a} 4 \mathrm{~b}$ und 1a4d von Fußgefäßen, 1b2c, 1b3d, 1b4b, 1b4d und 1b5e von dreigliedrigen Bechern, 1d1a von kugeligen Bechern, 1e2g, 1e4d und 1e4e von Näpfen, 2a1a, 2a1c und 2a1d von Butmir-Gefäßen, 2b2b, $2 \mathrm{~b} 3 \mathrm{e}, 2 \mathrm{~b} 5 \mathrm{a}, 2 \mathrm{~b} 5 \mathrm{c}$ und $2 \mathrm{~b} 5 \mathrm{~d}$ von Schüsseln, 2c1b, 2c1c, $2 \mathrm{c} 2 \mathrm{a}, 2 \mathrm{c} 2 \mathrm{c}$ und $2 \mathrm{c} 2 \mathrm{f}$ von Schalen.

${ }^{40}$ Typengruppen 1a3i und 1a4a von Fußgefäßen, 1b5i und 1b5j von dreigliedrigen Bechern, 1e1b von Näpfen, 2b5b von Schüsseln und 2c2e von Schalen.

${ }^{41}$ Typengruppen 1a2a von Fußgefäßen, 1b1d, 1b5a und $1 b 5 f$ von dreigliedrigen Bechern, 1e4a von Näpfen, 2b3c, 2b4b, 2b5e und $2 \mathrm{~b} 5 \mathrm{f}$ von Schüsseln.

${ }^{42}$ Typengruppen $1 \mathrm{a} 2 \mathrm{~b}$ von Fußgefäßen, 1b1a von dreigliedrigen Bechern, 1e2a von Näpfen, $2 \mathrm{a} 2 \mathrm{~g}$ von Butmir-Gefäßen und 2b3f von Schüsseln.

${ }^{43}$ Typengruppen 1a2c von Fußgefäßen [2-1-1], 1b3a [210-2], 1b4a [3-12-1] und 1b5c [3-14-1] von Bechern.

${ }^{44}$ Typengruppen 1a2e [1-1], 1a2k [7-4], 1a21 [5-5], 1a3e [16-1], 1a3k [7-3] und 1a4c [9-1] von Fußgefäßen, 1b1f [4-1], 1b2f [8-2], 1b3c [9-4], 1b3e [11-9], 1b4f [11-4], 1b4g [1-1], 1b4h [1-2], $1 \mathrm{~b} 5 \mathrm{k}$ [1-2] und 1b51 [2-1] von dreigliedrigen Bechern, 1c1a [3-1] von doppelkonischen Bechern, 1d1b [10-1] und 1d2a [7-2] von kugeligen Bechern, 1e2c [1-7] und 1e2d [3-1] von Näpfen, 2a1b [4-2], 2a2d [7-9] und 2a2f [2-4] von Butmir-Gefäßen, 2b1b [5-3], 2b2a [2-1], 2b2c [8-4], 2b3b [9-4], 2b3d [4-4] und 2b4a [22-5] von Schüsseln, 2c1d [7-1] und 2c2d [12-3] von Schalen.

45 Typengruppen 1a1a von Fußgefäßen [7-7-1], 2b2e [5-42] und 2b3a [7-10-4] von Schüsseln, 1e1a [2-1-2], 1e3a [2-4-1] und $1 \mathrm{e} 3 \mathrm{c}$ [4-1-7] von Näpfen.

46 Typengruppen 1a2d [1-4], 1a2i [1-3], 1a3a [1-3], 1a3b [7-2] und 1a3j [1-3] von Fußgefäßen, 1b1c [12-2], 1b3b [11-24], 1b4c [8-4], 1b4e [13-1] und 1b5b [8-2] von dreigliedrigen Bechern, 1e2b [1-6], 1e2e [1-1] und 1e2h [1-2] von Näpfen, 2a2b [1-1], 2a2c [4-1], 2a3a [2-3], 2a3b [2-1] und 2a3c [5-7] von Butmir-Gefäßen, 2b2d [49], 2b3h [5-7], 2b3i [6-4] und 2b4c [3-1] von Schüsseln, 2c1a [2-3] und $2 \mathrm{c} 2 \mathrm{~b}[2 \mathrm{c} 2 \mathrm{~b}]$ von Schalen.

47 Typengruppen 1a2j von Fußgefäßen [6-2-1], 1b4i [1312-3] von dreigliedrigen Bechern, 1c2b [11-6-1], 1c3a [8-5-1] und 1c4a [1-2-1] von doppelkonischen Bechern, 1e4b [2-4-2] und 1e4c [1-1-1] von Näpfen, 2a1e [10-8-1], 2a2a [4-4-1] und 2a2e [10-7-1] von Butmir-Gefäßen.

${ }^{48}$ Typengruppen $1 \mathrm{~b} 2 \mathrm{e}$ [4-7] und $1 \mathrm{~b} 5 \mathrm{~g}$ [1-6] von dreigliedrigen Bechern, 1e4f [1-3] von Näpfen.

${ }^{49}$ Typengruppen 1b1b [1-4-2], 1b1e [4-3-7], 1b2a [18-194], 1b2b [6-3-6], 1b2d [1-4-4] und 1b5d [2-2-2] von dreigliedrigen Bechern, 1e2f [7-10-4] und 1e3b [2-15-1] von Näpfen und 2b3g [1-1-1] von Schüsseln. 
p) Das Vorkommen der Typengruppe 1b5h der dreigliedrigen Becher [1-2] ist nur in Svodin und Friebritz belegt.

q) Die Keramiken der Typengruppe 1b5m der dreigliedrigen Becher [1-1-1] kam nur in Alsónyék, Svodín und Friebritz zum Vorschein.

\subsection{Vorkommen und Verteilung der Formvarianten}

Die größten Übereinstimmungen und Ähnlichkeiten zwischen den untersuchten Keramikgefäßen treten im Fall der Typen in den Formen auf. Bei den Formvarianten kann man eine noch viel größere Streuung nach Regionen/ Fundorten erfahren als im Fall der vorigen typologischen Einheiten. Das Vorkommen und die Verteilung der 384 Varianten zeigen folgendes Bild.

a) Ein beachtenswertes Phänomen ist es, dass 38,28 \% $(n=147)$ der definierten keramischen Varianten aus dem Verbreitungsgebiet der Lengyel-Kultur im westlichen Karpatenbecken allein die Gräberfelder der südtransdanubischen Altgrabungen charakterisiert. ${ }^{50}$

b) 8,07\% $(\mathrm{n}=31)$ der Varianten tritt nur in den Gräbern von Alsónyék auf. ${ }^{51}$

c) Die Proportion der ausschließlich in Svodín nachgewiesenen Varianten beträgt $12,50 \%(\mathrm{n}=48) .{ }^{52}$

d) Nicht mehr als 5,21\% $(\mathrm{n}=20)$ der Varianten ist lediglich in Lužianky bekannt. ${ }^{53}$

e) Die nur in Veszprém vorhandenen Varianten treten mit einem Prozentsatz von nicht mehr als $1,30 \%(n=5)$ auf (Varianten 1a2h1 und 1a3d1 von Fußgefäßen, 1c2a1 von doppelkonischen Bechern, 1e4b3 und 1e4c3 von Näpfen).

f) Es gibt keine Variante, die allein für die Gräbergruppe von Friebritz kennzeichnend wäre.

g) Der Anteil der nur im Material der Altgrabungen und von Alsónyék befindlichen Varianten nimmt sogar $16,15 \%(\mathrm{n}=62)$ ein. ${ }^{54}$

h) Nicht mehr als $1,04 \%(n=4)$ der Varianten stammt allein aus den Altgrabungen und aus Lužianky. ${ }^{55}$

i) Auch aus chronologischer Sicht ist es wichtig, dass die Proportion der nur im Bestand der Altgrabungen sowie in Svodín belegten Varianten $10,94 \%$ (42) beträgt. ${ }^{56}$

${ }^{50}$ Varianten 1a1a2, 1a1b1, 1a1b2, 1a2c2, 1a2f1, 1a2f2, $1 \mathrm{a} 2 \mathrm{~g} 1,1 \mathrm{a} 2 \mathrm{j} 1,1 \mathrm{a} 2 \mathrm{j} 4,1 \mathrm{a} 2 \mathrm{k} 2,1 \mathrm{a} 2 \mathrm{~L} 1,1 \mathrm{a} 2 \mathrm{~L} 3,1 \mathrm{a} 3 \mathrm{a} 2,1 \mathrm{a} 3 \mathrm{~b} 2,1 \mathrm{a} 3 \mathrm{c} 1$, 1a3c2, 1a3e1, 1a3e3, 1a3e4, 1a3f1, 1a3f2, 1a3g1, 1a3h1, 1a3j2, $1 \mathrm{a} 3 \mathrm{k} 2,1 \mathrm{a} 3 \mathrm{k} 3,1 \mathrm{a} 4 \mathrm{~b} 1,1 \mathrm{a} 4 \mathrm{~b} 2,1 \mathrm{a} 4 \mathrm{c} 1,1 \mathrm{a} 4 \mathrm{c} 2,1 \mathrm{a} 4 \mathrm{c} 3,1 \mathrm{a} 4 \mathrm{c} 4,1 \mathrm{a} 4 \mathrm{~d} 1$ und 1a4d2 von Fußgefäßen, 1b1c2, 1b1e5, 1b1f2, 1b1f3, 1b2a8, 1b2c1, 1b2e1, 1b2f1, 1b2f2, 1b2f3, 1b2f5, 1b3a5, 1b3c1, 1b3c4, 1b3c5, 1b3d1, 1b3e2, 1b3e4, 1b4b1, 1b4c2, 1b4d1, 1b4e1, 1b4e2, 1b4e4, 1b4e5, 1b4e6, 1b4f3, 1b4i1, 1b5b1, 1b5b2, 1b5c2, 1b5e1 und $1 \mathrm{~b} 512$ von dreigliedrigen Bechern/Schultergefäßen, 1c1a2, 1c2b1, 1c2b6, $1 \mathrm{c} 2 \mathrm{~b} 7,1 \mathrm{c} 2 \mathrm{~b} 8,1 \mathrm{c} 3 \mathrm{a} 2,1 \mathrm{c} 3 \mathrm{a} 3$ und 1c4a 2 von doppelkonischen Bechern, $1 \mathrm{~d} 1 \mathrm{a} 1,1 \mathrm{~d} 1 \mathrm{a} 2,1 \mathrm{~d} 1 \mathrm{~b} 1,1 \mathrm{~d} 1 \mathrm{~b} 2,1 \mathrm{~d} 1 \mathrm{~b} 3,1 \mathrm{~d} 1 \mathrm{~b} 4,1 \mathrm{~d} 2 \mathrm{a} 2,1 \mathrm{~d} 2 \mathrm{a} 3$ und $1 \mathrm{~d} 2 \mathrm{a} 4$ von kugeligen Bechern, 1e1a4, 1e2f5, 1e2f6, 1e2f7, 1e2f8, 1e2g1, $1 \mathrm{e} 3 \mathrm{a} 1,1 \mathrm{e} 3 \mathrm{a} 4,1 \mathrm{e} 3 \mathrm{c} 3,1 \mathrm{e} 3 \mathrm{c} 4,1 \mathrm{e} 4 \mathrm{c} 2,1 \mathrm{e} 4 \mathrm{~d} 1$ und $1 \mathrm{e} 4 \mathrm{e} 1$ von Näpfen, 2a1a1, 2a1b3, 2a1c1, 2a1c2, 2a1d1, 2a1e3, 2a2d3, 2a2f1, 2a3b2 und $2 \mathrm{a} 3 \mathrm{c} 2$ von Butmir-Gefäßen, 2b1a1, 2b1b1, 2b2b1, 2b2b2, 2b2c1, 2b2d2, 2b3a5, 2b3b1, 2b3b3, 2b3d1, 2b3e1, 2b3e2, 2b3h1, 2b3i1, 2b4a1, 2b4a2, 2b4a3, 2b4a5, 2b4a9, 2b4c1, 2b5a1, 2b5a2, 2b5c1 und $2 \mathrm{~b} 5 \mathrm{~d} 1$ von Schüsseln, 2c1b1, 2c1b2, 2c1c1, 2c1c2, 2c1c3, 2c1c4, $2 \mathrm{c} 1 \mathrm{~d} 1,2 \mathrm{c} 1 \mathrm{~d} 2,2 \mathrm{c} 2 \mathrm{a} 1,2 \mathrm{c} 2 \mathrm{c} 1,2 \mathrm{c} 2 \mathrm{c} 2,2 \mathrm{c} 2 \mathrm{~d} 3,2 \mathrm{c} 2 \mathrm{~d} 5,2 \mathrm{c} 2 \mathrm{f} 1,2 \mathrm{c} 2 \mathrm{f} 2$ und $2 \mathrm{c} 2 \mathrm{f} 3$ von Schalen.

${ }^{51}$ Varianten 1a2k1, 1a2L2, 1a3i1 und 1a4a1 von Fußgefäßen, 1b1f1, 1b2f4, 1b3c2, 1b3c3, 1b3e1, 1b4i5, 1b5i1, 1b5i2, 1b5j1, $1 \mathrm{~b} 5 \mathrm{j} 2$ und $1 \mathrm{~b} 511$ von dreigliedrigen Bechern, 1d1b5, 1d2a1 und 1d2a5 von kugeligen Bechern, 1e1b1, 1e2c1, 1e2c2, 1e2c3, 1e3a2, 1e3c5 und $1 \mathrm{e} 4 \mathrm{c} 1$ von Näpfen, 2a1b1 und 2a2f2 von Butmir-Gefäßen, 2b3a2, $2 \mathrm{~b} 3 \mathrm{~d} 2$ und $2 \mathrm{~b} 5 \mathrm{~b} 1$ von Schüsseln, $2 \mathrm{c} 2 \mathrm{e} 1$ von Schalen.

${ }^{52}$ Varianten 1a1a1, 1a2a1, 1a2d2, 1a2i1, 1a3a1, 1a3d3, $1 \mathrm{a} 3 \mathrm{~d} 4$ und 1a3j1 von Fußgefäßen, 1b1b3, 1b1d1, 1b1d2, 1b2a4, 1b2a6, 1b2d2, 1b3a2, 1b3a3, 1b3a4, 1b3a6, 1b4a1, 1b4a4, 1b4e3, 1b5a1, 1b5a2, 1b5f1 und 1b5m2 von dreigliedrigen Bechern/Schultergefäßen, 1e1a1, 1e1a2, 1e2b2, 1e2f1, 1e3b1, 1e3b2, 1e3b3, 1e3c1, $1 \mathrm{e} 3 \mathrm{c} 2,1 \mathrm{e} 3 \mathrm{c} 6$ und $1 \mathrm{e} 4 \mathrm{a} 1$ von Näpfen, $2 \mathrm{a} 3 \mathrm{~b} 1,2 \mathrm{a} 3 \mathrm{c} 1$ und $2 \mathrm{a} 3 \mathrm{c} 3$ von Butmir-Gefäßen, 2b2d1, 2b2d3, 2b3a1, 2b3c1, 2b4b1, 2b4b2, 2b5e1 und $2 \mathrm{~b} 5 \mathrm{f} 1$ von Schüsseln, 2c1a1 von Schalen.

${ }^{53}$ Varianten 1a2b1 von Fußgefäßen, 1b1a1, 1b1a2, 1b1b2, 1b1e2, 1b1e3, 1b1e6, 1b1e7, 1b2b1, 1b2d3, 1b2d4, 1b2e2, 1b5d1 und $1 \mathrm{~b} 5 \mathrm{~g} 2$ von dreigliedrigen Bechern/Schultergefäßen, 1e2a1 und 1e4f1 von Näpfen, 2a2g1 von Butmir-Gefäßen, 2b3f1, 2b3f2 und 2b3g1 von Schüsseln.

${ }^{54}$ Varianten 1a1a3 [3-6], 1a1a4 [3-1], 1a2e1[1-1], 1a2j2 [1-2], 1a2k3 [1-1], 1a2k4 [3-1], 1a2k5 [1-1],1a214 [2-1], 1a3e2 [5-1], 1a3k1 [1-1], 1a3k4 [3-2] und 1a4c5 [1-1] von Fußgefäßen, 1b3e3 [21], 1b3e5 [2-3], 1b4f1 [8-1], 1b4f2 [1-3], 1b4g1 [1-1], 1b4h1 [1-2], 1b4i2 [3-5], 1b4i4 [3-2], 1b4i6 [2-1] und 1b5k1 [1-2] von dreigliedrigen Bechern/Schultergefäßen, 1c1a1 [1-1], 1c2b2 [2-3], 1c2b4 [1-2], $1 \mathrm{c} 2 \mathrm{~b} 5$ [1-1], 1c3a4 [4-1] und 1c3a5 [2-3] von doppelkonischen Bechern, 1e1a3 [1-1], 1e2c4 [1-2], 1e2d1 [3-1] und 1e4b1 [1-1] von Näpfen, 2a1b2 [2-1], 2a1e2 [4-2], 2a1e4 [2-3], 2a1e5 [1-3], 2a2a2 [2-2], 2a $2 \mathrm{a} 3$ [1-1], 2a2d1 [1-3], 2a2d2 [1-2], 2a2d4 [1-4], 2a2e1 [4-5] und 2a2e2 [3-2] von Butmir-Gefäßen, 2b1b2 [1-2], 2b1b3 [1-1], $2 \mathrm{~b} 2 \mathrm{a} 1$ [2-1], 2b2c2 [3-2], 2b2c3 [3-2], 2b2e1 [3-3], 2b2e 2 [1-1], 2b3a3 [2-4], 2b3a4 [4-1], 2b3b2 [2-3], 2b3b4 [1-1], 2b4a4 [2-1], 2b4a6 [1-2], 2b4a7 [2-1] und 2b4a8 [7-1] von Schüsseln, 2c1d3 [1-1], $2 \mathrm{c} 2 \mathrm{~d} 1$ [2-1], 2c2d2 [3-1] und 2c2d4 [4-1] von Schalen.

${ }^{55}$ Varianten 1b2a9 [1-2], 1b5d2 [1-1] und 1b5g1 [1-2] von dreigliedrigen Bechern/Schultergefäßen, 1e4f2 [1-1] von Näpfen.

${ }^{56}$ Varianten 1a2d1 [1-1], 1a2i2 [1-1], 1a3b1 [4-2] und $1 \mathrm{a} 3 \mathrm{~d} 2$ [1-3] von Fußgefäßen, 1b1c1 [9-2], 1b1e4 [2-1], 1b2a1 [4-8], 


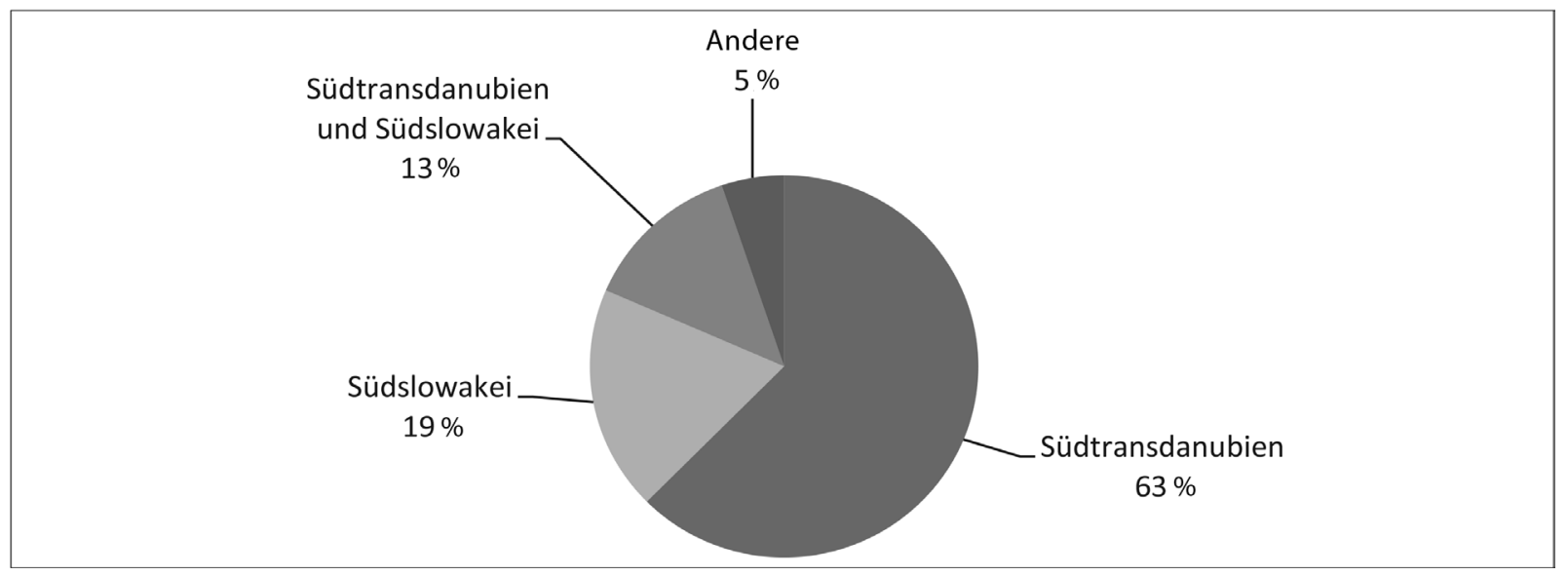

Diagramm 6. Vorkommen und Verteilung der keramischen Formvarianten nach Hauptregionen der Lengyel-Kultur

j) Die Varianten 1b3a1 [1-2-2] und 1b4a2 [1-2-1] der dreigliedrigen Becher sind als die alleinigen zu betrachten, die sowohl im Material der Altgrabungen als auch in Svodín und Friebritz erscheinen $(0,52 \%, \mathrm{n}=2)$.

k) Der Anteil der nur aus den Altgrabungen, in Svodín und Lužianky bestätigten Varianten macht nicht mehr als $1,04 \%(n=4)$ aus. ${ }^{57}$

1) Die Napfvariante 1e3a3 [1-1] erscheint allein in Alsónyék und Svodín. festgestellt. ${ }^{58}$

m) $0,52 \%(n=2)$ der Varianten wurde nur unter den Funden der Altgrabungen, von Alsónyék und Veszprém

n) $1,30 \%(\mathrm{n}=5)$ der Varianten dürfte auf relativchronologische Beziehungen zwischen den Nekropolen der Altgrabungen und der Gräbergruppe von Veszprém hinweisen. ${ }^{59}$

o) Mit einer ähnlichen Proportion sind Varianten vorhanden, die allein in Svodín und Lužianky bestätigt werden können. ${ }^{60}$

p) Nur in Svodin und Fribritz belegte Varianten sind durch $0,78 \%(\mathrm{n}=3)$ vertreten. $^{61}$

q) Ebenso 0,78 \% $(n=3)$ der Varianten dürfte auf engere relativchronologische Beziehungen zwischen Alsónyék und Veszprém hindeuten. ${ }^{62}$

Die nur in Südtransdanubien (Altgrabungen und Alsónyék) auftretenden Varianten sind durch mehr als die Hälfte, durch 62,50\% ( $\mathrm{n}=240)$, aller untersuchten keramischen Formvarianten (Typen) vertreten. Der Anteil der allein in der Südslowakei (Svodín mit Lužianky und Mlynárce/Molnos) nachgewiesenen Varianten beträgt dagegen nicht mehr als 19,01\% ( $\mathrm{n}=73)$. Ein beachtenswertes Phänomen ist es gleichzeitig, dass die Varianten, die sowohl in Südtransdanubien als auch in der Südslowakei belegt sind, mit einem Prozentsatz von 13,28 \% $(n=51)$ in den Vordergrund treten. Varianten von anderen Regionen bzw. Zusammenhänge des Lengyel-Kreises (Veszprém, Alsónyék mit Veszprém, Svodín mit Friebritz, Südtransdanubien mit Veszprém bzw. Altgrabungen mit Svodín und Friebritz) machen nicht mehr als 5,21\% $(\mathrm{n}=20)$ aus (Diagramm 5, Diagramm 6).

1b2a2 [7-1], 1b2a3 [3-2], 1b2a7 [2-3], 1b2b3 [6-1], 1b2d1 [1-1], $1 \mathrm{~b} 3 \mathrm{~b} 1$ [2-4], 1b3b2 [4-10], 1b3b3 [2-5], 1b3b4 [1-1], 1b3b5 [1-5], $1 \mathrm{~b} 3 \mathrm{~b} 6$ [1-1], 1b4a3 [2-7], 1b4c1 [6-4], 1b5b3 [4-2], 1b5c3 [1-2], $1 \mathrm{~b} 5 \mathrm{c} 4[1-1]$ und $1 \mathrm{~b} 5 \mathrm{~d} 3$ [1-2] von dreigliedrigen Bechern/Schultergefäßen, 1e2b1 [1-3], 1e2e1 [1-1], 1e2f2 [2-4] und 1e2h1 [1-2] von Näpfen, 2a2b1 [1-1], 2a2c1 [4-1], 2a3a1 [2-3], 2a3c4 [3-3] und 2a3c5 [1-1] von Butmir-Gefäßen, 2b2e3 [1-2], 2b3g2 [1-1], 2b3h2 [2-1], 2b3h3 [1-5], 2b3h4 [1-1], 2b3i2 [2-4] und 2b4c2 [2-1] von Schüsseln, $2 \mathrm{c} 1 \mathrm{a} 2$ [2-1] und 2c2b1 [1-2] von Schalen.

${ }^{57}$ Varianten 1e3b4 [2-1-1] von Näpfen, 1b1b1 [1-3-1], 1b1e1 [1-2-1] von dreigliedrigen Bechern/Schultergefäßen und 1e2f4 [1-3-1] von Näpfen.

\footnotetext{
${ }^{58}$ Varianten 1e4b2 [1-3-1] von Näpfen, 2a2a1 [1-1-1] von Butmir-Gefäßen.

${ }^{59}$ Varianten 1a2j3 [2-1] von Fußgefäßen, 1b4i3 [1-3] von dreigliedrigen Bechern/Schultergefäßen, 1c2b3 [1-1] von doppelkonischen Bechern, 2a1e1 [2-1] und 2a2e3 [3-1] von Butmir-Gefäßen.

${ }^{60}$ Varianten 1b2a5 [1-1], 1b2a10 [2-1] und 1b2b2 [2-4] von dreigliedrigen Bechern/Schultergefäßen, 1e2f3 [1-3] von Näpfen, 2b2f1 [5-1] von Schüsseln.

${ }^{61}$ Varianten 1a2c1 [1-1] von Fußgefäßen, 1b5c1 [1-1] und 1b5h1 [1-2] von dreigliedrigen Bechern/Schultergefäßen.

${ }^{62}$ Varianten $1 \mathrm{~b} 5 \mathrm{~m} 1$ [1-1] von dreigliedrigen Bechern/Schultergefäßen, 1c3a1 [1-1] und 1c4a1 [2-1] von doppelkonischen Bechern.
} 


\section{ERGEBNISSE DER SERIATION DER FUNDE UND BEFUNDE}

Nachdem die typologischen Einheiten der lengyelzeitlichen Grabkeramik aus dem westlichen Karpatenbecken nach Serien, Typengruppen und Formvarianten - also immer näher zur Ähnlichkeit - bestimmt wurden, versuchten wir eine relativchronologische Entwicklung der untersuchten Gräberfelder festzustellen. Mit Hilfe der Seriation studierten wir zuerst die Entwicklung der Typengruppen und zweitens die der Formvarianten der Grabkeramik. ${ }^{63}$

\subsection{Verteilung der keramischen Typengruppen}

Die Seriation nach Typengruppen wurde auf Grund von 145 Typengruppen vorgenommen. Die zeitliche Reihenfolge konnten wir dadurch bei 116 Typengruppen und 260 Gräbern feststellen. Die Ergebnisse der Hauptkoordinatenanalyse der Typengruppen stellt Tabelle 1, anhand von fünf Seriationsgruppen (Sg.), dar.

Auf die Analyse dieser Daten gestützt setzten wir die folgenden Seriationsgruppen ein.

1. $19,83 \%(n=30)$ der Typengruppen kann der Seriationsgruppe 1 zugeordnet werden. ${ }^{64}$ Die Daten der Hauptkoordinatenanalyse weisen darauf hin, dass 7 Typengruppen dieser Seriationsgruppe $(6,03 \%)$ in eine frühere Zeitperiode datiert werden dürfen wie die anderen. Als ein Ergebnis der Seriation wird ersichtlich, dass mehr als die Hälfte, 61,76 \% ( $\mathrm{n}=20)$, des Vorkommens dieser Typengruppen aus Lužianky stammt. Svodín wird mit einem Prozentsatz von 14,71 \% $(n=5)$ und Zengővárkony durch 17,65 \% $(n=6)$ vertreten. Györe tritt mit einem Anteil von $5,88 \%(\mathrm{n}=2)$ der Vorkommen in Südtransdanubien auf.

Die Situation ändert sich in der Seriationsgruppe 1b der Typengruppen insofern, dass Lužianky nur noch durch einen Anteil von 12,88 \% $(\mathrm{n}=21)$ vertreten wird, aber mehr als die Hälfte der Vorkommen schon für Svodín kennzeichnend ist. 21,47\% $(\mathrm{n}=35)$ der Vorkommen dieser Typengruppen erscheint in Zengővárkony, 4,91\% $(\mathrm{n}=8)$ in Györe, 1,20\% $(\mathrm{n}=2)$ in Pári-Altacker und 2,40 \% $(\mathrm{n}=4)$ in Friebritz. (Ein solches Vorkommen ist auch in Mórágy bekannt.) Diese frühesten Typengruppen sind im Gräberfeld von Alsónyék noch nicht nachgewiesen (Tabelle 1).

2. Die zweite Seriationsgruppe ist durch $17,24 \%(n=20)$ vertreten. ${ }^{65}$ Für Lužianky kennzeichnende Typengruppen treten nur noch mit einem Anteil von 1,90 \% $(n=4)$ auf. Mehr als die Hälfte, 50,98 \% (n=104), der Typengruppen stammt allein aus Svodín und 30,39\% $(\mathrm{n}=62)$ aus Zengővárkony. Das Gräberfeld von Mórágy zeichnet sich mit 6,86\% $(\mathrm{n}=14)$ aus. In Pári-Altacker sind diese Typengruppen durch 0,90 \% $(\mathrm{n}=2)$ und in Szekszárd-Ágostonpuszta durch 0,49\% $(\mathrm{n}=1)$ vertreten. Beachtenswert ist, dass solche keramischen Einheiten sowohl in Alsónyék als auch in Veszprém-Jutasi út mit 0,90\% $(n=2)$ und $0,49 \%(n=1)$ erscheinen.

3. 9,48\% $(n=11)$ gehört zur Seriationsgruppe 3 nach Typengruppen. ${ }^{66}$ In dieser Seriationsgruppe zeichnet sich Zengővárkony mit mehr als die Hälfte, mit 58,73 \% $(n=37)$, und Mórágy mit einem Prozentsatz von 20,63\% $(\mathrm{n}=13)$ der Vorkommen der Typengruppen aus. Das Gräberfeld von Svodín ist nur mit 9,52 \% $(\mathrm{n}=6)$ vertreten. In Villánykövesd, Pécsvárad, Pári-Altacker und Szekszárd-Ágostonpuszta erscheinen sie mit je 1,59 \% $(\mathrm{n}=1-1)$ und in Pécsvárad mit 3,17 \% $(\mathrm{n}=2)$. Für das Gräberfeld von Alsónyék ist 3,17 \% $(\mathrm{n}=2)$ der Vorkommen dieser Typengruppen typisch.

63 „Seriationen können eine relativchronologische Abfolge von unterschiedlichen Typenvertretern oder Befunden ermitteln. Unter einer Seriation versteht man die Anordnung von Typenvertretern in einer linearen Serie. Diese ist so organisiert, dass die Position jedes Typenvertreters innerhalb einer Matrix optimal den Grad der Ähnlichkeit zwischen ihm und den übrigen Typenvertretern in dieser Anordnung wiedergibt“ (http://www.praehistorische-archaeologie.de/ wissen/datierung/korrespondenzanalyse).

${ }^{64}$ Es handelt sich dabei um die Typengruppen 1a2a, 1a2c, 1a3a, 1a3b, 1a3j, 1a2i und 1a2d von Fußgefäßen, 1b1a, 1b1b, 1b1d, 1b2d, 1b1e, 1b2b, 1b2e, 1b3a, 1b5a, 1b5c, 1b5d, 1b5g und 1b5h von
Bechern/Schultergefäßen, 1e2f, 1e 3b und 1e4f von Näpfen, 2a2b von Butmir-Gefäßen, 2b2f, 2b3f, 2b3h, 2b4b und 2b4c von Schüsseln und 2c1a von Schalen.

${ }^{65}$ Hierbei sollen wir Typengruppen $1 \mathrm{a} 3 \mathrm{c}, 1 \mathrm{a} 3 \mathrm{~d}$ und $1 \mathrm{a} 4 \mathrm{~b}$ von Fußgefäßen, 1b1c, 1b2a, 1b3b, 1b4a, 1b4c und 1b5b von Bechern/Schultergefäßen, 1e1a, 1e2b und 1e3c von Näpfen, 2a1a, 2a3a, $2 \mathrm{a} 3 \mathrm{~b}$ und $2 \mathrm{a} 3 \mathrm{c}$ von Butmir-Gefäßen, 2b2b, 2b2d, 2b3g und 2b3i von Schüsseln sowie $2 c 2 b$ von Schalen erwähnen.

${ }^{66}$ Typengruppen 1a1b, 1a3e, 1a3h und 1a4d von Fußgefäßen, 1b4d, 1b4e und 1b51 von Bechern/Schultergefäßen, 1e2e von Näpfen, 2a2c von Butmir-Gefäßen und 2c1b bzw. 2c1c von Schalen. 
Tabelle 1. Vorkommen der Seriationsgruppen in den untersuchten Nekropolen

\begin{tabular}{|c|c|c|c|c|c|}
\hline \multirow{2}{*}{ Gräberfelder } & \multicolumn{5}{|c|}{ Seriationsgruppen nach Typengruppen } \\
\hline & Sg. 1 & Sg. 2 & Sg. 3 & Sg. 4 & Sg. 5 \\
\hline Friebritz & 4 & & & & \\
\hline Lužianky & 21 & 4 & & & \\
\hline Györe & 8 & 13 & & & \\
\hline Svodín & 92 & 104 & 6 & 8 & 1 \\
\hline Zengővárkony & 35 & 62 & 37 & 74 & 61 \\
\hline Mórágy & 1 & 14 & 13 & 44 & 95 \\
\hline Pári & 2 & & 1 & 3 & 2 \\
\hline Ágostonpuszta & & 1 & 1 & & \\
\hline Veszprém & & 1 & & 8 & 6 \\
\hline Alsónyék & & 2 & 2 & 68 & 99 \\
\hline Villánykövesd & & & 1 & 4 & 13 \\
\hline Pécsvárad & & & 2 & 1 & 4 \\
\hline
\end{tabular}

4. Die Typengruppen nach Seriationsgruppe 4 sind ebenfalls mit einem Prozentsatz von $17,24 \%(n=20)$ vorhanden. ${ }^{67}$ Hierbei zeichnet sich das Gräberfeld von Zengővárkony im größten Verhältnis, um 35,24 \% $(\mathrm{n}=74)$ der Vorkommen dieser Typengruppen, aus, darauf folgt Alsónyék mit einem Prozentsatz von 32,38 \% $(\mathrm{n}=68)$ und Mórágy mit 20,95\% $(\mathrm{n}=44)$ mit neuen Typengruppen. Aus Sicht der relativchronologischen Stellung der hier diskutierten Lengyel-Nekropolen ist es wichtig, dass auch die Gräbergruppe von Veszprém mit 3,81 \% $(n=8)$ der Vorkommen jetzt schon repäsentiert wird und auch Svodín Typengruppen der Seriationsgruppe 4 in demselben Verhältnis aufweist. Es sind noch hier die Gräberfelder von Villánykövesd (1,90\%, n=4), Pári-Altacker $(1,43 \%$, $\mathrm{n}=3)$ und Pécsvárad $(0,40 \%, \mathrm{n}=1)$ zu erwähnen.

5. Mit dem größten Prozentsatz, mit 30,17\% $(n=35)$, sind Typengruppen nach Seriationsgruppe 5 nachgewiesen. ${ }^{68}$ Das Gräberfeld von Alsónyék ragt diesmal mit 35,23\% $(\mathrm{n}=99)$, Mórágy mit 33,81 \% $(\mathrm{n}=95)$ und Zengővárkony mit 27,71 \% $(\mathrm{n}=61)$ heraus. Villánykövesd vertritt dann einen Anteil von 4,63\% $(\mathrm{n}=13)$, Pécsvárad $1,42 \%(\mathrm{n}=4)$ und Pári-Altacker 0,71\% $(\mathrm{n}=2)$. Auch diesmal sind die in der vorherigen Seriationsgruppe erfahrenen Phänomene erkennbar: In Svodín ist jetzt nur eine alleinige Typengruppe nachweisbar, die Gräbergruppe von Veszprém kann dagegen schon mit 2,10\% $(n=6)$ der Vorkommen dieser Typengruppen gekennzeichnet werden (Tabelle 1).

\subsection{Verteilung der Gräber nach Seriation der Typengruppen}

Die im Vorherigen skizzierten Seriationsgruppen der keramischen Typengruppen dürften die relativchronologische Entwicklung der lengyelzeitlichen Grabkeramik bzw. der Gräber und Gräberfelder im westlichen Karpatenbecken gespiegelt haben. Im nächsten Abschnitt der vorliegenden Arbeit wird nach der relativchronologischen Stellung der untersuchten Bestattungen und Gräberfelder gesucht. Die Verteilung der seriierten Gräber nach fünf künstlichen Perioden (kP) wird auf $A b b .43$ vorgezeigt.

1. Der künstlichen Periode 1 (kP1) nach Typengruppen der Grabkeramik konnte 18,08\% $(\mathrm{n}=47)$ der nach Vorkommen von Typengruppen seriierten Gräber aus fünf Nekropolen zugeordnet werden. Diese Einheit der Bestattungen kann man anhand der Seriation in zwei Gruppen einteilen.

${ }^{67}$ Typengruppen 1a1a, 1a2j, 1a2l, 1a3c, 1a3f und 1a3k von Fußgefäßen, 1b2f, 1b3d, 1b4f, 1b4i, 1b5m, 1c3a und 1c4a von Bechern/Schultergefäßen, 1e4c von Näpfen, 2b2c, 2b2e, 2b3a, 2b3d und $2 \mathrm{~b} 4 \mathrm{a}$ von Schüsseln und $2 \mathrm{c} 2 \mathrm{f}$ von Schalen.

${ }^{68}$ Typengruppen 1a2f, 1a2k, 1a3g, 1a4c von Fußgefäßen, 1b1f, 1b2c, 1b3c, 1b3e, 1b4h, 1b5i, 1b5j, 1b5k, 1c1a, 1c2b, 1d1a, 1d1b, 1d2a, von Bechern/Schultergefäßen, 1e2c, 1e2d, 1e3a und 1e 4b von Näpfen, 2a1b, 2a1c, 2a1d, 2a1e, 2a2a, 2a2d, 2a2e und 2a $2 \mathrm{f}$ von Butmir-Gefäßen, 2b1b, 2b2a und 2b3b von Schüsseln bzw. 2c1d, 2c2c und $2 \mathrm{c} 2 \mathrm{~d}$ von Schalen. 


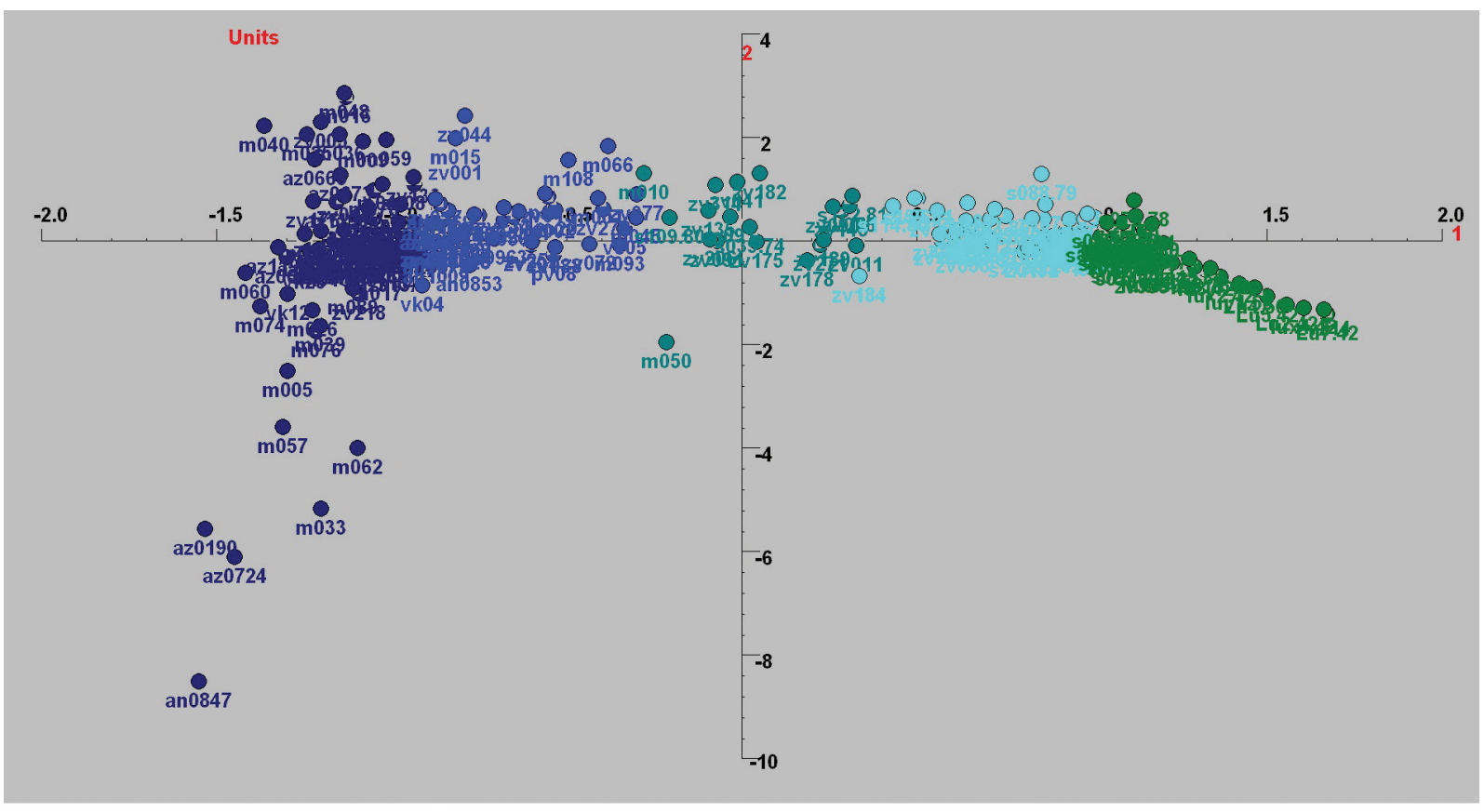

Abb. 43. Hauptkoordinatenanalyse der nach Typengruppen seriierten Gräber.

Abkürzungen: Lužianky ( Lu = Skelettgrab, lux = symbolische Bestattungen, luy = Kultgrube $), \mathbf{m o}=$ Mlynárce, $\mathbf{s v}=$ Svodín, $\mathbf{f r}=$ Friebritz, $\mathbf{v e}=$ Veszprém-Jutasi út, an = Fundstelle 10B von Alsónyék, az = Fundstelle 5603 von Alsónyék, ap = Szekszárd-Ágostonpuszta, gy = Györe, $\mathbf{m}=$ Mórágy, $\mathbf{p a}=$ Pári-Altacker, $\mathbf{p v}=$ Pécsvárad, $\mathbf{v k}=$ Villánykövesd, $\mathbf{z v}=$ Zengővárkony

Kp 1a: Dieser Gruppe nur mit 10 der seriierten Befunde können neun Gräber der Lužianky-Gruppe von dem namengebenden Fundort und Mlynárce ${ }^{69}$ sowie eventuell das Grab 214 aus Zengővárkony (Gräbergruppe 6c) zugeordnet werden.

Kp 1b: Von den hier gruppierten 37 Bestattungen stammen nur mehr zwei aus Lužianky, ${ }^{70}$ aber die Zahl der Gräber von Svodín beträgt diesmal 24. ${ }^{71}$ Auch die Gräber fr130 und fr131 von Friebritz bzw. gy9, gy 12 und gy13 aus Györe vertreten die behandelte künstliche Periode, wie die Bestattungen zv57 (Gräbergruppe 6a), zv173, zv177, zv179, zv186 (Gräbergruppe 6c) und zv355 (Gräbergruppe 13) von Zengővárkony.

2. Die Proportion der zur künstlichen Periode 2 nach Typengruppen gehörenden Lengyel-Bestattungen beträgt 20,38 \% $(n=53)$ in sechs Nekropolen. Ein solches Grab stammt aus Lužianky (1,89 \%, Grab lux5.42) und in Svodín befindet sich mehr als die Hälfte, 52,83\% $(\mathrm{n}=28)$, der Bestattungen der behandelten künstlichen Gruppe. ${ }^{72}$ Györe zeichnet sich mit zwei (Grab gy10 und gy11) solchen Befunden und Pári-Altacker mit einem Grab (pa2) aus. Auch drei Befunde aus Mórágy (5,66 \%, Grab m58, m77 und m109) konnten hier zugeordnet werden. Im Gräberfeld von Zengővárkony befinden sich sogar sieben Gräbergruppen mit einem Anteil von 33,96 \% $(\mathrm{n}=18)$ solcher Befunde. ${ }^{73}$

3. Die künstliche Periode 3 nach Typengruppen wird mit einem Prozentsatz von nicht mehr als 7,69\% $(n=20)$ und nur in drei Gräberfeldern repräsentiert. Je 15,0 \% $(n=3-3)$ dieser Bestattungen stellten wir in Svodín

${ }^{69}$ Grab lu2.42, lu3.42, lu5.42, lu4.56, lu7.42, lux2.42, lux 4.42, luy1.56, mo16.42.

${ }^{70}$ Grab lux 1.42 , lux3.42.

${ }^{71}$ Grab sv37.74, sv7.72, sv112.80, sv94.79, sv76.78, sv113.80, sv24.74, sv122.80, sv163.81, sv70.78, sv29.73, sv23.73, sv13.73, sv93.79, sv160.81, sv5.72, sv45.74, sv178.82, sv42.74, sv16.73, sv130.80, sv77.78, sv48.76 und sv143.81.

${ }^{72}$ Grab sv148.81, sv139.81, sv132.80, sv21.73, sv134.80, sv127.80, sv3.71, sv14.73, sv126.80, sv41.74, sv106.80, sv171.81, sv156.81, sv27.73, sv144.81, sv176.82, sv105.80, sv88.79, sv140.81, sv10.72, sv177.82, sv9.72, sv103.80, sv2.71, sv30.73, sv121.80, sv159.81 und sv114.80.

${ }^{73}$ Gräbergruppe 5 (Grab zv35), Gräbergruppe 6a (Grab zv56, zv59 und zv60), Gräbergruppe 6c (Grab zv174, zv180, zv181, zv184, zv187, zv188, zv205), Gräbergruppe 6d (Grab zv239), Gräbergruppe 7 (Grab zv79), Gräbergruppe 8b (Grab zv265), Gräbergruppe 9 (Grab zv93) und Gräbergruppe 13 (Grab zv311, zv360, zv361). 


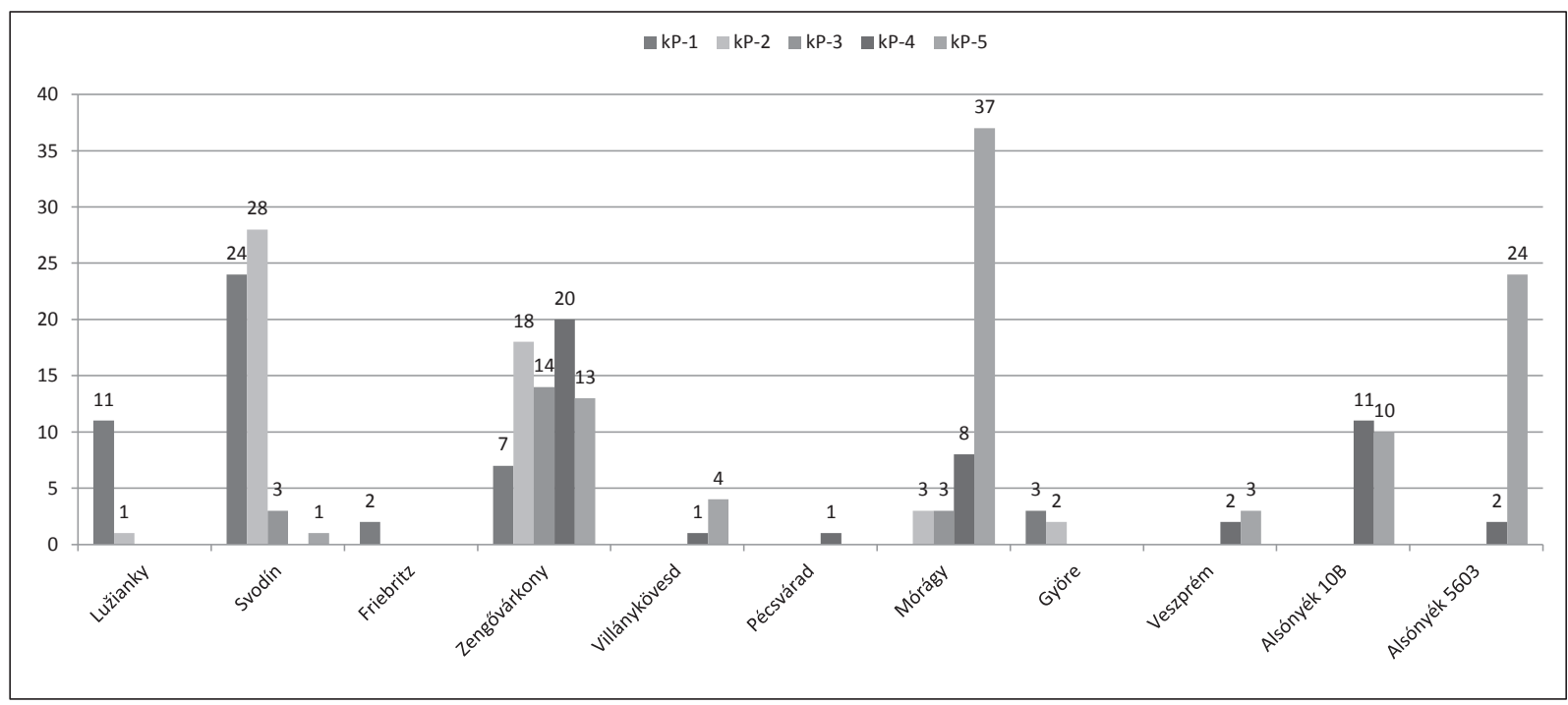

Diagramm 7. Verteilung der nach den Typengruppen seriierten Gräber und nach künstlichen Perioden in den größeren Lengyel-Nekropolen

und Mórágy fest. ${ }^{74} 70,0 \%(\mathrm{n}=14)$ von ihnen stammt gleichzeitig aus Zengővárkony und zwar aus sieben Gräbergruppen. ${ }^{75}$

4. 18,08\% $(\mathrm{n}=47)$ der nach Typengruppen untersuchten Bestattungen, schon aus neun Gräberfeldern, konnte der künstlichen Periode 4 zugeordnet werden. Zengővárkony zeichnet sich fast mit der Hälfte, mit 42,55 \% $(\mathrm{n}=20)$, der Bestattungen aus. ${ }^{76}$ Mórágy tritt diesmal mit 17,02 \% $(\mathrm{n}=8)$ der Bestattungen in den Vordergrund. ${ }^{77}$

Die ältesten Lengyel-Gräber von Alsónyék-Bátaszék erscheinen in dieser Zeit. Zwischen den zwei studierten Fundstellen von hier können dagegen beträchtliche Unterschiede beobachtet werden. 23,40 \% ( $\mathrm{n}=11)$ wurde nämlich an der Fundstelle 10B und nur 4,26 \% (n=2) an der Fundstelle 5603 festgestellt. ${ }^{78}$ Außerdem ist es beachtenswert, dass auch die ersten Bestattungen von Veszprém-Jutasi út (4,26\%, Grab ve5 und ve7) zu dieser Zeit erscheinen. In den Nekropolen von Villánykövesd (Grab vk4), Pécsvárad (Grab pv8), Szekszárd-Ágostonpuszta (Grab ap20) und Pári-Altacker (Grab pa5) ist je ein solches Grab vorhanden.

5. Im größten Verhältnis, mit 35,77 \% ( $\mathrm{n}=93)$, treten die Gräber der künstlichen Periode 5 nach Typengruppen auf. Mit dem größten Prozentsatz, mit 39,78 \% ( $=37)$, kommt schon das Gräberfeld von Mórágy ins Bild. ${ }^{79}$ Die Gräbergruppe (Fundstelle) 5603 von Alsónyék folgt ihm mit sogar 25,81\% $(\mathrm{n}=24) .{ }^{80}$ An der anderen Fundstelle von Alsónyék ist diese künstliche Periode mit nicht mehr als $10,75 \%(\mathrm{n}=10)$ der Gräber bestätigt. ${ }^{81}$ In Zengővárkony findet man nur 13,98 \% $(\mathrm{n}=13)$ dieser Bestattungen, und zwar in sechs Gräbergruppen. ${ }^{82}$ Ein beachtenswertes Phänomen ist es weiterhin, dass nur ein Befund (Grab sv6.72) in Svodín vorhanden ist, der die behandelte künstliche Periode vertritt. Auch drei andere Bestattungen (3,23\%, Grab ve2, ve3 und ve6) aus Veszprém

${ }^{74}$ Grab sv39.74, sv109.80, sv172.81 aus Svodín sowie Grab m10, m50 und m91 von Mórágy.

${ }^{75}$ Gräbergruppe 2b (Grab zv11), Gräbergruppe 5 (Grab zv41), Gräbergruppe 6b (Grab zv76), Gräbergruppe 6c (Grab zv175, zv178, zv182, zv189, zv206), Gräbergruppe 8b (Grab zv271), Gräbergruppe 9 (Grab zv90, zv91, zv135) und Gräbergruppe 14a (Grab zv314).

${ }^{76}$ Gräbergruppe 1 (Grab zv1), Gräbergruppe 2a (Grab zv17), Gräbergruppe 2b (Grab zv12, zv13), Gräbergruppe 6b (Grab zv77, zv72), Gräbergruppe 5 (Grab zv44, zv49), Gräbergruppe $6 a$ (Grab zv64), Gräbergruppe 6b (Grab zv72), Gräbergruppe 6c (Grab zv185, zv201), Gräbergruppe 6d (Grab zv234, zv247, zv252), Gräbergruppe 8b (Grab zv266), Gräbergruppe 9 (Grab zv88, zv326), Gräbergruppe 11 (Grab zv272) und Gräbergruppe 13 (Grab zv350).

${ }^{77} \mathrm{Grab}$ m1, m2, m15, m45, m56, m66, m93 und m108.
${ }^{78}$ Grab an769, an801, an804, an828, an853, an963, an1009, an1473, an3089, an4012, an4027 und Grab az337, az1669 von Alsónyék.

${ }^{79} \mathrm{Grab} \mathrm{m} 3, \mathrm{~m} 5, \mathrm{~m} 7, \mathrm{~m} 8, \mathrm{~m} 9, \mathrm{~m} 14, \mathrm{~m} 16, \mathrm{~m} 17, \mathrm{~m} 25, \mathrm{~m} 26$, $\mathrm{m} 27, \mathrm{~m} 33, \mathrm{~m} 34, \mathrm{~m} 36, \mathrm{~m} 37, \mathrm{~m} 38, \mathrm{~m} 39, \mathrm{~m} 40, \mathrm{~m} 41, \mathrm{~m} 43, \mathrm{~m} 44, \mathrm{~m} 46$, m48, m49, m51, m53, m55, m57, m59, m60, m62, m71, m76, m78, m80, m84 und m89.

${ }^{80}$ Grab az190, az228, az263, az272, az278, az304, az318, az319, az333, az665, az673, az699, az724, az743, az1006, az1190, az1191, az1235, az1320, az1391, az1967, az2028, az2330, az3132.

${ }^{81}$ Grab an396, an398, an792, an796, an811, an813, an847, an3020, an3103 und an4028.

${ }^{82}$ Gräbergruppe 2b (Grab zv5, zv14), Gräbergruppe 6c (Grab zv176, zv196, zv203, zv218), Gräbergruppe 6d (Grab zv232, zv238), Gräbergruppe 9 (Grab zv114, zv117, zv130), Gräbergruppe10 (Grab zv144), Gräbergruppe 12 (Grab 335). 
können in diese Periode eingereiht werden. Aus Villánykövesd stammt 4,30 \% ( $\mathrm{n}=4,30 \%)$ der Bestattungen und eine solche ist auch noch in Pári-Altacker belegt. ${ }^{83}$

Das Vorkommen und die Verteilung der nach Typengruppen seriierten Bestattungen in den größeren Lengyel-Nekropolen zeigt Diagramm 7.

\subsection{Vorkommen und Verteilung der keramischen Formvarianten (Typen)}

Die zeitliche Reihenfolge oder relativchronologische Entwicklung nach Varianten (Typen) der lengyelzeitlichen Grabkeramik konnte durch die Seriation von 219 Gräbern und 210 Typen bzw. insgesamt 758 Vorkommen festgestellt werden. ${ }^{84}$ Das Vorkommen von künstlichen Perioden nach Formvarianten in den einzelnen Nekropolen zeigt Tabelle 2.

Tabelle 2. Vorkommen der künstlichen Perioden nach Varianten in den untersuchten Nekropolen

\begin{tabular}{|c|c|c|c|c|c|}
\hline \multirow{2}{*}{ Gräberfelder } & \multicolumn{5}{|c|}{ Künstliche Perioden nach Formvarianten } \\
\hline & Kp. 1 & Kp. 2 & Kp. 3 & Kp. 4 & Kp. 5 \\
\hline Lužianky & 46 & & & & \\
\hline Friebritz & 3 & 1 & & & \\
\hline Györe & 11 & 9 & & & \\
\hline Svodín & 81 & 102 & 3 & & \\
\hline Zengővárkony & 11 & 76 & 43 & 54 & 12 \\
\hline Mórágy & 2 & 11 & 22 & 56 & 33 \\
\hline Alsónyék & & 2 & 23 & 109 & 11 \\
\hline Ágostonpuszta & & 2 & 1 & 1 & \\
\hline Pécsvárad & & 2 & & 1 & \\
\hline Villánykövesd & & & 2 & 11 & \\
\hline Veszprém & & & 2 & 1 & 6 \\
\hline
\end{tabular}

1. Die künstliche Periode 1 nach Varianten wird durch 20,32 \% $(n=154)$ aller Vorkommen und in nicht mehr als sechs Nekropolen vertreten. Das Gräberfeld von Lužianky zeichnet sich mit einem Prozentsatz von 29,87 \% ( $\mathrm{n}=46)$ und das Gräberfeld von Svodín mit mehr als die Hälfte, 52,60 \%, aus. Sowohl in Zengővárkony als auch in Györe erscheint diese Seriationsgruppe mit je 7,14 \%, in Friebritz mit 1,95 \% und in Mórágy mit 1,30 \% (Tabelle 2, Diagramm 8).

2. Die künstliche Periode 2 nach Varianten konnte schon in neun Gräberfeldern und mit einem Prozentsatz von 27,70 \% aller Vorkommen nachgewiesen werden. Svodín überwiegt auch in diesem Fall beinahe mit der Hälfte, mit 48,57 \%, der Vorkommen dieser Seriationsgruppe. Sie erscheint in Zengővárkony mit 36,19 \% und in Mórágy noch mit 5,24 \% der Fälle. Das Gräberfeld von Alsónyék kommt zuerst jetzt ins Bild, und zwar mit nur 0,95 \% der Vorkommen. Der Anteil der Seriationsgruppe nimmt in Györe dagegen 4,29 \% ein (Tabelle 2, Diagramm 8).

3. Die künstliche Periode 3 nach Varianten tritt mit einem Prozentsatz von 12,93\% ( $\mathrm{n}=98)$ auf. Die Situation ändert sich jetzt insofern, dass fast die Hälfte, d. h. 43,88 \%, der Vorkommen dieser Seriationsgruppe in Zengővárkony belegbar ist. Die Gräberfelder von Alsónyék und Mórágy sind ebenso mit hohen Prozentsätzen (mit 23,47 \% und 22,45 \%) repräsentiert. Im Fall von Svodín handelt es sich dagegen um die jüngsten Befunde des Gräberfeldes (3,06 \%) (Tabelle 2, Diagramm 8).

4. Die künstliche Periode 4 nach Varianten wird durch einen Anteil von 30,74 \% $(n=233)$ aller Vorkommen vertreten. In diesem Fall überwiegt Alsónyék schon beinahe mit der Hälfte, 46,78 \%, darauf folgen Mórágy mit 24,03 \% und Zengővárkony mit 23,18 \%. Die Gräbergruppe von Villánykövesd zeichnet sich mit 4,72 \% aus und je ein Vorkommen ist noch in Veszprém, Pécsvárad und Szekszárd-Ágostonpuszta zu finden (Tabelle 2, Diagramm 8).

\footnotetext{
${ }^{83}$ Grab vk10, vk12, vk20, vk23 von Villánykövesd und Grab pa6 von Pári-Altacker.

${ }^{84}$ Wegen der verringerten Datenmenge wurden mehrere Typen und Gräber durch das WINBASP-Programm exkludiert. 


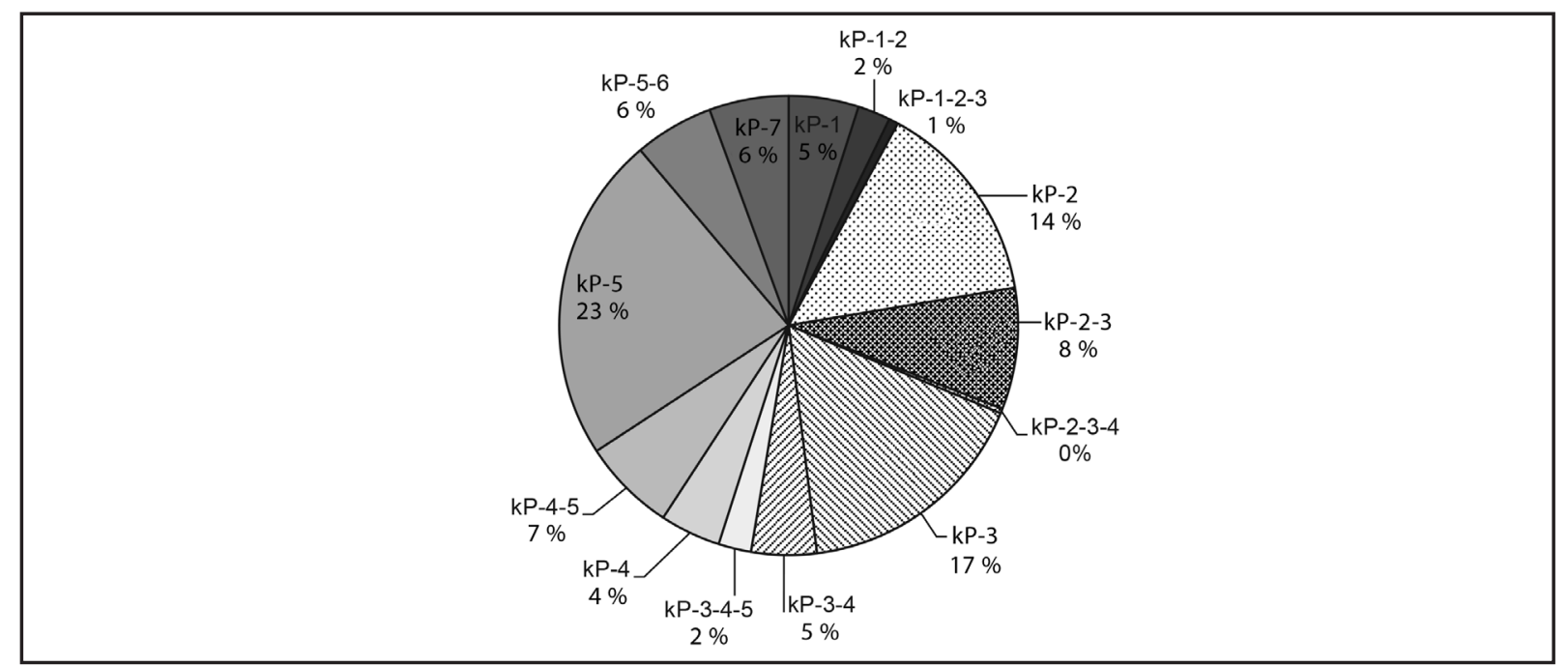

Diagramm 8. Verteilung der Vorkommen nach künstlichen Perioden in den Nekropolen der Lengyel-Kultur

5. Die Zahl der Vorkommen nimmt in der künstlichen Periode 5 nach Varianten stark ab $(8,31 \%, \mathrm{n}=63)$. Das Gräberfeld von Mórágy dominiert mit 52,38 \% der Fälle, für Zengővárkony und Alsónyék sind 19,05 \% und $17,46 \%$ der Vorkommen typisch. In Veszprém und Villánykövesd konnte je ein Vorkommen der erörterten Variante nachgewiesen werden (Tabelle 2).

Über das Vorkommen der keramischen Formvarianten nach künstlichen Perioden kann man Folgendes resümieren.

Es gibt fünfzehn keramische Varianten $(4,93 \%)$, die allein für die kP-1 typisch sind. ${ }^{85}$ Sieben andere Varianten $(2,30 \%)$ sind sowohl für die kP-1 als auch für die kP-2 belegt. ${ }^{86}$ Nicht mehr als zwei Varianten $(0,66 \%)$ sind vorhanden, die in den ersten drei künstlichen Perioden nachweisbar sind. ${ }^{87}$ Beachtenswert ist, dass die Zahl der nur in der kP-2 vorhandenen Formvarianten bedeutend gewachsen ist; es handelt sich um 44 keramische Typen $(14,47 \%) .{ }^{88} 26$ andere Formvarianten $(8,55 \%)$ befinden sich in der kP-2 sowie in der kP-3. ${ }^{89}$ Die Bechervariante 1b4c1 erscheint während der künstlichen Perioden 2, 3 und 4 (Tabelle 2, Diagramm 8).

Die künstliche Periode 3 zeichnet sich durch die enorm große Zahl von den neuen keramischen Formvarianten aus: Nicht weniger als 51 Varianten charakterisieren ausschließlich diese Periode. ${ }^{90}$ Die Zahl der während der kP-3 und kP-4 befindlichen Formvarianten ist $14(4,61 \%)^{91}$ und nicht mehr als 7 Varianten $(2,30 \%)$ treten sowohl in der kP-3 und kP-4 als auch in der kP-5 auf (Tabelle 2, Diagramm 8). ${ }^{92}$

${ }^{85}$ Varianten 1a2b1 von Fußgefäßen, 1b1a2, 1b1b2, 1b1e2, 1b1e6, 1b1e7, 1b2d3, 1b2d4, 1b2e2, 1b5g1 und 1b5g2 von Bechern, 1e4f1 von Näpfen, 2a2g1 von Butmir-Gefäßen, 2b3f1 und 2b3f2 von Schüsseln.

${ }^{86}$ Varianten 1b1e3, 1b2a5, 1b2a9, 1b2a10, 1b2b1 und 1b2b2 von Bechern/Schultergefäßen, 1e2f3 von Näpfen.

${ }^{87}$ Variante $1 \mathrm{~b} 1 \mathrm{e} 1$ von Bechern und 2b2f1 von Schüsseln.

${ }^{88}$ Varianten 1a2a1, 1a2c1, 1a2d2, 1a2i2, 1a3a1, 1a3a2, 1a3d2, 1a3d4 und 1a3j1 von Fußgefäßen, 1b1a1, 1b1b1, 1b1b3, 1b2a4, $1 \mathrm{~b} 2 \mathrm{a} 6,1 \mathrm{~b} 2 \mathrm{~d} 1,1 \mathrm{~b} 2 \mathrm{~d} 2,1 \mathrm{~b} 3 \mathrm{a} 3,1 \mathrm{~b} 3 \mathrm{a} 4,1 \mathrm{~b} 3 \mathrm{~b} 4,1 \mathrm{~b} 4 \mathrm{e} 3,1 \mathrm{~b} 5 \mathrm{c} 1,1 \mathrm{~b} 5 \mathrm{c} 2$, $1 \mathrm{~b} 5 \mathrm{c} 3,1 \mathrm{~b} 5 \mathrm{c} 4$ und $1 \mathrm{~b} 5 \mathrm{~h} 1$ von Bechern/Schultergefäßen, 1e1a1, 1e2f4, $1 \mathrm{e} 3 \mathrm{~b} 1,1 \mathrm{e} 3 \mathrm{~b} 2,1 \mathrm{e} 3 \mathrm{c} 1,1 \mathrm{e} 3 \mathrm{c} 4,1 \mathrm{e} 3 \mathrm{c} 6,1 \mathrm{e} 4 \mathrm{a} 1$ und 1e4f2 von Näpfen, $2 \mathrm{a} 2 \mathrm{~b} 1,2 \mathrm{a} 3 \mathrm{c} 3$ und $2 \mathrm{a} 3 \mathrm{c} 5$ von Butmir-Gefäßen, 2b2d1, 2b3g1, 2b2h3, $2 \mathrm{~b} 3 \mathrm{~h} 3,2 \mathrm{~b} 5 \mathrm{e} 1$ und $2 \mathrm{~b} 5 \mathrm{f} 1$ von Schüsseln bzw. 2c1a1 von Schalen.

${ }^{89}$ Varianten 1a2c2, 1a2i1 und 1a3b1 von Fußgefäßen, 1b1c1, 1b2a1, 1b2a3,1b2e1, 1b3a1, 1b3b1, 1b3b2, 1b3b3, 1b3b5, $1 \mathrm{~b} 4 \mathrm{a} 3$ und $1 \mathrm{~b} 5 \mathrm{a} 2$ von Bechern/Schultergefäßen, 1e2b1, 1e2f2, 1e3b3 und 1e3b4 von Näpfen, 2a3a1, 2a3c1 und 2a3c4 von Butmir-Gefäßen, 2b2d3, 2b3a1, 2b3h2, 2b3i2 und 2b4c2 von Schüsseln.

${ }^{90}$ Varianten 1a2d1, 1a2g1, 1a3c1, 1a3d3, 1a3e4, 1a3k2, 1a4b1, 1a4b2 und 1a4c4 von Fußgefäßen, 1b1e4, 1b2a8, 1b2b3, 1b2f5, 1b3a2, 1b3a6, 1b3b6, 1b4a2, 1b4e4, 1b5b1, 1b5b3, 1b5d2, $1 \mathrm{~b} 5 \mathrm{~d} 3,1 \mathrm{~b} 5 \mathrm{e} 1,1 \mathrm{~b} 5 \mathrm{f} 1,1 \mathrm{~b} 512$ und 1c2b6 von Bechern/Schultergefäßen, 1e1a3, 1e2h1, 1e2e1, 1e2f1, 1e2f8, 1e3a1 und 1e3c2 von Näpfen, 2a1a1 und 2a2c1 von Butmir-Gefäßen, 2b2b1, 2b2c1, 2b2d2, 2b3c1, 2b3h4, 2b3i1, 2b4b1 und 2b5c1 von Schüsseln, 2c1a2, 2c1b1, 2c1b2, $2 \mathrm{c} 1 \mathrm{c} 1,2 \mathrm{c} 1 \mathrm{c} 2,2 \mathrm{c} 1 \mathrm{c} 4,2 \mathrm{c} 1 \mathrm{~b} 1$ und $2 \mathrm{c} 2 \mathrm{~d} 3$ von Schalen.

${ }^{91}$ Varianten 1a3f1, 1a2j3, 1a3h1 und 1a4d1 von Fußgefäßen, 1b2a2, 1b2a7, 1b3d1 und 1b4f1 von Bechern/Schultergefäßen, 1e2b2, 1e3a3 und 1e4d1 von Näpfen, 2b2c3, 2b2e3 und 2b4a5 von Schüsseln

${ }^{92}$ Varianten $1 \mathrm{a} 2 \mathrm{e} 1$ und $1 \mathrm{a} 3 \mathrm{e} 2$ von Fußgefäßen, $1 \mathrm{~b} 4 \mathrm{i} 2$ von Bechern/Schultergefäßen, 2b2a1, 2b2e1, 2b3d1 und 2b3g2 von Schüsseln. 
Man findet 13 keramische Formvarianten (4,28 \%), die allein während der kP-4 erscheinen, ${ }^{93}$ und die Zahl der für die kP-4 und kP-5 typischen Varianten beträgt 20 (6,58 \%). ${ }^{94}$ In größter Zahl sind zugleich Formvarianten befindlich, die nur in der kP-5 bestätigt werden können; es handelt sich um nicht weniger als 70 Typen der Grab-

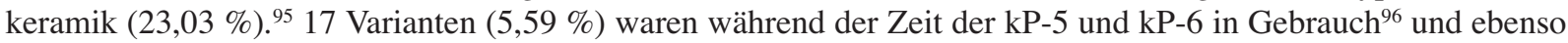
17 Typen sind nachgewiesen, die nur die kP-7 charakterisieren (Tabelle 2, Diagramm 8). ${ }^{97}$

\subsection{Vorkommen und Verteilung der Gräber nach Formvarianten (Typen)}

Ergebnisse der Hauptkoordinatenanalyse der keramischen Formvarianten (Typen) in fünf künstlichen Perioden, auf Grund von 219 Gräbern und 210 Varianten zeigt Diagramm 9. Auch in diesem Fall setzen wir voraus, dass diese künstlichen Perioden nach Formvarianten der Grabkeramik die relativchronologische Entwicklung der Lengyel-Kultur im westlichen Karpatenbecken darstellen dürften.

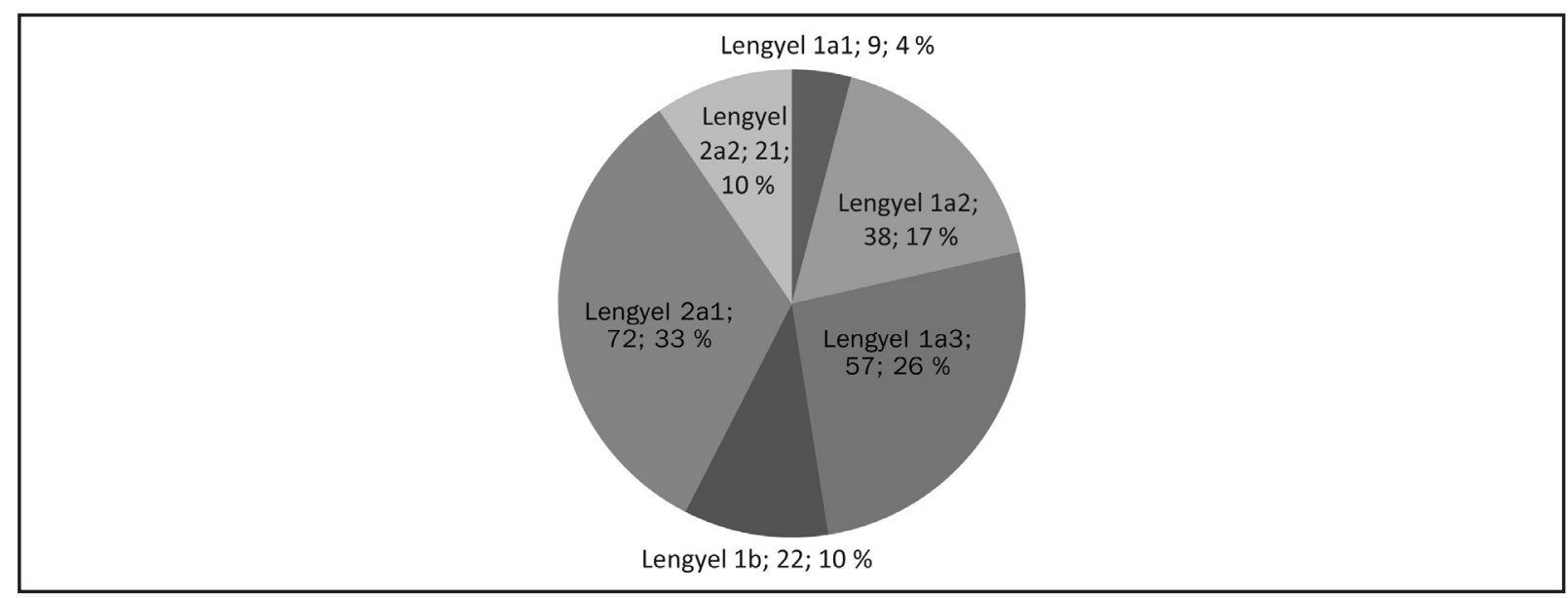

Diagramm 9. Verteilung der nach Formvarianten seriierten Gräber nach Belegungsperioden

1. Der Belegungsstufe 1 nach Formvarianten kann 21,46 \% $(n=47)$ der Gräber zugeordnet werden. Die älteste Gruppe der untersuchten Gräber erscheint auf der rechten Seite des Koordinatensystems (grün markiert). Die Bestattungen dieser Phase werden innerhalb eines aus Stufen, Phasen und Perioden bestehenden Systems als Phase Lengyel 1a definiert. Gestützt auf die ausführlichen nachprüfbaren Analysen ist es feststellbar, dass diese Gräbereinheit in zwei Teile zu trennen ist (Diagramm 9).

4,11\% ( $\mathrm{n}=9)$ dürfte die Periode Lengyel 1a1 vertreten; es handelt sich dabei um Befunde, die ohne Ausnahme die Lužianky-Gruppe vertreten. ${ }^{98}$

${ }_{93}$ Varianten $1 \mathrm{a} 3 \mathrm{k} 1$ und $1 \mathrm{a} 3 \mathrm{k} 3$ von Fußgefäßen, $1 \mathrm{~b} 5 \mathrm{~m} 1$, 1d1b4 und 1d2a2 von Bechern/Schultergefäßen, 1e2g1 und 1e2g2 von Näpfen, 2a2a2 und $2 \mathrm{a} 3 \mathrm{c} 2$ von Schultergefäßen, 2b2f3, 2b4a2 und $2 \mathrm{~b} 4 \mathrm{a} 9$ von Schüsseln bzw. 1c2a1 von Schalen.

${ }^{94}$ Varianten 1a1a1, 1a3e3, 1a3g1 und 1a3k4 von Fußgefäßen, 1b2f2, 1b3e1, 1b3e2, 1b3e4, 1b4d1, 1b4h1, 1b4i6, 1c2b3, 1c2b4, $1 \mathrm{c} 3 \mathrm{a} 1$ und $1 \mathrm{c} 3 \mathrm{a} 5$ von Bechern/Schultergefäßen, $1 \mathrm{e} 2 \mathrm{~d} 1$ und $1 \mathrm{e} 3 \mathrm{c} 3$ von Näpfen, 2a2d3 von Butmir-Gefäßen sowie $2 \mathrm{~b} 1 \mathrm{~b} 2$ und $2 \mathrm{~b} 2 \mathrm{c} 2$ von Schüsseln.

${ }^{95}$ Varianten 1a1a3, 1a1b2, 1a2c3, 1a2j1, 1a2j2, 1a2j4, 1a2k3, 1a2k5, 1a214, 1a3b2, 1a3i1, 1a212, 1a4a1 und 1a4c3 von Fußgefäßen, 1b2c1, 1b2f3, 1b3c2, 1b3c3, 1b3c4, 1b3e3, 1b3e5, 1b4e2, 1b4e5, 1b4e6, 1b4f2, 1b4i1, 1b4i4, 1b4i5, 1b5j2, 1b5k1, 1b511, 1c2b2, $1 \mathrm{c} 2 \mathrm{~b} 5,1 \mathrm{c} 2 \mathrm{~b} 7,1 \mathrm{c} 3 \mathrm{a} 4,1 \mathrm{c} 4 \mathrm{a} 2$, 1d1a1 und 1d1b3 von Bechern/Schultergefäßen, 1e1b1, 1e2c1, 1e2c2, 1e2c4, 1e2f6 und 1e3a2 von Näpfen, 2a1b2, 2a1c1, 2a1e2, 2a1e5, 2a1e6, 2a2a3, 2a2d1, 2a2d4, 2a2e1, 2a $2 \mathrm{f} 2$ und 2a3b2 von Butmir-Gefäßen, 2b3b4, 2b3d2, 2b4a4, 2b4a6, 2b4a7 und 2b5d1 von Schüsseln bzw. 2c1c3, 2c1d3, 2c2d1, 2c2d2, $2 \mathrm{c} 2 \mathrm{~d} 5$ und $2 \mathrm{c} 2 \mathrm{f} 1$ von Schalen.

${ }^{96}$ Varianten 1a2k4 und 1a213 von Fußgefäßen, 1b1f2, 1b1f3, 1b3c1, 1b4i3, 1c1a2, 1d1b2 und 1d2a3 von Bechern/Schultergefäßen, 2a1b3, 2a1e3 und 2a2e3 von Butmir-Gefäßen, 2b1b1, 2b3a4, $2 \mathrm{~b} 3 \mathrm{~b} 2$ und $2 \mathrm{~b} 4 \mathrm{a} 8$ von Schüsseln und $2 \mathrm{c} 2 \mathrm{~d} 4$ von Schalen.

${ }^{97}$ Varianten 1a1a4, 1a2f1, 1a2k2 und 1a3d1 von FußgefäBen, 1b5i1, 1c1a1, 1c2b8, 1c4a1, 1d1b1 und 1d2a4 von Bechern/ Schultergefäßen, 1e4b2 von Näpfen, 2a1c2, 2a2a1 und 2a2e2 von Butmir-Gefäßen, 2b2e 2 und $2 \mathrm{~b} 4 \mathrm{a} 1$ von Schüsseln sowie $2 \mathrm{c} 1 \mathrm{~d} 2$ von Schalen.

${ }^{98}$ Grab Lu1.56, Lu4.42, Lu7.42, Lu4.56, Lu5.42, Lu3.42, lux2.42, lux4.42 und Lu2.42. 
Tabelle 3. Verteilung der Gräber nach Belegungsperioden in den untersuchten Nekropolen und Gräbergruppen der Lengyel-Kultur

\begin{tabular}{|c|c|c|c|c|c|c|}
\hline \multirow{2}{*}{ Gräberfelder } & \multicolumn{3}{|c|}{ Lengyel 1a } & \multirow{2}{*}{$\begin{array}{c}\text { Lengyel 1b } \\
\text { Lengyel 1b }\end{array}$} & \multicolumn{2}{|c|}{ Lengyel 2a } \\
\hline & Lengyel 1a1 & Lengyel 1a2 & Lengyel 1a3 & & Lengyel 2a1 & Lengyel $2 \mathrm{a} 2$ \\
\hline Lužianky & 9 & $\begin{array}{c}4 \\
(10,53 \%) \\
\end{array}$ & & & & \\
\hline Friebritz & & $\begin{array}{c}1 \\
(2,63 \%) \\
\end{array}$ & $\begin{array}{c}1 \\
(1,82 \%) \\
\end{array}$ & & & \\
\hline Györe & & $\begin{array}{c}3 \\
(7,89 \%) \\
\end{array}$ & $\begin{array}{c}2 \\
(3,64 \%) \\
\end{array}$ & & & \\
\hline Zengővárkony 13 & & $\begin{array}{c}1 \\
(2,63 \%)\end{array}$ & $\begin{array}{c}2 \\
(3,64 \%) \\
\end{array}$ & & $\begin{array}{c}1 \\
(1,39 \%) \\
\end{array}$ & \\
\hline Svodín & & $\begin{array}{c}28 \\
(73,64 \%) \\
\end{array}$ & $\begin{array}{c}22 \\
(40,0 \%) \\
\end{array}$ & $\begin{array}{c}1 \\
(4,55 \%) \\
\end{array}$ & & \\
\hline Zengővárkony $6 \mathrm{c}$ & & $\begin{array}{c}1 \\
(2,63 \%) \\
\end{array}$ & $\begin{array}{c}11 \\
(20,0 \%)\end{array}$ & $\begin{array}{c}\mathbf{3} \\
(13,64 \%) \\
\end{array}$ & $\begin{array}{c}\mathbf{2} \\
(2,78 \%)\end{array}$ & $\begin{array}{c}1 \\
(4,76 \%)\end{array}$ \\
\hline Zengővárkony 2a & & & $\begin{array}{c}1 \\
(1,82 \%)\end{array}$ & & & \\
\hline Pécsvárad & & & $\begin{array}{c}1 \\
(1,82 \%) \\
\end{array}$ & & & \\
\hline Szekszárd-Ágostonpuszta & & & $\begin{array}{c}1 \\
(1,82 \%) \\
\end{array}$ & & & \\
\hline Pári-Altacker & & & $\begin{array}{c}2 \\
(3,64 \%) \\
\end{array}$ & $\begin{array}{c}1 \\
(4,59 \%) \\
\end{array}$ & & \\
\hline Zengővárkony 6a & & & $\begin{array}{c}\mathbf{3} \\
(5,45 \%)\end{array}$ & $\begin{array}{c}1 \\
(4,59 \%)\end{array}$ & & \\
\hline Zengővárkony 6b & & & $\begin{array}{c}\mathbf{3} \\
(5,45 \%)\end{array}$ & $\begin{array}{c}1 \\
(4,59 \%)\end{array}$ & & \\
\hline Zengővárkony $2 b$ & & & $\begin{array}{c}1 \\
(1,81 \%)\end{array}$ & & $\begin{array}{c}\mathbf{3} \\
(4,17 \%) \\
\end{array}$ & \\
\hline Zengővárkony 9 & & & $\begin{array}{c}2 \\
(3,64 \%) \\
\end{array}$ & $\begin{array}{c}1 \\
(4,59 \%) \\
\end{array}$ & $\begin{array}{c}2 \\
(2,78 \%) \\
\end{array}$ & $\begin{array}{c}1 \\
(4,76 \%) \\
\end{array}$ \\
\hline Mórágy & & & $\begin{array}{c}\mathbf{3} \\
(5,45 \%) \\
\end{array}$ & $\begin{array}{c}\mathbf{5} \\
(22,73 \%) \\
\end{array}$ & $\begin{array}{c}21 \\
(29,17 \%) \\
\end{array}$ & $\begin{array}{c}10 \\
(47,62 \%) \\
\end{array}$ \\
\hline Zengővárkony 14a & & & & $\begin{array}{c}1 \\
(4,59 \%)\end{array}$ & & \\
\hline Zengővárkony 5 & & & & $\begin{array}{c}1 \\
(4,59 \%) \\
\end{array}$ & $\begin{array}{c}\mathbf{1} \\
(1,39 \%)\end{array}$ & \\
\hline Zengővárkony 6d & & & & $\begin{array}{c}1 \\
(4,59 \%) \\
\end{array}$ & $\begin{array}{c}4 \\
(5,56 \%) \\
\end{array}$ & \\
\hline Zengővárkony 8b & & & & $\begin{array}{c}2 \\
(9,09 \%) \\
\end{array}$ & $\begin{array}{c}1 \\
(1,39 \%) \\
\end{array}$ & \\
\hline Alsónyék 10B & & & & $\begin{array}{c}\mathbf{3} \\
(13,64 \%) \\
\end{array}$ & $\begin{array}{c}\mathbf{1 5} \\
(20,83 \%) \\
\end{array}$ & $\begin{array}{c}2 \\
(9,52 \%) \\
\end{array}$ \\
\hline Veszprém & & & & $\begin{array}{c}1 \\
(4,59 \%) \\
\end{array}$ & $\begin{array}{c}1 \\
(1,39 \%) \\
\end{array}$ & $\begin{array}{c}2 \\
(9,52 \%) \\
\end{array}$ \\
\hline Zengővárkony 7 & & & & & $\begin{array}{c}1 \\
(1,39 \%) \\
\end{array}$ & \\
\hline Villánykövesd & & & & & $\begin{array}{c}3 \\
(4,17 \%) \\
\end{array}$ & $\begin{array}{c}1 \\
(4,76 \%) \\
\end{array}$ \\
\hline Alsónyék 5603 & & & & & $\begin{array}{c}17 \\
(23,61 \%) \\
\end{array}$ & $\begin{array}{c}\mathbf{3} \\
(14,29 \%) \\
\end{array}$ \\
\hline Zengővárkony 10 & & & & & & $\begin{array}{c}1 \\
(4,76 \%) \\
\end{array}$ \\
\hline
\end{tabular}


$17,35 \%(\mathrm{n}=38)$ der Bestattungen schlossen wir in die Belegungsperiode Lengyel 1a2 nach Varianten ein. Vier solche Gräber (10,53 \%) können in der Lužianky-Gruppe, ${ }^{99} 28$ andere (73,68 \%) dagegen in Svodín nachgewiesen werden. ${ }^{100}$ Drei solche Befunde $(7,89 \%)$ stammen noch aus Györe ${ }^{101}$ und zwei aus Zengővárkony. ${ }^{102}$ Auch das Grab fr131 von Friebritz dürfte diese Belegungsperiode vertreten (Diagramm 9, Tabelle 3).

Der Belegungsperiode Lengyel 1a3 nach Varianten wurde 26,03 \% $(n=57)$ der seriierten Bestattungen zugeordnet. Aus Svodín stammen 22 (39,29 \%) solche Gräber. ${ }^{103}$ Ihre Zahl beträgt in Zengővárkony dagegen schon $24(42,86 \%)$, also beinahe die Hälfte der Befunde der erörterten Belegungsperiode. ${ }^{104}$ In Mórágy sind zu dieser Zeitperiode nur noch drei Bestattungen (5,36 \%) zu finden und in Györe bzw. Pári-Altacker sind je zwei Befunde (je 3,57 \%) belegt. ${ }^{105}$ In Friebritz, Pécsvárad und Szekszárd-Ágostonpuszta findet man je einen Befund dieser Belegungsperiode (Diagramm 9, Tabelle 3). ${ }^{106}$

Die Belegungsphase $1 \mathrm{~b}$ nach Formvarianten dürfte mit nicht mehr als 10,05\% $(\mathrm{n}=22)$ der seriierten Gräber als der typochronologische Übergang zwischen den frühen und späten Belegungsphasen der Lengyel-Gräberfelder angesehen werden (Diagramm 9). Ein Charakteristikum dieser Zeitperiode besteht darin, dass das Gräberfeld von Svodín durch nicht mehr als eine Bestattung (Grab sv109.80) vertreten wird. Mit dem größten Prozentsatz, mit $50,0 \%(\mathrm{n}=11)$, erscheinen jetzt Befunde in den Gräbergruppen 5, 6a, 6b, 6c, 6d, 8b, 9 und 14 von Zengővárkony. ${ }^{107}$ $22,73 \%(\mathrm{n}=5)$ der hier zugeordneten Bestattungen stammt aus Mórágy bzw. 13,64 \% $(\mathrm{n}=3)$ aus Alsónyék 10B. ${ }^{108}$ In Veszprém (Grab ve7) und Pári (Grab pa6) befindet sich je eine Bestattung (Diagramm 9, Tabelle 3).

Die nach den keramischen Formvarianten bestimmte Belegungsperiode Lengyel 2a1 nach Varianten wird mit einem Anteil von 32,88 \% $(n=72)$ repräsentiert. Diese Bestattungen treten mit dem größten Prozentsatz, mit 29,17 \% ( $\mathrm{n}=21)$, in Mórágy ${ }^{109}$ bzw. mit 23,61 \% $(\mathrm{n}=17)$ an der Fundstelle Alsónyék 5603 auf. ${ }^{110}$ Beachtenswert ist auch, dass je 20,83 \% ( $\mathrm{n}=15-15)$ dieser Befunde sowohl in Zengővárkony als auch in Alsónyék 10B nachgewiesen werden können. ${ }^{111}$ In Villánykövesd findet man drei $(4,17 \%)$ solche Bestattungen; in Veszprém-Jutasi út nur eine (Diagramm 9, Tabelle 3). ${ }^{12}$

Nicht mehr als 9,59\% $(\mathrm{n}=21)$ der nach Formvarianten seriierten Gräber gehört der Belegungsperiode Lengyel 2a2 zu. Beinahe die Hälfte, 47,62 \% ( $\mathrm{n}=10)$, dieser Befunde wurde im Gräberfeld von Mórágy festgestellt ${ }^{113}$ und 14,29\% ( $\left.\mathrm{n}=3-3\right)$ stammt aus Alsónyék 5603 bzw. Zengővárkony. ${ }^{114}$ Diese spätlengyelzeitlichen Bestattungen sind auch für die Gräbergruppen 10B von Alsónyék und Veszprém mit je 9,52\% $(\mathrm{n}=2-2)$ typisch, ${ }^{115}$ während nur eine Bestattung (Grab vk20) in Villánykövesd nachgewiesen werden kann (Diagramm 9, Tabelle 3).

${ }^{99}$ Grab luy 1.56, lux3.42, lux5.42 von Lužianky und mo16.42 aus Mlynárce.

${ }^{100} \mathrm{Grab}$ s143.81, s070.78, s013.73, s045.74, s140.81, $\mathrm{s} 156.81, \mathrm{~s} 014.73, \mathrm{~s} 139.81, \mathrm{~s} 176.82, \mathrm{~s} 037.74, \mathrm{~s} 024.74, \mathrm{~s} 048.76$, sv042.74, sv007.72, sv163.81, sv021.73, sv127.80, sv177.82, sv029.73, sv178.82, sv094.79, sv077.78, sv122.80, sv076.78, sv132.80, sv093.79, sv113.80, sv134.80. bergruppe 13)

${ }^{102}$ Grab zv214 (Gräbergruppe 6c) und Grab zv360 (Grä-

${ }^{103}$ Grab sv103.80, sv005.72, sv114.80, sv144.81, sv112.80, sv030.73, sv106.80, sv105.80, sv039.74, sv010.72, sv003.71, sv160.81, sv159.81, sv041.74, sv148.81, sv130.80, sv121.80, sv016.73, sv027.73, sv126.80, sv172.81, sv006.72 und sv009.72.

${ }^{104} \mathrm{Grab}$ zv017 (Gräbergruppe 2a), zv011 (Gräbergruppe 2b), zv056, zv057, zv059 (Gräbergruppe 6a), zv076, zv77, zv260 (Gräbergruppe 6b), zv173, zv174, zv177, zv178, zv179, zv180, zv181, zv184, zv187, zv188, zv205, zv206 (Gräbergruppe 6c), zv91, zv93 (Gräbergruppe 9), zv355, zv361 (Gräbergruppe 13).

${ }^{105}$ Grab m15, m66 und m109 von Mórágy, gy11 und gy13 von Györe bzw. pa2 und pa5 von Pári-Altacker.

${ }^{106}$ Grab fr130 von Friebritz, pv 8 von Pécsvárad und ap20 von Szekszárd-Ágostonpuszta.

${ }^{107}$ Grab zv44 (Gräbergruppe 5), zv64 (Gräbergruppe 6a), zv72 (Gräbergruppe 6b), zv182, zv185, zv201 (Gräbergruppe 6c), zv247 (Gräbergruppe 6d), zv265, zv271 (Gräbergruppe 8b), zv108 (Gräbergruppe 9), zv314 (Gräbergruppe 14a).

${ }^{108}$ Grab m16, m36, m51, m91 und m93 von Mórágy bzw. Grab an801, an3103 und an4027 von Alsónyék 10B.

${ }^{109} \mathrm{Grab} \mathrm{m} 2, \mathrm{~m} 3, \mathrm{~m} 7, \mathrm{~m} 8, \mathrm{~m} 9, \mathrm{~m} 10, \mathrm{~m} 17, \mathrm{~m} 27, \mathrm{~m} 34, \mathrm{~m} 37$, $\mathrm{m} 38, \mathrm{~m} 43, \mathrm{~m} 44, \mathrm{~m} 45, \mathrm{~m} 49, \mathrm{~m} 55, \mathrm{~m} 57, \mathrm{~m} 62, \mathrm{~m} 74, \mathrm{~m} 76$ und $\mathrm{m} 89$.

${ }^{110}$ Grab az263, az272, az318, az319, az333, az337, az699, az743, az1006, az1190, az1235, az1320, az1391, az1669, az1967, az2330, az3132.

${ }^{111}$ Grab zv5, zv12, zv14 (Gräbergruppe 2b), zv37 (Gräbergruppe 5), zv196, zv203 (Gräbergruppe 6c), zv232, zv234, zv238, zv252 (Gräbergruppe 6d), zv79 (Gräbergruppe 7), zv266 (Gräbergruppe 8b), zv088, zv135 (Gräbergruppe 9), zv350 (Gräbergruppe 13) von Zengővárkony bzw. Grab an0398, an769, an0791, an792, an796, an804, an811, an828, an853, an963, an1009, an3020, an3089, an4012 und an4028 von Alsónyék 10B.

${ }^{112}$ Grab vk4, vk10 und vk12 von Villánykövesd und Grab ve2 von Veszprém-Jutasi út.

${ }^{113}$ Grab m14, m26, m33, m39, m46, m53, m60, m80, m84 und m86 von Mórágy.

${ }^{114}$ Grab zv176 (Gräbergruppe 6c), zv326 (Gräbergruppe 9), zv144 (Gräbergruppe 10) von Zengővárkony bzw. Grab az190, az304 und az1191 von Alsónyék 5603.

${ }^{115}$ Grab an847 und an1473 von Alsónyék 10B bzw. Grab ve3 und ve5 von Veszprém-Jutasi út. 


\section{ZUR BELEGUNGSGESCHICHTE DER UNTERSUCHTEN LENGYEL-GRÄBERFELDER}

\subsection{Verteilung der typologischen Einheiten der Grabkeramik und der Gräber nach Zeitperioden}

Mit Hilfe der Merkmalanalyse und Seriation der Typengruppen und Formvarianten der lengyelzeitlichen Grabkeramik definierten wir also drei Belegungshorizonte (Lengyel 1a, Lengyel 1b, Lengyel 2a) und fünf Belegungsperioden hinsichtlich des Gebrauchs der lengyelzeitlichen Gräberfelder im westlichen Karpatenbecken. Die Verteilung der untersuchten Gräber konnte in der Beziehung unter den Typengruppen mit einem breiteren Charakter und den Formvarianten bei 235 Gräbern studiert werden. Von diesen Ergebnissen ausgehend kann Folgendes resümiert werden.

1. In der anfänglichen Zeitperiode (nach Typengruppe 1a1) überwiegen keramische Varianten der Belegungsperiode Lengyel 1a1 mit 67,0 \% $(n=6)$, die Varianten der Belegungsperiode Lengyel 1a2 sind aber schon mit $33,0 \%(\mathrm{n}=3)$ vorhanden.

2. In der zweiten Etappe (nach Typengruppe 1a2) sind die Gefäßtypen der Belegungsperiode 1a1 schon verschwunden, jene der Belegungsperiode Lengyel 1a2 erscheinen mit einem Prozentsatz von $46 \%(\mathrm{n}=21)$ und neue Gefäßtypen der Belegungsperiode Lengyel 1a3 mit $54 \%(n=25)$ kommen in Mehrheit auf.

3. Für die dritte Etappe (nach Typengruppe 1a3) sind Gefäßtypen der Belegungsperiode 1a3 mit $42 \%$ $(n=28)$ typisch, es treten gleichzeitig aber auch Gefäßtypen der Belegungsperiode Lengyel 1b mit $12 \%(n=8)$ und jene der Belegungsperiode Lengyel 2a1 mit $30 \%(n=30)$ auf. Beachtenswert ist, dass auch die Belegungsperiode Lengyel 2a2 zu dieser Zeit in einem Fall nachgewiesen werden kann.

4. In der vierten Etappe (nach Typengruppe 1b) sind Gefäßtypen der Belegungsperiode Lengyel 1b durch $25 \%(n=6)$ vertreten, jene der Belegungsperiode 2 al mit einem Anteil von $54 \%(n=13)$ überwiegen und die Proportion der Gräber mit den spätesten Gefäßtypen (Lengyel 2a2) macht schon $21 \%(\mathrm{n}=5)$ aus.

5. In der fünften Etappe (nach Typengruppe 2a1) sind Gefäßtypen der Belegungsperiode Lengyel 2a1 mit $30 \%(\mathrm{n}=22)$ vorhanden, aber der Anteil der Typen der Belegungsperiode Lengyel 2a2 beträgt schon $70 \%(\mathrm{n}=51)$.

6. In der letzten Etappe (nach Typengruppe 2a2) befinden sich Gräber $(n=16)$, die ausschließlich Gefäßtypen der spätesten Belegungsperiode der Lengyel-Kultur (Lengyel 2a2) aufweisen.

Von diesen Daten ausgehend kann festgestellt werden, dass die Belegung der lengyelzeitlichen Gräberfelder im westlichen Karpatenbecken in Lužianky begonnen hat. In der Lužianky-Gruppe sind Gräber der Periode Lengyel 1a1 durch einen Prozentsatz von $69 \%(n=9)$ und jene der Periode Lengyel 1a2 nur mehr durch $31 \%$ $(\mathrm{n}=31)$ vertreten. Bestattungen aus den nachfolgenden Zeitperioden sind auf diesem Fundort nicht vorhanden (Tabelle 3, Diagramm 10).

Die erste Belegung des Gräberfeldes von Svodín, mehr als die Hälfte - 54,90 \% ( $\mathrm{n}=28)$ - der untersuchten Bestattungen, kann in die Belegungsperiode Lengyel 1a2 datiert werden und die Benutzung dieser Nekropole setzte auch in der Belegungsperiode Lengyel 1a3, mit einem Anteil von 43,14 \% ( $n=22)$ der Gräber, fort. Nur ein Grab $(1,96 \%)$ dürfte darauf hinweisen, dass man sich in diesem Gräberfeld auch in der Übergangsperiode Lengyel $1 \mathrm{~b}$ bestatten ließe (Tabelle 3, Diagramm 10, Diagramm 11). In Bezug auf die Periodisierung der Lengyel-Kultur ist es notwendig zu erwähnen, dass die hier den Belegungsperioden Lengyel 1a1, Lengyel 1a2 und Lengyel 1a3 zugeordneten Gräber von Svodín von P. Demján in seine Perioden I-IV eingeschlossen wurden. ${ }^{116}$ Eine Ausnahme stellt das Grab sv109.80 dar, das nach den vorliegenden Ergebnissen unsere Übergangsperiode Lengyel 1b vertreten dürfte.

Je eine Bestattung aus Friebritz dürfte die Belegungsperioden Lengyel 1a2 und Lengyel 1a3 repräsentieren. Drei Gräber von Györe stammen aus der Periode Lengyel 1a2 und nur zwei aus der Periode Lengyel 1a3 (Tabelle 3).

Der Anfang der Belegung des Gräberfeldes von Zengővárkony wurde durch die Ergebnisse der Merkmalanalyse und Seriation in die Belegungsperiode Lengyel 1a2 datiert, im Kreis der seriierten Gräber befindet sich aber nicht mehr als 3,64 \% der Gräber $(n=2)$, die aus dieser Zeit gestammt haben dürften. Beinahe die Hälfte, 43,64 \% $(\mathrm{n}=24)$, der Befunde kann in die Belegungsperiode Lengyel 1a3 datiert werden und der Anteil der Gräber der Übergangsperiode Lengyel 1b macht nur 20,0 \% $(n=11)$ aus. Die Benutzung dieser Nekropole dauerte während der Belegungsperiode Lengyel 2a1 mit 27,27 \% $(n=15)$ der Bestattungen und endete zur Zeit der Belegungsperiode Lengyel 2a2, als der Anteil der Gräber schon nicht mehr als 5,45\% $(\mathrm{n}=3)$ beträgt (Tabelle 3, Diagramm 10, Diagramm 12).

${ }^{116}$ DEMJÁN 2010. 
Im Fall des Gräberfeldes von Zengővárkony handelt es sich zugleich um eine Fundstelle mit mehreren Gräbergruppen und -gruppierungen, die sich nicht einem ähnlichen Schema gemäß belegt hatten. Auf die Entwicklung der behandelten Gräbergruppen von Zengővárkony wird in Tabelle 3 hingewiesen: In den Gräbergruppen 13 und $6 \mathrm{c}$ wird die Belegungsperiode Lengyel 1a2 nur durch je ein Grab vertreten und die Mehrheit der Bestattungen stammt auch in den anderen Gräbergruppen aus den Perioden Lengyel 1b, Lengyel 2a1 und Lengyel 2a2. Die Gräbergruppe $6 \mathrm{c}$ zeichnet sich von den anderen dadurch aus, dass hier 11 Bestattungen zur Belegungsperiode Lengyel 1a2 gehören und die Benutzung dieser Gräbergruppe auch noch in der jüngsten Periode fortgesetzt wurde. Ein ähnliches Phänomen kann auch im Fall der Gräbergruppe 9 beobachtet werden (Tabelle 3, Diagramm 10, Diagramm 12).

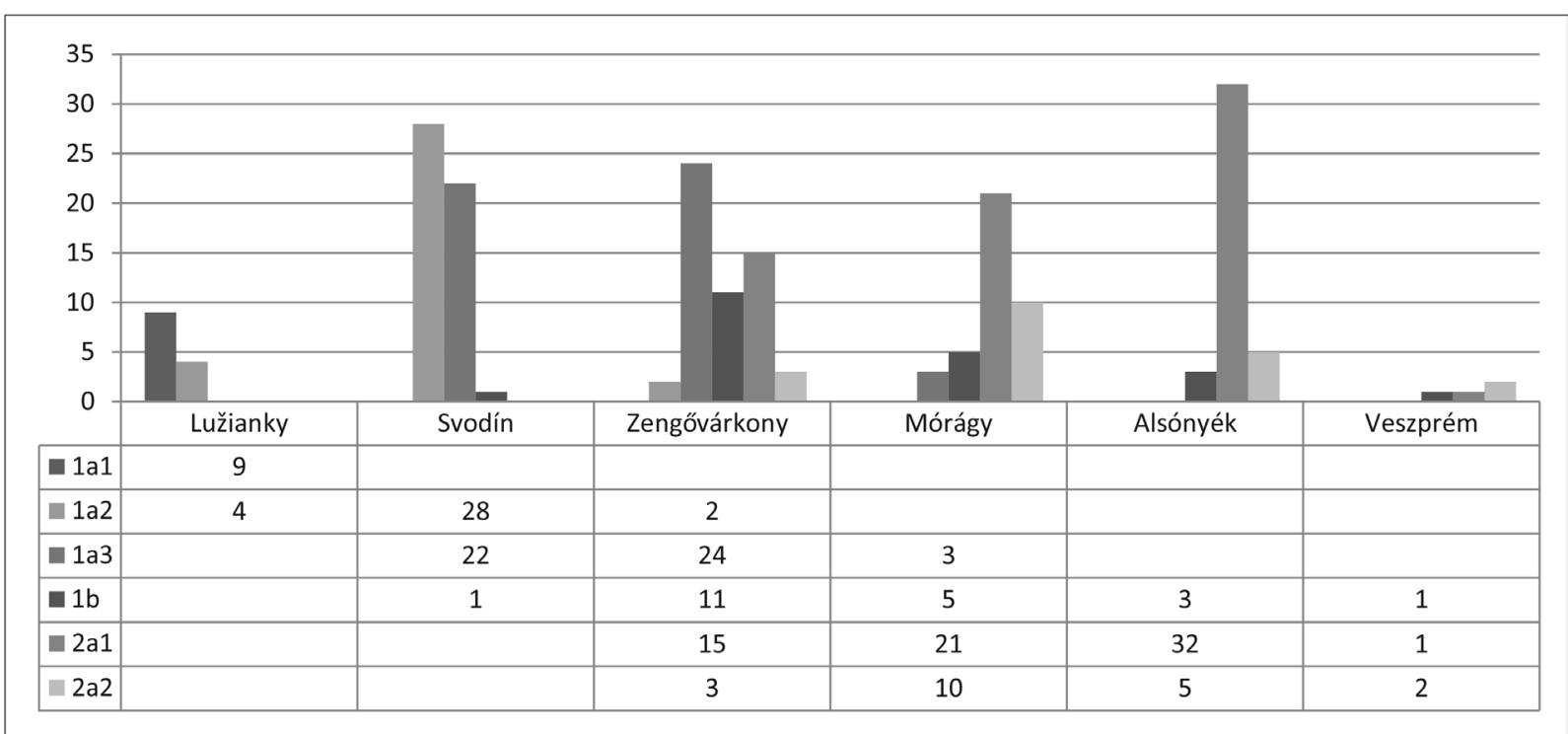

Diagramm 10. Belegungsgeschichte der größten Lengyel-Gräberfelder

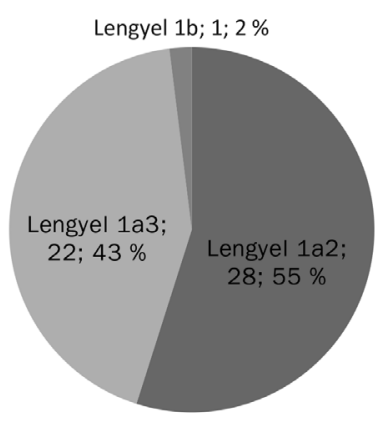

Diagramm 11. Verteilung der Gräber von Svodín nach Belegungsperioden

Die Benutzung des Gräberfeldes von Mórágy begann nur in der Belegungsperiode Lengyel 1a3, mit einem Prozentsatz von 7,69 \% ( $\mathrm{n}=3)$ der seriierten Bestattungen. Der Anteil der Gräber beträgt auch während der Übergangsperiode Lengyel $1 \mathrm{~b}$ nicht mehr als 12,82\% $(\mathrm{n}=5)$. Eindeutig überwiegen hier die Befunde der späteren Perioden, jene der Belegungsperiode Lengyel 2a1 mit mehr als der Hälfte, 53,85 \% $(\mathrm{n}=21)$, der Gräber. 25,64 \% ( $\mathrm{n}=10)$ der Bestattungen wurde dagegen in die Belegungsperiode Lengyel $2 \mathrm{a} 2$ datiert (Tabelle 3, Diagramm 10, Diagramm 13).

$7,50 \%(\mathrm{n}=3)$ der Befunde von Alsónyék stammt aus der Übergangsperiode Lengyel 1b, ältere Bestattungen wurden hier nicht geborgen. Diese Nekropole war überwiegend in der Belegungsperiode Lengyel 2a1 benutzt; solche Gräber treten hier sogar mit 80,0 \% ( $\mathrm{n}=32)$ auf. Auch die Bestattungen der jüngsten bekannten Belegungs- 


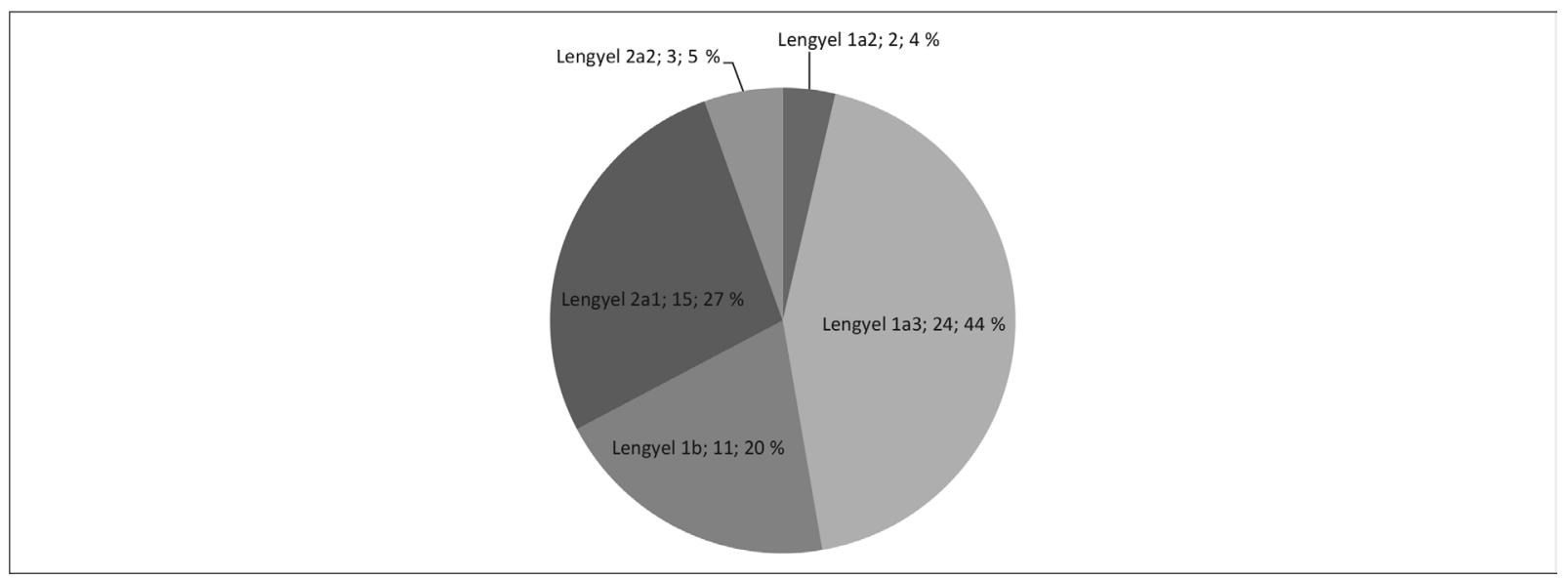

Diagramm 12. Verteilung der Gräber von Zengővárkony nach Belegungsperioden

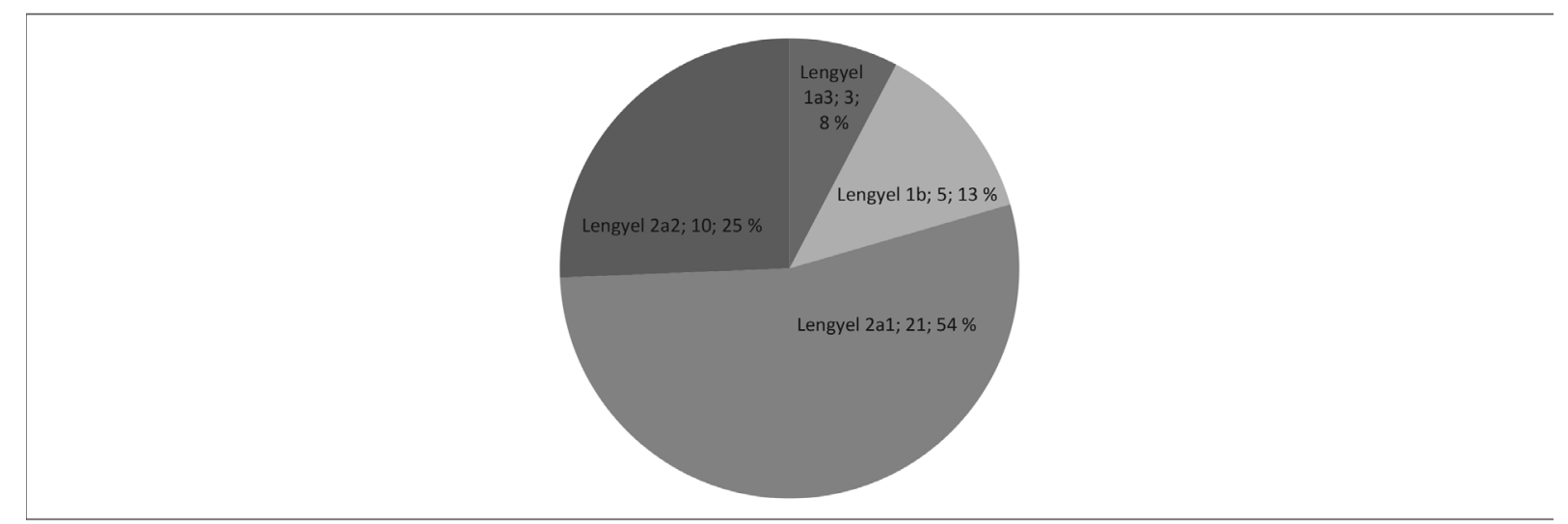

Diagramm 13. Verteilung der Gräber von Mórágy nach Belegungsperioden

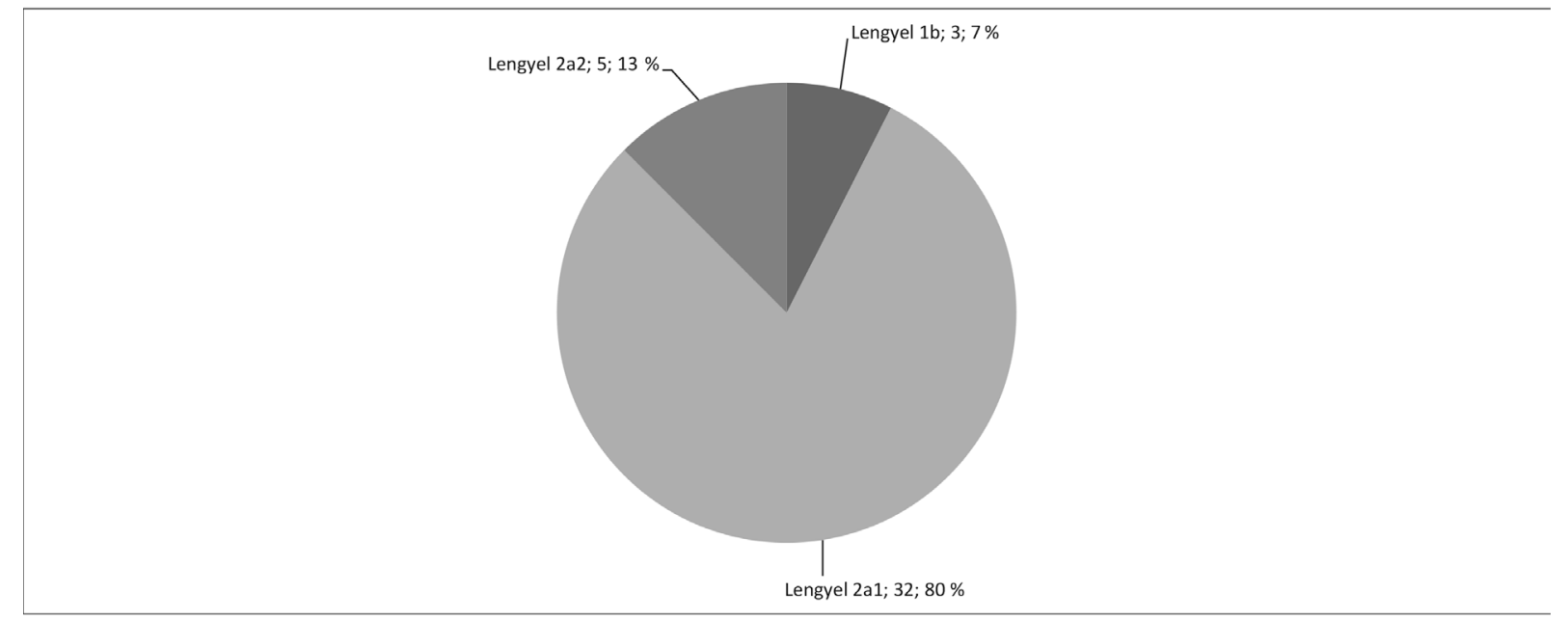

Diagramm 14. Verteilung der Gräber von Alsónyék nach Belegungsperioden 
periode des Fundortes (Lengyel 2a2) erscheinen mit einem Prozentsatz von 12,50 \% $(\mathrm{n}=5)$. Der Gebrauch der Gräbergruppe 10B von Alsónyék begann etwas früher, mit drei Bestattungen der Übergangsperiode Lengyel $1 \mathrm{~b}$. Befunde dieser Belegungsperiode wurden aus der Gräbergruppe 5603 von Alsónyék bislang nicht nachgewiesen (Tabelle 3, Diagramm 10, Diagramm 14). Nicht untersucht werden konnte der Beginn der Belegung des Gräberfeldes von Alsónyék. Den Beginn der lengyelzeitlichen Belegung betreffend kann man in Südtransdanubien dagegen anhand anderer Gräberfelder Konsequenzen ziehen.

Die Belegung der kleinen und geschlossenen Gräbergruppe von Veszprém-Jutasi út begann in der Übergangsperiode Lengyel $1 \mathrm{~b}$ und setzte sich auch in den Perioden Lengyel 2a1 und Lengyel 2a2 fort. Das Fußgefäß ve6.2 (Variante 1a2h1) aus dem Grab ve6 hat seine Entsprechung in der Sava-Gruppe der slowenischen LengyelKultur an der Fundstelle von Čatež-Sredno polje. ${ }^{117}$ Auch die Parallelfunde der Varianten 1a1a2 und 1a1a3 der südtransdanubischen Fußgefäße sind im Fundamaterial dieses Fundortes zu finden. ${ }^{18}$ Die Zusammenhänge zwischen Alsónyék und Veszprém-Jutasi út sind anhand der keramischen Typologie offensichtlich, das Grab ve9 enthielt gleichzeitig aber auch den Halsteil eines glockenförmig profilierten Hohlfußes. ${ }^{119}$ Eine Tatsache ist es weiterhin, dass das in die mittlere Kupferzeit datierte Grab ve9 von Veszprém weit nordöstlich von den Bestattungen der Lengyel-Kultur lag $^{120}$ und mit jenen in keinem zeitlichen Zusammenhang gewesen zu sein scheint.

\subsection{Beweise der vergleichenden prähistorischen Methode}

Die Ergebnisse der mit Hilfe der vergleichenden prähistorischen Methode durchgeführten Untersuchungen machen die folgenden Schlussfolgerungen im Hinblick auf die potentiellen Zusammenhänge (,,Wirkungen und/oder Gegenwirkungen“) zwischen den erörterten Fundorten und Regionen der Lengyel-Kultur im westlichen Karpatenbecken möglich.

Die möglichen typologischen Parallelen der Variante 1a1a3 der Fußgefäße (Grab an828 von Alsónyék) dürften Keramiken aus Vel'ké Kostolany (Nagykosztolány, Slowakei) darstellen. Diese Funde wurden in die Stufe Lengyel II nach J. Pavúk eingereiht. ${ }^{121}$ Im Fall der Fußgefäßvarianten 1a2k3 (Grab az699.1) und 1a2k5 (Grab az1937) sind Ähnlichkeiten oder sogar Übereinstimmungen bei den hohen Hohlfüßen bzw. der mit kurzem Hohlfuß versehenen Variante 1a1a3 (Grab az337 und an828) in Komjatice-Tomášové (Komját, Slowakei) sichtbar. ${ }^{122}$ Weitere typologische Übereinstimmungen kann man zwischen Alsónyék und Komjatice bei den Schüsselvarianten 2 b3b2 (Grab az190) und 2b3d2 (Grab az318) bzw. den Schalenvarianten 2c2e1 (Grab an795), 2c1d3 (Grab az724) und 2c2d4 (Grab az3132) entdecken. ${ }^{123}$

Auch die Funde aus Žlkovce (Zsúk, Slowakei) weisen die Merkmale der Stufe Lengyel II (Pečeňady) ,und mit denen der Phase Santovka oder MMK Ibi in Mähren und Österreich" nach J. Pavúk auf. ${ }^{124}$ Als mögliche Analogien können die Exemplare der Fußgefäßvarianten 1a1a3 (Grab an828 und az333) bzw. 1a1a4 (Grab az304) von Alsónyék behandelt werden. Im Fall der Fußgefäßvariante 1a3a1 von Alsónyék (Grab az 743) ist nur die Hohlfußform denen aus Žlkovce ähnlich. ${ }^{125}$ Auch die Bechervarianten 1b3e1 und 1c2b2 von Alsónyék (Grab an792, an4012) sind denen von Žlkovce ähnlich. ${ }^{126}$

Typologische Ähnlichkeiten sind auch zwischen der Keramik von Alsónyék und Pečeňady (Besenyőpetőfalva) besonders bei den doppelkonischen Bechern zu belegen. Bei den Becher- und Napfvarianten 1c3a4 und 1e2c3 (Grab az263 und az333) von Alsónyék handelt es sich um solche, die nur ähnlich geformt sind. ${ }^{127}$ Auch den Varianten 1b3c3 und 1b3e1 (Grab an799, an805 und an792) sowie 1c1a1 (Grab an847), 1c2b2 (Grab an811 und an4012) und 1c2b5 (Grab an811) von Alsónyék ähnliche Formen treten in Pečeňady auf. ${ }^{128}$

Die Näpfe der Varianten 1e2c3 (Grab az263) und 1e3a2 (Grab an1008 und an3020) von Alsónyék ähneln mehr oder weniger denen der Topolcsány-Szob-Übergangsphase. ${ }^{129}$
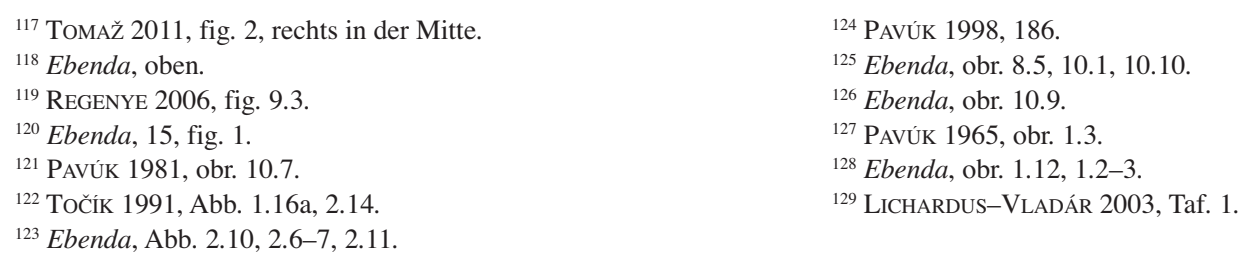
Die sog. Nyitra-Brodzany-Phase bildet die Grenze mit ihrer unbemalten Keramik zwischen der mit bemalter Keramik charakterisierten Stufe und der Ludanice-Gruppe. Hierbei handelt es sich um die Stufe Lengyel IV (Brodzany-Nitra) nach J. Lichardus, J. Vladár und A. Točík bzw. um die Stufe III (Brodzany) nach J. Pavúk. ${ }^{130}$ Im Fundmaterial dieser Phase sind Keramiken vorhanden, die sich nach ihrer Formgebung denen der Bechervarianten 1b3c3, 1b3e1 und 1b4h1 (Grab an805, an792, an4027) von Alsónyék ähneln, ${ }^{131}$ ebenso wie die Napfvarianten 1e2c1 (Grab az1967), 1e2c4 (Grab az333.4 ohne Buckel) und 1e2c3 (Grab az319). ${ }^{132}$ Die Napfvariante 1e2c3 (Grab az319) besitzt auch Henkeln und die Entsprechungen der Napfvarianten 1e3a2 (Grab an1008) und 1e3a3 (Grab an3103) sind ebenfalls an der Fundstelle von Nitra-Leningradska ulica (Nyitra, Slowakei) zu entdecken. ${ }^{133}$ Gleichzeitig ist es aber bemerkenswert, dass die in Brodzany (Brodzány, Slowakei) bekannten, glockenförmig profilierten Hohlfüße der Fußgefäße in Alsónyék fehlen. ${ }^{134}$

Zwischen Südosttransdanubien und Ostösterreich sind die annehmbaren Kontakte auf dem Niveau der keramischen Typologie während der spätlengyelzeitlichen Entwicklungszeit weniger offensichtlich oder erkennbar. Der Hohlfuß eines Fußgefäßes der Bisamberg-Oberpullendorf-Gruppe aus Vösendorf steht nach Formgebung denen der 1a1a3 (Grab an4028, az333 und az337) von Alsónyék nahe, die Schüsselpartie ist dagegen anderswie modelliert. ${ }^{135}$ Dasselbe kann auch im Fall einer anderen Keramik der Epilengyel-Zeit von Siegendorf (Grab VII/1984) festgestellt werden. ${ }^{136}$

\section{ERGEBNISSE}

1. In der vorliegenden Arbeit wurde versucht, die relativchronologische Stellung des Gräberfeldes von Alsónyék und anderer Nekropolen innerhalb des weiten Verbreitungsgebietes der Lengyel-Kultur im westlichen Karpatenbecken mit Hilfe der Merkmalanalyse und Seriation der Grabkeramik festzustellen. Die ungewöhnlich große Zahl der im Gräberfeld von Alsónyék-Bátaszék geborgenen Befunde und Funde machte die erneute Analyse der Grabkeramik der Lengyel-Kultur notwendig. Es handelt sich dabei um einen Versuch, Synchronismen zwischen den verschiedenen Regionen der Lengyel-Kultur des 5. Jahrtausends auf Grund der Gräberfeldanalyse feststellen zu können. Die untersuchten lengyelzeitlichen Grabgefäße aus dem westlichen Karpatenbecken klassifizierten wir nach 8 Gattungen, 29 Serien, 146 Typengruppen und 384 Varianten (Typen).

2. Die typologischen Merkmale der Keramikgefäße zeigen innerhalb der einzelnen Typengruppen eine viel größere Ähnlichkeit zueinander als im Fall der Serien und bei den Typengruppen kann eine viel größere Streuung nach Regionen/Fundorten beobachtet werden als bei den Serien. Bei den Formvarianten (Typen) kann man eine noch viel größere Streuung nach Regionen/Fundorten erfahren als im Fall der vorigen typologischen Einheiten.

3. Die zeitliche Reihenfolge konnten wir durch die Seriation bei 116 Typengruppen und 260 Gräbern feststellen. Die Seriationsgruppen der keramischen Typengruppen dürften die relativchronologische Entwicklung der lengyelzeitlichen Grabkeramik bzw. der Gräber und Gräberfelder im westlichen Karpatenbecken gespiegelt haben. Die zeitliche Reihenfolge oder relativchronologische Entwicklung nach Varianten (Typen) der lengyelzeitlichen Grabkeramik bestimmten wir mit Hilfe der Seriation schon im Fall von 219 Gräbern und 210 Typen.

4. Die typologischen Überlappungen zwischen den behandelten Fundorten und Regionen der LengyelKultur, die wir anhand der keramischen Merkmalanalyse und Seriation voraussetzen, werfen die Frage auf, wie diese Überlappungen auch chronologisch gewertet werden könnten. Auf die möglichen Zusammenhänge zwischen den diskutierten Fundorten und Regionen wird in Diagramm 15 hingedeutet. Es ist aber notwendig in Betracht zu ziehen, dass diese Daten jedoch irgendwie von der Menge der untersuchten Funde und Befunde der einzelnen Fundorte und Regionen stark abhängig sind. Die erkennbaren Unterschiede können aber auch die Folgen der allmählichen Ausbreitung der Lengyel-Kultur im Laufe der Geschichte (Entwicklung) der Kultur (Bevölkerung) im Laufe der Zeit sein. Diese Behauptung kann größtenteils für die spätlengyelzeitliche Entwicklung (Zengővárkony, Mórágy, Alsónyék) typisch sein, als die Zeichen der Siedlungskonzentration und der deutlichen Zunahme der Be-

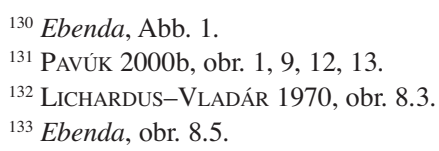

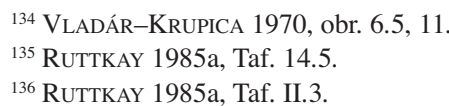

${ }^{136}$ RUTTKAY 1985a, Taf. II.3. 


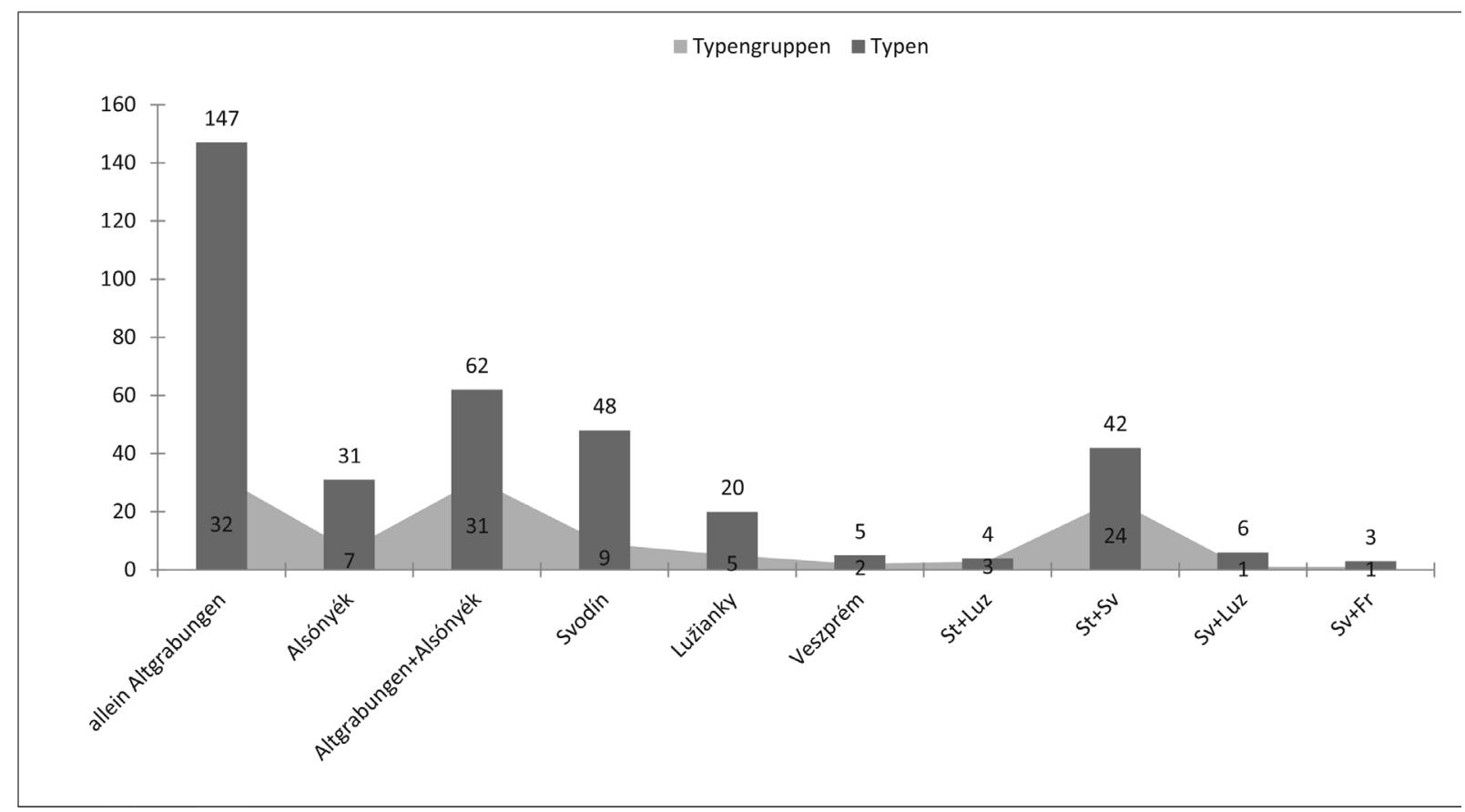

Diagramm 15. Verhältnisse zwischen Typengruppen und Typen der Grabkeramik in den untersuchten Fundstellen und Regionen der Lengyel-Kultur im westlichen Karpatenbecken $(\mathrm{St}=$ Südtransdanubien, Luz = Lužianky, Sv = Svodín, Fr = Friebritz)

völkerung im Spätneolithikum sowohl im östlichen als auch im westlichen Karpatenbecken erkennbar sind (Diagramm 15).

Auf Grund der studierten keramischen Typengruppen und Typen ist es feststellbar, dass nur noch 5 Typengruppen und 20 Typen der Grabkeramik in der Lužianky-Gruppe, also am Anfang der Entwicklung der LengyelKultur, nachweisbar sind (Diagramm 15).

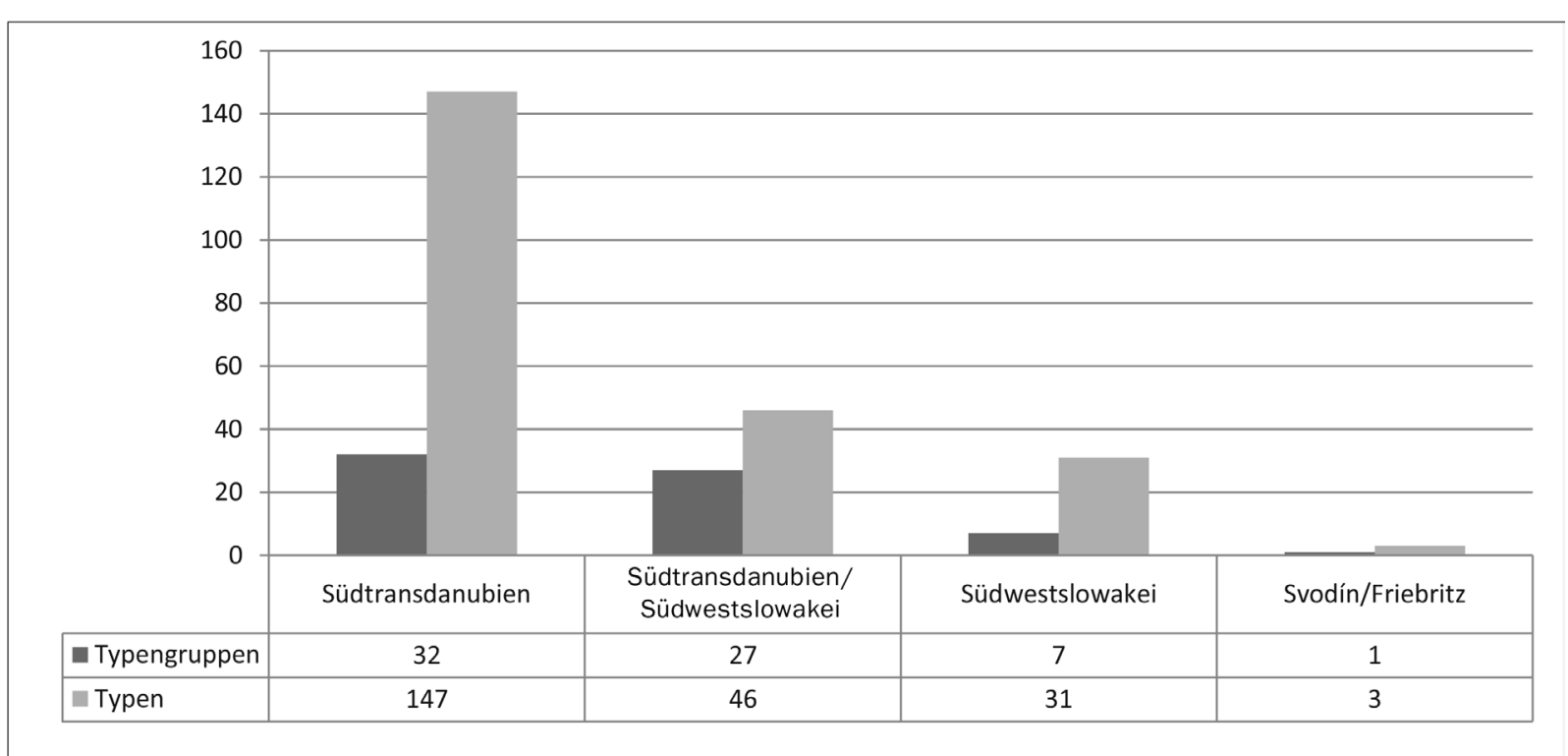

Diagramm 16. Verteilung der typochronologischen Einheiten der Grabkeramik nach Regionen der Lengyel-Kultur 
Die Menge dieser typologischen Einheiten ist im Gräberfeld von Svodín schon größer; es handelt sich bereits um 9 Typengruppen und 48 Typen. Beachtenswert ist das Phänomen zur gleichen Zeit, dass nur eine gemeinsame Typengruppe und sechs gemeinsame Typen der Grabkeramik auf die möglichen Zusammenhänge zwischen Lužianky und Svodín hindeuten. Im Fall der Nekropolen von Svodín und Friebritz kann man nur eine gemeinsame Typengruppe und nur drei Typen ausweisen: Diesmal sind aber beträchtliche Unterschiede in der Menge der untersuchten Befunde zu sehen (Diagramm 15).

Auf die Kontakte zwischen Südtransdanubien und Lužianky, die in der keramischen Typologie angenommen werden können, weisen drei Typengruppen mit vier Typen der Grabkeramik hin. Wahrscheinlich ist, dass diese Kontakte zwischen Südtransdanubien und Svodín viel kräftiger waren, wodurch sogar 24 gemeinsame Typengruppen und 42 Typen belegt werden können (Diagramm 15). Die in denjenigen bis jetzt verzeichneten Daten aus Sicht der Zusammenhänge zwischen Fundorten und Regionen können in erster Linie für die Perioden der Stufe Lengyel 1 für gültig gehalten werden (Diagramm 15).

Man findet 32 Typengruppen und 147 Typen der Grabkeramik, die allein im Fundbestand der südtransdanubischen Altgrabungen (ohne Alsónyék) auftreten. Sie umfassen alle Entwicklungsperioden der Lengyel-Kultur. Selbst im Gräberfeld von Alsónyék trifft man nicht mehr als 7 Typengruppen und 31 Typen, die nur für diesen Fundort charakteristisch sind. Es sind schließlich sogar 31 Typengruppen und 62 Typen, die sowohl in den Gräbern der südtransdanubischen Altgrabungen als auch in denen von Alsónyék befindlich sind und die in den Bestattungen anderer Fundorte der Lengyel-Kultur jedoch nicht nachgewiesen werden können. In diesem Fall hat man mit den Funden und Befunden der spätlengyelzeitlichen Entwicklung zu tun (Diagramm 15).

Tabelle 4. Radiokarbondaten der Fundorte der Lengyel-Kultur im westlichen Transdanubien

\begin{tabular}{|c|c|c|c|c|}
\hline Fundort & Periode & \multicolumn{2}{|c|}{ cal BC } & Literatur \\
\hline & & $68,2 \%$ & $95,4 \%$ & \\
\hline Letenye-Szentkeresztdomb & $\begin{array}{l}\text { Spätlengyel } \\
(\text { Topolcsány-Szob = III/IV) }\end{array}$ & $\begin{array}{l}4451-4228 \\
4200-4170\end{array}$ & $4541-4037$ & Radon \\
\hline Zalavár-Mekenye & $\begin{array}{l}\text { Spätlengyel } \\
(\text { Topolcsány-Szob = III/IV) }\end{array}$ & $\begin{array}{l}4342-4226 \\
4203-4166 \\
4128-4116 \\
4098-4075\end{array}$ & $\begin{array}{l}4440-4425 \\
4370-4040 \\
4014-4001\end{array}$ & Radon \\
\hline
\end{tabular}

Tabelle 5. Absolute Chronologie der MOG durch Sequenzierung der verschiedenen Phasen, mit aufeinander folgenden Phasen (nach STADLER et al. 2006, Tab. 5)

\begin{tabular}{|c|c|c|c|c|}
\hline Phasen der MOG & Anfang cal BC & Ende cal BC & Dauer (Mw) & Generationen \\
\hline $\begin{array}{l}\text { Formativ = } \\
\mathrm{Ia} 0\end{array}$ & $4845-4755$ & $4715-4660$ & 113 & 4 \\
\hline Ia & $4715-4660$ & $4650-4580$ & 73 & 2 \\
\hline $\mathrm{Ib}$ & $4650-4580$ & $4550-4495$ & 93 & 3 \\
\hline IIa & $4550-4495$ & $4405-4345$ & 148 & 5 \\
\hline $\mathrm{IIb}$ & $4405-4345$ & $4180-4050$ & 260 & 9 \\
\hline Epi-Lengyel & $4180-4050$ & 4130-3920 & 90 & 3 \\
\hline \multicolumn{3}{|l|}{ Insgesamt: } & 777 & 26 \\
\hline
\end{tabular}

5. Gestützt auf die hier vorgestellten Ergebnisse kann mit Recht vorausgesetzt werden, dass die Entwicklung der Lengyel-Kultur in ihrem ganzen Verbreitungsgebiet in mehreren Etappen und Schritten belegt werden kann. Provisorisch wird die Frage offen gelassen, wie die einzelnen Stufen, Phasen oder Perioden der LengyelKultur einander rechtzeitig verbinden, obwohl die typologische Substanz der Keramik der einzelnen Phasen mehr oder weniger ähnlich ist (Diagramm 16).

6. Direkt für die südtransdanubische Lengyel-Kultur besitzen wir noch keine Radiokarbondaten, mit Ausnahme von Szemely-Hegyes: Anhand der Proben wird eine Zeitperiode von 4950-4790/4720-4530 cal BC für die Benutzung der frühlengyelzeitlichen Kreisgrabenanlage bestimmt. ${ }^{137}$ Die Radiokarbondaten von westtransdanubi-

${ }^{137}$ BERTÓK-GÁTI 2011. 
schem Zalaszentbalázs-Szőlőhegyi mező (Spätlengyel) datieren die Besiedlung des Fundortes in die Zeitperiode 4596-4449 cal BC. ${ }^{138}$ Weitere Radiokarbondaten wurden auch von anderen spätlengyelzeitlichen Fundorten aus Westtransdanubien veröffentlicht (Tabelle 4). ${ }^{139}$

Eine Reihe von Radiokarbondaten aus der gesamten Entwicklungszeit der Lengyel-Kultur wird in Österreich auf eine Bestimmung basiert. P. Stadler hat nicht nur die cal BC-Daten, sondern auch die Dauer der einzelnen Phasen der mährisch-ostösterreichischen Gruppe und die Zahl der Generationen pro Phasen bestimmt (Tabelle 5). ${ }^{140}$

7. Gemäß diesen Daten darf man mit Recht einen etwa 800 Jahre langen Zeitraum für die Entwicklung der MOG voraussetzen und man rechnet mit 26 Generationen in dieser Zeit. Ähnliches kann man auch für das Leben der lengyelzeitlichen Gemeinschaften in der Südslowakei und in Südtransdanubien postulieren. Diese relativ- und absolutchronologischen Verhältnisse können im westlichen Karpatenbecken - von Lužianky über Svodín, Zengővárkony und Mórágy bis Alsónyék - nur auf Grund der Ergebnisse der jetzt durchgeführten RadiokarbonUntersuchungen und der Bayes'schen Analyse ausführlich und eingehend analysiert werden.

\section{LITERATUR}

BERTÓK-GÁTI 2011

BoGNÁR-KuTZIÁN 1966a

BOGNÁR-KUTZIÁN 1966b

DEMJÁN 2010

DOHRN-IHMIG 1974

DONEUS 2001

GALLINA et al. 2010

GELENCSÉR 2010

GLESER 1995

GLESER 2012

HERTELENDI 1995

KALICZ 1969

KALICZ 1975-1976

KALICZ 1977

KALICZ 1983-1984

KALICZ 1986

KALICZ 1988
= G. BERTóK-Cs. GÁtı: Neue Angaben zur spätneolithischen Siedlungsstruktur in Südosttransdanubien. ActaArchHung 62 (2011) 1-28.

= I. BognÁR-KutZiÁn: A contribution to the chronology of the Lengyel culture. SbNM 20 (1966) 63-70.

= I. BognÁr-KutZián: Das Neolithikum in Ungarn. ArchA 40 (1966) 249-280.

= P. DEMJÁN: Hroby na sídlisku lengyelskej kultúry vo Svodíne [Exceptional Graves of the Lengyel Culture from Svodín]. Manuskript. Bratislava 2010. http://www.academia.edu/2437858/Vynimocne_hroby_lengyelskej_kultury_zo_Svodina_Exceptional_Graves_of_the_Lengyel_Culture_ from_Svodin

= M. DoHRN-IhMIG: Untersuchungen zur Bandkeramik im Rheinland. Rheinische Ausgrabungen 15 (1974) 51-142.

= M. Doneus: Die Keramik der mittelneolithischen Kreisgrabenanlage von Kamegg, Niederösterreich. MPK 46. Wien 2001.

= Zs. Gallina-P. Hornok-T. Paluch-K. Somogyi: Előzetes jelentés az M6 autópálya 10/b és 11 . számú lelőhelyrészén végzett feltárásról. Alsónyék-Bátaszék (Tolna megye), 2006-2009 (Vorbericht über die präventive Ausgrabung am Fundortsteil Nr. M6 AP To 10/B und 11 Alsónyék-Bátaszék, Komitat Tolna). WMMÉ 32 (2010), 7-100.

= Á. Gelecsér: Alsónyék/Bátaszék-Malomréti-dülő (Tolna megye, To 11. lelöhely). In: J. Kvassay (ed.): Évkönyv és jelentés a Kulturális Örökségvédelmi Szakszolgálat 2008. évi feltárásairól (Field Service for Cultural Heritage 2008. Yearbook and Review of Archaeological Investigations, Kulturális Örökségvédelmi Szolgálat/Field Service for Cultural Heritage). Budapest 2010, 16-17.

= R. GLESER: Die Epi-Rössener Gruppen in Südwestdeutschland. Untersuchungen zur Chronologie, stilistischen Entwicklung und kulturellen Einordnung. Saarbrücker Beiträge zur Altertumskunde 61. Bonn 1995.

$=$ R. GLESER: Zeitskalen, stilistische Tendenzen und Regionalität des 5. Jahrtausends in den Altsiedellandschaften zwischen Mosel und Morava. In: R. Gleser-V. Becker (Hrsg.): Mitteleuropa im 5. Jahrtausend vor Christus. Beiträge zur Internationalen Konferenz in Münster 2010. Berlin 2012, 35-103.

= E. Hertelendi: 14 Carbon dating of Zalaszentbalázs-Szőlőhegyi mező 1992-1993. Antaeus 22 (1996) 105-107.

= N. KALICZ: Einige Probleme der Lengyel-Kultur in Ungarn. ŠtZ 17 (1969) 177-205.

= N. KALICZ: Neue Forschungen bezüglich der Lengyel-Kultur in Ungarn. SPFFBU E 20-21 (19751976) 51-61.

= N. KALICZ: Früh- und spätneolithische Funde in der Gemarkung des Ortes Lánycsók (Vorbericht). JPMÉ 22 (1977 [1978]) 137-156.

= N. KALICZ: Übersicht über den Forschungsstand der Entwicklung der Lengyel-Kultur und die ältesten „Wehranlagen“ in Ungarn. Mitt. Österr. Arbeitsgemeinschaft Ur- und Frühgeschichte. MUAG 33-34 (1983-1984) 271-293.

= N. KALICZ: Über das spätneolithische Siedlungswesen in Ungarn. BÁMÉ 13 (1986) 127-138.

= N. KALICZ: Beiträge zur Entstehungsphase der Lengyel-Kultur. SlA 36 (1988) 105-118.
${ }^{138}$ Weitere Daten von hier von E. Hertelendi: 4450-4370 cal BC, 4470-4266 cal BC: HERTELENDI 1995, 105-106.
${ }^{139}$ Radon - HiNZ et al. 2012.
${ }^{140}$ STADLER et al. 2006 , Tab. 5. 
KALICZ 1991

KALICZ-KREITER-TOKAI 2007

LICHARDUS 1974

LICHARDUS-ŠIŠKA 1970

LICHARDUS-VLADÁR 1970

LICHARDUS-VLADÁR 2003

NeUgebauer-MARESCH et al. 2002

NovotNÝ 1962

OROss et al. 2011

OsZTÁs et al. 2012

PAVÚK 1965

PAVÚK 1969

PAVúK 1981

PAVÚK 1998

PAVúK 2000a

PAVúK 2000b

RACZKY 1974

Radon

REGENYE 2000

REGENYE 2006

REGENYE 2007

REGENYE 2013

RUTTKAY 1985a

RUTTKAY 1985b

RutTKAY-TESCHLER-NICOLA 1985
= N. Kalicz: Beiträge zur Kenntnis der Kupferzeit im ungarischen Transdanubien. In: J. Lichardus (Hrsg.): Die Kupferzeit als historische Epoche I-II. Symposium Saarbrücken und Otzenhausen 6.-13. 11. 1988. Saarbrücker Beiträge zur Altertumskunde 55. Bonn 1991, 347-387.

= N. KalicZ-E. KreIter-Z. M. ToKaI: Die Rolle der Sopot-Kultur in der Entstehung der LengyelKultur auf Grund der neuen Ausgrabungen in Südwestungarn (Petrivente, Becsehely) (The role of the Sopot Culture in the origin of the Lengyel Culture on the ground of the new excavations in southwestern Hungary - Petrivente, Becsehely). In: J. Kozłowski-P. Raczky (eds): The Lengyel, Polgár and Related Cultures in the Middle/Late Neolithic in Central Europe. Kraków 2007, $29-47$.

= J. LichARDUs: Die Bedeutung der Lengyel-Kultur für das frühe Äneolithikum in Mittel- und Süddeutschland. BVbl 39 (1974) 29-54.

= J. LiCHARDUS-S. ŠIŠKA: Záchranny výskum pohrebiska a sídliška lengyelskej kultúry vo Svodíne roku 1965 (Rettungsgrabung auf Gräberfeld und Siedlung der Lengyel-Kultur in Svodín im Jahre 1965). S1A 18 (1970) 311-352.

= J. LICHARDUS-J. VLADÁR: Neskorolengyelské sidliškové a hrobové nálezy z Nitri [Spätlengyelzeitliche Siedlungs- und Grabfunde aus Nitra]. S1A 18 (1970) 373-419.

= J. LichaRduS-J. VladÁR: Gliederung der Lengyel-Kultur in der Slowakei. Ein Rückblick nach vierzig Jahren. S1A 51/2 (2003) 195-216.

= Ch. Neugebauer-Maresch-J. W. Neugebauer-K. Groszschmidt-U. RandL-R. Seemann: Die Gräbergruppe vom Beginn der Bemaltkeramik im Zentrum der Kreisgrabenanlage Friebritz-Süd, Niederösterreich. PreAlp 37 (2001). Trento 2002, 187-253.

= B. NovotNÝ: Lužianska skupina a počiatky mal'ovanej keramiky na Slovensku (The Lužianky group and the beginnings of painted pottery in Slovakia). Bratislava 1962.

= K. Oross-T. Marton-A. Whittle-R. E. M. Hedges-L. J. E. Cramp: Die Siedlung der BalatonLasinja-Kultur in Balatonszárszó-Kis-erdei-dülő. In: P. Kalábková-B. Kovár-P. Pavúk-J. Šuteková (eds): PANTA RHEI. Studies in Chronology and Cultural Development of the South-Eastern and Central Europe in Earlier Prehistory. Presented to Juraj Pavúk on the Occasion of his 75. Birthday. Studia archaeologica et mediaevalia 11. Bratislava 2011, 381-407.

= A. OsZTÁs-I. ZALAI-GAÁL-E. BÁNFFY: Alsónyék-Bátaszék: A new chapter in the research of Lengyel culture. Documenta Praehistorica 39 (2012) 1-21.

= J. PAvúK: Nové nálezy lengyelskej kultúry za Slovenska (Neue Funde der Lengyel-Kultur in der Slowakei). SlA 13 (1965) 27-50.

= J. PAvÚK: Anteil des Želiezovce-Typus an der Genesis der Lengyel-Kultur. ŠtZ 17 (1969) 345-360.

= J. PAVÚK: Sučasný stav štúdia lengyelskej kultúry na Slovensku (The present state of knowledge of the Lengyel culture in Slovakia). PA 72 (1981) 255-299.

= J. PAvúK: Hlavné výsledky výskumu sídliška lengyelskej kultúry v Žlkovciach (Die Position der Moravany-Phase in der Entwicklung der Lengyel-Kultur). SlA 46 (1998) 169-186.

= J. PAVÚK: Postavenie fázy Moravany vo vývoji lengyelskej kultúry. In: Otázky neolitu a eneolitu našich zemi - 2000. Plzeň 2000, 151-160.

= J. PAVúK: Skupina Brodzany-Nitra alebo skupina Brodzany? (Gruppe Brodzany-Nitra oder Brodzany-Gruppe?). PA - Supplementum 13. Praha 2000, 328-340.

= P. RACZKY: A lengyeli kultúra legkésőbbi szakaszának leletei a Dunántúlon [Funde der spätesten Phase der Lengyel-Kultur in Transdanubien]. ArchÉrt 101 (1974) 185-210.

= M. HinZ-M. Furholt-J. MülleR-D. RAETZEl-FABIAN-CH. RinNE-K.-G. SJÖGREN-H.-P. WotzKA: Radon - Radiocarbon dates online 2012. Central European database of 14C dates for the Neolithic and Early Bronze Age. www.jungsteinsite.de, 2012, 1-4.

= J. REGENYE: A lengyeli kultúra újabb lelőhelyei Veszprém megyében (New sites of the Lengyel Culture in Veszprém County). VMMK 21 (2000) 9-19.

= J. REGENYE: Temetkezések Veszprém, Jutasi út lelőhelyen (lengyeli kultúra, Balaton-Lasinja kultúra) (Burials at the site of Veszprém, Jutasi Street). VMMK 24 (2006) 7-36.

= J. REGENYE: The late Lengyel culture in Hungary as reflected by the excavation at Veszprém. In: J. K. Kozłowski-P. Raczky (eds): The Lengyel, Polgár and Related Cultures in the Middle/Late Neolithic in Central Europe. Kraków 2007, 381-396.

= J. RegENYE: Surviving Neolithic - The Early Copper Age in Transdanubia, North of Lake Balaton. In: A. Anders-G. Kulcsár (eds): Moments in Time. Papers Presented to Pál Raczky on his $60^{\text {th }}$ Birthday. Budapest 2013, 557-568.

= E. RutTKay: Das Neolithikum in Niederösterreich. Wien 1985.

= E. RUTTKAY: Epilengyel-Siedlungsfunde und Brandgrab aus Siegendorf. In: Festschrift für Alois J. Ohrenberger. Wissenschaftliche Arbeiten aus dem Burgenland 71. Eisenstadt 1985, 13-25.

= E. RutTKAY-M. TeschleR-NicOla: Zwei Lengyel-Gräber aus Niederösterreich. ANhM 87/A (1985) 211-235. 
SEBŌK 2012

ŠIŠKA 1969

STADLER et al. 2006

STADLER-RUTTKAY 2006

STEHLI-ZIMMERMANN 1980

STROBEL 1996

TočíK 1991

TOČÍK-LICHARDUS 1966

TOMAŽ 2011

VLADÁR-KRUPICA 1970

ZALAI-GAÁL 1999

ZALAI-GAÁL 2001

ZALAI-GAÁL 2002

ZALAI-GAÁL 2003

ZALAI-GAÁL 2007a

ZALAI-GAÁL 2007b

ZALAI-GAÁL 2008a

ZALAI-GAÁL 2008b

ZALAI-GAÁL 2014

ZALAI-GAÁL et al. 2014

ZALAI-GAÁL-KÖHLER-OSZTÁS 2010

ZALAI-GAÁL-ÓDOR 2008

ZALAI-GAÁL-OsZTÁS 2009a
= K. SEBŌK: Lengyeli eredetű hatások az Alföld kései neolitikumában. Pusztataksony-Ledence 1. feltárásának első eredményei (Influences of Lengyel origin in the Late Neolithic of the Great Hungarian Plain. First results from the excavations at Pusztataskony-Ledence 1, Jász-Nagykun-Szolnok County). ArchÉrt 137 (2012) 97-123.

= S. ŠIŠKA: Zur Problematik der spätneolithischen Kulturgruppen mit bemalter Keramik in der Ostslowakei. ŠtZ 17 (1969) 415-428.

= P. Stadler-E. Ruttkay-M. Doneus-H. Friesinger-E. Lauermann-W. Kutschera-I. MateiciuCOVÁ-W. Neubauer-Ch. Neugebauer-Maresch-G. Trnka-F. Weninger-E. M. Wild: Absolutchronologie der Mährisch-Ostösterreichischen Gruppe (MOG) der bemalten Keramik aufgrund von neuen 14C-Datierungen. In: Archäologie Österreichs 16/17 (2005/2006), Sonderausgabe 53-67.

= P. STADLER-E. RuTTKAY: 14C (AMS)-datierte Fundkomplexe der MOG in Österreich. Eine typologische Anordnung des Materials nach Phasen. http://www.researchgate.net/publication/210281791 _14C\%28AMS\%29-datierte_Fundkomplexe_der_MOG_in_sterreich_Eine_typologische_Anordnung_des_Materials_nach_Phasen

= P. Stehli-A. Zimmermann: Zur Analyse neolithischer Gefäßformen. Archaeo-Physika 7 (1980) 147-177.

= M. STROBEL: Ein Beitrag zur Gliederung der östlichen Linearbandkeramik. Versuch einer Merkmalanalyse. In: SASTUMA 4-5 (1995-1996) 9-98.

= A. Tос̌íK: Erforschungsstand der Lengyel-Kultur in der Slowakei. Rückblick und Ausblick. In: J. Lichardus (Hrsg.): Die Kupferzeit als historische Epoche I-II. Symposium Saarbrücken und Otzenhausen 6.-13. 11. 1988. Saarbrücker Beiträge zur Altertumskunde 55. Bonn 1991, 301-317.

= A. TočíK-J. Lichardus: Staršia fáza slovensko-moravskej mal'ovanej keramiky na juhozápadnom Slovensku (Ältere Phase der slowakisch-mährischen bemalten Keramik in der Südwestslowakei). PA 57 (1966) 5-90.

= A. TomAŽ: The Neolithic in continental Slovenia according to the radiocarbon chronology: Where can it be placed? Geologia, Paleontologia, Paletnologia (Udine) 32 (2010) 71-86.

= J. VLADÁR-O. KRUPICA: Neskorolengyelská keramika z Brodzian (Spätlengyel-Keramik aus Brodzian). SIA 18 (1970) 353-371.

= I. ZALAI-GAÁL: A lengyeli kultúra sírjai Pári-Altackerben (Torma István ásatása 1968-ban) [Gräber der Lengyel-Kultur in Pári-Altacker (Grabung von István Torma im Jahre 1968)]. WMMÉ 21 (1999) 1-31.

= I. ZALAI-GAÁL: Die Gräbergruppe B2 von Mórágy-Tüzkődomb und der frühere Abschnitt der Lengyel-Kultur. ActaArchHung 52 (2001) 1-48.

= I. ZALAI-GAÁL: Die neolithische Gräbergruppe B1 von Mórágy-Tűzkődomb. I. Die archäologischen Funde und Befunde. Szekszárd-Saarbrücken 2002.

= I. ZALAI-GAÁL: Das Henkelgefäß aus Györe. Ein Beitrag zu den chronologischen und kulturellen Beziehungen der Lengyel-Kultur. In: E. Jerem-P. Raczky (Hrsg.): Morgenrot der Kulturen. Frühe Etappen der Menschheitsgeschichte in Mittel- und Südosteuropa. Festschrift für Nándor Kalicz zum 75. Geburtstag. Archaeolingua 15. Budapest 2003, 285-309.

= I. ZALAI-GAÁL: Von Lengyel bis Mórágy. Die spätneolithische Grabkeramik in Südtransdanubien aus den alten Ausgrabungen. I. Analyse. WMMÉ 29 (2007) 1-162.

= I. ZALAI-GAÁL: Zengővárkony - Svodín - Friebritz: Zu den chronologischen Beziehungen zwischen den territorialen Gruppen der Lengyel-Kultur aufgrund der Gräberfeldanalyse. In: J. K. Kozłowski-P. Raczky (eds): The Lengyel, Polgár and Related Cultures in the Middle/Late Neolithic in Central Europe. Kraków 2007, 147-184.

= I. ZALAI-GAÁL: An der Wende vom Neolithikum zur Kupferzeit in Transdanubien (Ungarn): Die „Häuptlingsgräber“ der Lengyel-Kultur in Alsónyék-Kanizsa-dülő. Das Altertum (Oldenburg) 53 (2008) 1-40.

= I. ZALAI-GAÁL: Von Lengyel bis Mórágy. Die spätneolithische Grabkeramik in Südtransdanubien aus den alten Ausgrabungen. II: Katalog. WMMÉ 30 (2008) 7-216.

= I. ZALAI-GAÁL: Die Frühkupferzeit im östlichen Karpatenbecken. 1. Die relative Chronologie der Gräberfelder. [Manuskript.] Budapest 2014.

= I. ZALAI-GAÁL-A. GRISSE-A. OSZTÁS-K. KÖHLER: Die durchlochten Steingeräte des südtransdanubischen Neolithikums (5. Jahrtausend v. Chr.). Archaeolingua. Budapest 2014 (im Druck).

= I. ZALAI-GAÁL-K. KÖHLER-A. OsZTÁs: Zur Typologie und Stellung von Kulttischchen der LengyelKultur im mittel- und südosteuropäischen Neolithikum. ActaArchHung 61 (2010) 307-385.

= I. ZALAI-GAÁL-J. G. ÓDOR: Early Lengyel burials at Györe in southern Transdanubia. Antaeus 29-30 (2008) 535-576.

= I. ZALAI-GAÁL-A. OsZTÁs: Neue Aspekte zur Erforschung des Neolithikums in Ungarn. Ein Fragenkatalog zu Siedlung und Gräberfeld der Lengyel-Kultur von Alsónyék, Südtransdanubien. In: 
ZALAI-GAÁL-OsZTÁs 2009b

V. Becker-M. Thomas-A. Wolf-Schuler (Hrsg.): Zeiten - Kulturen - Systeme. Gedenkschrift für Jan Lichardus. ZAKS 17. Langenweißbach 2009, 111-139.

= I. ZALAI-GAÁL-A. OsZTÁs: A lengyeli kultúra települése és temetője Alsónyék-Kanizsa-dülőben (A settlement and cemetery of the Lengyel culture at Alsónyék-Kanizsa-dűlö). In: L. Bende-G. Lörinczy (szerk.): Medinától Etéig [Von Medina bis Ete]. Szentes 2009, 245-254. 